ANNALES

POLONICI MATHEMATICI

$89.3(2006)$

\title{
The BIC of a singular foliation defined by an abelian group of isometries
}

\author{
by Martintxo Saralegi-Aranguren (Lens) \\ and RoBERT WOLAK (Kraków)
}

\begin{abstract}
We study the cohomology properties of the singular foliation $\mathcal{F}$ determined by an action $\Phi: G \times M \rightarrow M$ where the abelian Lie group $G$ preserves a riemannian metric on the compact manifold $M$. More precisely, we prove that the basic intersection cohomology $\mathbb{H}_{\bar{p}}^{*}(M / \mathcal{F})$ is finite-dimensional and satisfies the Poincaré duality. This duality includes two well known situations:
\end{abstract}

- Poincaré duality for basic cohomology (the action $\Phi$ is almost free).

- Poincaré duality for intersection cohomology (the group $G$ is compact and connected).

This paper deals with an action $\Phi: G \times M \rightarrow M$ of an abelian Lie group on a compact manifold $M$ preserving a riemannian metric on it. The orbits of this action define a singular foliation $\mathcal{F}$ on $M$. Putting together the orbits of the same dimension we get a stratification $\mathrm{S}_{\mathcal{F}}$ of $M$. This structure is still very regular. The foliation $\mathcal{F}$ is in fact a conical foliation and we can define the basic intersection cohomology $\mathbb{H}_{\bar{p}}^{*}(M / \mathcal{F})$ (cf. [15]). This invariant becomes the basic cohomology $H^{*}(M / \mathcal{F})$ when the action $\Phi$ is almost free, and the intersection cohomology $\mathbb{H}_{\bar{p}}^{*}(M / G)$ when the Lie group $G$ is compact and connected (cf. [15]).

The aim of this work is to prove that this cohomology $\mathbb{H}_{\bar{p}}^{*}(M / \mathcal{F})$ is finite-dimensional (cf. Theorem 4.2.2) and satisfies the Poincaré duality (cf. Theorem 4.3.6). This result generalizes [6], which concerns the almost free case. When $G$ is compact and connected we find these results in [8].

The paper is organized as follows. In Section 1 we define conical foliations. Section 2 is devoted to the study of isometric actions and the induced

2000 Mathematics Subject Classification: 53C12, 57R30, 55N33, 58A35, 22Fxx.

Key words and phrases: conical foliation, isometric action, basic intersection cohomology.

Research of M. Saralegi-Aranguren partially supported by the UPV-EHU 127.310-E$14790 / 2002$.

Research of R. Wolak partially supported by the KBN grant 5 P03A 04020. 
foliations. For this kind of foliations we can define the basic intersection cohomology (BIC). This is done in Section 3. The main results are proved in Section 4. The definitions of the conical foliation and BIC were introduced in a preprint of M. Saralegi and R. Wolak, Basic intersection cohomology for singular riemannian foliations, which was circulated some time ago. The definitions and some basic properties of BIC appeared in print in [15] for the first time, but only those necessary for the study of conical fibrations. The present paper provides the first published account of the general theory of BIC.

In the following, $M$ is a connected, second countable, Hausdorff, $C^{\infty}$ manifold of dimension $m$ without boundary. All the maps considered are smooth unless otherwise indicated.

\section{CONICAL FOLIATIONS}

A singular foliation whose associated stratification is conical in the sense of Goresky-MacPherson (cf. [8]) will be called a conical foliation.

1.1. Singular foliations. A regular foliation of codimension $q$ on a manifold $M$ of dimension $m$ is a partition $\mathcal{F}$ of $M$ into arc-connected immersed submanifolds, called leaves, in such a way that each $x \in M$ has the following local model:

$$
\left(\mathbb{R}^{m}, \mathcal{H}\right)
$$

where leaves are defined by $\left\{x_{1}=c_{1}, \ldots, x_{q}=c_{q} \mid c_{1}, \ldots, c_{q} \in \mathbb{R}\right\}$. We say that $\left(\mathbb{R}^{m}, \mathcal{H}\right)$ is a simple foliation. Notice that the leaves have the same dimension.

A singular foliation on a manifold $M$ is a partition $\mathcal{F}$ of $M$ into arcconnected subsets, called leaves, such that for each $x \in M$ there exists a chart $(U, \varphi)$ at this point such that:

(i) $\operatorname{Im} \varphi=V \times W$ where $V \subset \mathbb{R}^{a}$ and $W \subset \mathbb{R}^{b}$ are open neighborhoods of 0 , and $a+b=m$,

(ii) $\varphi(x)=(0,0)$,

(iii) if $L$ is a leaf, then $\varphi(L \cap U)=V \times W_{L}$ where $W_{L} \subset W$ is a countable subset.

Leaves are connected immersed submanifolds whose dimension may vary. The definition of a singular foliation is due to P. Stefan [16] and H. Sussmann [17]. For a discussion of the singular Frobenius theorem and various equivalent conditions see [10,20], and [11] for a reduction of the axioms.

Classifying the points of $M$ according to the dimension of leaves one gets the stratification $\mathrm{S}_{\mathcal{F}}$ of $M$ whose elements are called strata. The foliation is regular when this stratification has just one stratum $\{M\}$. 
A smooth map $f:(M, \mathcal{F}) \rightarrow\left(M^{\prime}, \mathcal{F}^{\prime}\right)$ between singular foliated manifolds is foliated if it sends leaves of $\mathcal{F}$ into leaves of $\mathcal{F}^{\prime}$. If $f$ is an open foliated embedding then it preserves the dimension of leaves and therefore it sends strata of $M$ into strata of $M^{\prime}$. Examples, more properties and the singular version of the Frobenius theorem can be found in [1, 15-18].

1.1.1. EXAmple. Consider a sphere $\mathbb{S}^{n-1}$ of positive dimension endowed with a singular foliation $\mathcal{G}$ without 0 -dimensional leaves. Identify the disk $\mathbb{D}^{n}$ with the cone $c \mathbb{S}^{n-1}=\mathbb{S}^{n-1} \times\left[0,1\left[/ \mathbb{S}^{n-1} \times\{0\}\right.\right.$ by the map $x \mapsto[x /\|x\|,\|x\|]$ where $[u, t]$ is a general element of the cone. We shall consider on $\mathbb{D}^{n}$ the singular foliation

$$
c \mathcal{G}=\{F \times\{t\} \mid F \in \mathcal{G}, t \in] 0,1[\} \cup\{\vartheta\},
$$

where $\vartheta$ is the vertex $[u, 0]$ of the cone. The induced stratification is

$$
\mathrm{S}_{c \mathcal{G}}=\{S \times] 0,1\left[\mid S \in \mathrm{S}_{\mathcal{G}}\right\} \cup\{\vartheta\},
$$

since $\mathcal{G}$ has no 0 -dimensional leaves. For technical reasons we allow $n$ to take the value 0 ; in this case $\mathbb{S}^{n-1}=\emptyset$ and $c \mathbb{S}^{n-1}=\{\vartheta\}$.

The same considerations apply to the $\infty$-cone $c_{\infty} \mathbb{S}^{n-1}=\mathbb{S}^{n-1} \times\left[0, \infty\left[/ \mathbb{S}^{n-1}\right.\right.$ $\times\{0\}$. In particular, $\mathbb{R}^{n}=c_{\infty} \mathbb{S}^{n-1}$. Notice that the map $f:\left(c \mathbb{S}^{n-1}, c \mathcal{G}\right) \rightarrow$ $\left(c_{\infty} \mathbb{S}^{n-1}, c_{\infty} \mathcal{G}\right)$ defined by $f[\theta, t]=[\theta, \tan (t \pi / 2)]$ is a foliated diffeomorphism.

The strata of a singular foliation are not necessarily manifolds. This leads to the following definition. In order to support an intersection cohomology, the stratification $\mathcal{S}_{\mathcal{F}}$ needs a certain amount of regularity and conicality (see [8] for the case of stratified pseudomanifolds).

1.2. Conical foliations. The definition is by induction on $m=\operatorname{dim} M$. A regular foliation is conical by definition.

If $m=1$, a conical foliation is regular, and either it is a foliation by points, or it has just one leaf. In the general case, a singular foliation $(M, \mathcal{F})$ is said to be a conical foliation if for any $x \in M$ there exists $n \in\{1,2, \ldots\}$ and a foliated diffeomorphism

$$
\varphi:\left(\mathbb{R}^{m-n} \times c \mathbb{S}^{n-1}, \mathcal{H} \times c \mathcal{G}\right) \rightarrow\left(U, \mathcal{F}_{U}\right)
$$

where

- $U \subset M$ is an open neighborhood of $x$,

- $\mathcal{H}$ is a regular foliation of $\mathbb{R}^{m-n}$,

- $\left(\mathbb{S}^{n-1}, \mathcal{G}\right)$ is a conical foliation without 0 -dimensional leaves, called the link of $x$, and

- $\varphi(0, \vartheta)=x$.

We shall say that $(U, \varphi)$ is a conical chart at $x$. Notice that if $S$ is the stratum containing $x$ then $\varphi^{-1}(S \cap U)=\mathbb{R}^{m-n} \times\{\vartheta\}$.

For a regular foliation the link is the empty set. 
The following examples well illustrate the situation in dimension 2 .

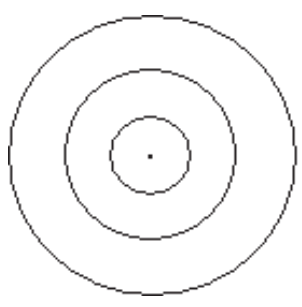

Conical

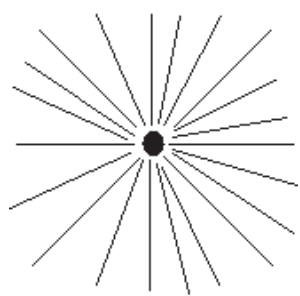

Not conical

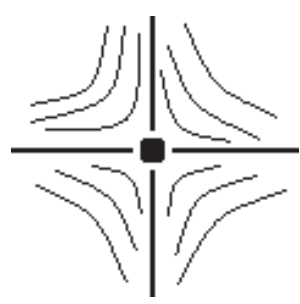

Not conical

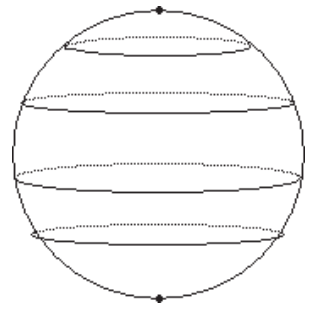

Conical

Although a point $x$ may have several charts the integer $n$ is an invariant: it is the codimension of the stratum containing $x$. This integer cannot be 1 since the conical foliation $\left(\mathbb{S}^{n-1}, \mathcal{G}\right)$ has no 0-dimensional leaves.

We will also consider manifolds with corners of the type $M \times\left[0,1\left[{ }^{p}, p>0\right.\right.$; they are foliated by foliations $\mathcal{F} \times \mathcal{I}$, where $\mathcal{I}$ is the foliation by points of $\left[0,1\left[{ }^{p}\right.\right.$. In fact, this is the restriction of the foliation $\mathcal{F} \times \mathcal{I}$ of $\left.M \times\right]-1,1\left[{ }^{p}\right.$, where $\mathcal{I}$ is the foliation by points of $]-1,1{ }^{p}$. We say the the foliation $\mathcal{F} \times \mathcal{I}$ of $M \times\left[0,1\left[{ }^{p}\right.\right.$ is conical if the foliation $\mathcal{F} \times \mathcal{I}$ of $\left.M \times\right]-1,1\left[^{p}\right.$ is. This is equivalent to the assumption that the foliation $\mathcal{F}$ of $M$ is conical.

1.2.1. Stratifications. In the compact case, the family of strata is finite, and locally finite in the general case. The closure $\bar{S}$ of a stratum $S \in \mathrm{S}_{\mathcal{F}}$ is a union of strata. We define an order $\preceq$ in the set of strata by $S_{1} \preceq S_{2}$ if $S_{1}, S_{2} \in \mathrm{S}_{\mathcal{F}}$ and $S_{1} \subset \bar{S}_{2}$.

The depth of $\mathbf{S}_{\mathcal{F}}$, written depth $\mathbf{S}_{\mathcal{F}}$, is defined to be the largest $i$ for which there exists a chain of strata $S_{0} \prec S_{1} \prec \cdots \prec S_{i}$. So, depth $\mathrm{S}_{\mathcal{F}}=0$ if and only if the foliation $\mathcal{F}$ is regular. Depth is always finite because of the local finiteness of $S_{\mathcal{F}}$. Moreover, depth $S_{\mathcal{F}_{U}} \leq \operatorname{depth} S_{\mathcal{F}}$ for any open subset $U \subset M$, and depth $\mathrm{S}_{\mathcal{G}}=\operatorname{depth} \mathrm{S}_{\mathcal{H} \times \mathcal{G}}<\operatorname{depth} \mathrm{S}_{\mathcal{H} \times c \mathcal{G}}$ (cf. 1.1.1).

The minimal strata are exactly the closed strata. An inductive argument on depth $S_{\mathcal{F}}$ shows that the maximal strata are those of dimension $m$ (just consider the local model (1)). They are called regular strata, and the others singular strata. Since the codimension of singular strata is at least 2, only one regular stratum $R_{\mathcal{F}}$ appears, which is an open dense subset. The union of the singular strata will be denoted by $\Sigma_{\mathcal{F}}$.

The restriction of $\mathcal{F}$ to a stratum $S$ defines a regular foliation $\mathcal{F}_{S}$. Moreover, if $S_{1} \prec S_{2}$ then $\operatorname{dim} \mathcal{F}_{S_{1}}<\operatorname{dim} \mathcal{F}_{S_{2}}$. So, the biggest leaves of $\mathcal{F}$ live in $R_{\mathcal{F}}$. The dimension $\operatorname{dim} \mathcal{F}$ of the foliation $\mathcal{F}$ is the dimension of these leaves, that is, $\operatorname{dim} \mathcal{F}=\operatorname{dim} \mathcal{F}_{R_{\mathcal{F}}}$. Notice that for any singular stratum $S$ we have the equality

$$
\operatorname{dim} \mathcal{F}-\operatorname{dim} \mathcal{F}_{S}=\operatorname{dim} \mathcal{G},
$$

where $\left(\mathbb{S}^{n-1}, \mathcal{G}\right)$ is the link of a point of $S$ (see (6)). 


\subsubsection{EXAMPLES.}

(a) Foliated bundles. Let $(N, \mathcal{N})$ be a foliation, $B$ a manifold, $\widetilde{B}$ its universal covering and $h: \pi_{1}(B) \rightarrow \operatorname{Diff}(N, \mathcal{N})$ a representation into the group of diffeomorphisms of $N$ preserving $\mathcal{N}$. A foliated bundle is the crossed product $M=\widetilde{B} \times{ }_{\pi_{1}(B)} N$ endowed with the foliation $\mathcal{F}$ whose leaves are obtained from $\widetilde{B} \times L, L \in \mathcal{N}$. This foliated bundle is conical. The dynamics of the leaves, strata, ... can be very complicated.

1. Put $B=\mathbb{S}^{1}, N=c_{\infty} \mathbb{S}^{n}, n>0, \mathcal{N}=c \mathcal{G}, \mathcal{G}$ the one-leaf foliation and $h(1)$ defined by $h(1)(u)=u / 2$. The minimal stratum $S=\mathbb{S}^{1}$ does not have a small saturated tubular neighborhood.

2. Put $B=\mathbb{S}^{1}, N=c \mathbb{S}^{2 n+1}, \mathcal{G}$ the Hopf foliation and $h(1)$ defined by

$$
h(1)\left[\left(z_{1}, \ldots, z_{n}\right), t\right]=\left[\left(e^{2 \pi i t} \cdot z_{1}, \ldots, e^{2 \pi i t} \cdot z_{n}\right), t\right]
$$

and $\mathcal{F}=c \mathcal{G}$. Here the leaves are diffeomorphic to cylinders (at irrational $t$-levels) or to tori (at non-zero rational $t$-levels) or to a circle (for $t=0$ ) and they are completely mixed up. The minimal stratum $S=\mathbb{S}^{1}$ has small saturated tubular neighborhoods, but they do not retract to $S$ through a foliated retraction.

(b) Singular riemannian foliations. A singular riemannian foliation, $\mathrm{SRF}$ for short, is a singular foliated manifold $(M, \mathcal{F})$ endowed with a riemannian metric such that every geodesic that is perpendicular to a leaf at one point remains perpendicular to every leaf it meets (cf. [12]). The restriction of $\mathcal{F}$ to each stratum $S$ of $S_{\mathcal{F}}$ is a regular riemannian foliation. The local structure of $\mathcal{F}$ can be described as follows (cf. [12] and [2]). Given $x \in M$ we can find a chart modeled on $\left(\mathbb{R}^{m-n} \times \mathbb{R}^{n}, \mathcal{H} \times \mathcal{K}\right)$ where $\left(\mathbb{R}^{m-n}, \mathcal{H}\right)$ is a simple foliation, $\left(\mathbb{R}^{n}, \mathcal{K}\right)$ is an $\mathrm{SRF}$ having the origin as unique 0-dimensional leaf and being invariant under homotheties.

The sphere $\mathbb{S}^{n-1}$ inherits a singular riemannian foliation $\mathcal{G}$ with $\mathcal{K}=c \mathcal{G}$ since $\mathcal{K}$ is invariant under homotheties [12]. We conclude that an SRF is a conical foliation.

(c) Isometric actions. The foliation determined by an isometric action induces a singular riemannian foliation and therefore a conical foliation. This example will be treated more extensively in the second section.

(d) The product. Given two conical foliated manifolds $\left(M_{1}, \mathcal{F}_{1}\right)$ and $\left(M_{2}, \mathcal{F}_{2}\right)$ their product $\left(M_{1} \times M_{2}, \mathcal{F}_{1} \times \mathcal{F}_{2}\right)$ is also conical.

Proof. We proceed by induction on depth $S_{\mathcal{F}_{1}}+\operatorname{depth} S_{\mathcal{F}_{2}}$. When this number is 0 the product foliation is conical since it is regular. The proof in the general case is just a local matter. Taking into account (b) it suffices to consider the product $\left(c \mathbb{S}^{n_{1}}, c \mathcal{G}_{1}\right) \times\left(c \mathbb{S}^{n_{2}}, c \mathcal{G}_{2}\right)$, where $\left(\mathbb{S}^{n_{1}}, \mathcal{G}_{1}\right)$ and $\left(\mathbb{S}^{n_{2}}, \mathcal{G}_{2}\right)$ are conical. By the induction hypothesis it suffices to check the local con- 
icality near $\left(\vartheta_{1}, \vartheta_{2}\right)$. We are going to construct a conical foliated manifold $\left(\mathbb{S}^{n_{1}+n_{2}+1}, \mathcal{G}\right)$ and a foliated embedding $\zeta:\left(c \mathbb{S}^{n_{1}+n_{2}+1}, c \mathcal{G}\right) \rightarrow\left(c \mathbb{S}^{n_{1}}, c \mathcal{G}_{1}\right) \times$ $\left(c \mathbb{S}^{n_{2}}, c \mathcal{G}_{2}\right)$ sending $\vartheta$ to $\left(\vartheta_{1}, \vartheta_{2}\right)$.

Since the foliation on the cone $c \mathbb{S}^{n_{i}}$ is tangent to each sphere $S^{n_{i}} \times\{t\}$, the submanifold $N=\left\{\left(\left[\theta_{1}, t_{1}\right],\left[\theta_{2}, t_{2}\right]\right) \in c \mathbb{S}^{n_{1}} \times c \mathbb{S}^{n_{2}} \mid t_{1}^{2}+t_{2}^{2}=1 / 2\right\}$ is a foliated submanifold. Moreover, the map

$$
\psi:(N \times] 0,1\left[,\left(c \mathcal{G}_{1} \times c \mathcal{G}_{2}\right)_{N} \times \mathcal{I}\right) \longrightarrow\left(c \mathbb{S}^{n_{1}} \times c \mathbb{S}^{n_{2}}, c \mathcal{G}_{1} \times c \mathcal{G}_{2}\right)
$$

defined by $\psi\left(\left[\theta_{1}, t_{1}\right],\left[\theta_{2}, t_{2}\right], t\right)=\left(\left[\theta_{1}, t \cdot t_{1}\right],\left[\theta_{2}, t \cdot t_{2}\right]\right)$ is a foliated embedding. We claim that $\left(N,\left(c \mathcal{G}_{1} \times c \mathcal{G}_{2}\right)_{N}\right)$ is conical. Again this is a local question, so it suffices to prove that $\left(N-\left\{t_{1}=0\right\},\left(\mathcal{G}_{1} \times \mathcal{G}_{2}\right)_{N-\left\{t_{1}=0\right\}}\right)$ is conical (and similarly for $\left.N-\left\{t_{2}=0\right\}\right)$. Since it is foliated diffeomorphic to $\left(\mathbb{S}^{n_{1}} \times\right] 0,1[\times$ $c \mathbb{S}_{2}^{n}, \mathcal{G}_{1} \times \mathcal{I} \times c \mathcal{G}_{2}$ ), where $\mathcal{I}$ is the 0 -dimensional foliation of ]0, 1 [, it suffices to apply the induction hypothesis to get the claim.

Notice that $N$ is diffeomorphic to the sphere $\mathbb{S}^{n_{1}+n_{2}+1}$ by the map $\left(\left[\theta_{1}, t_{1}\right],\left[\theta_{2}, t_{2}\right]\right) \mapsto\left(\sqrt{2} t_{1} \cdot \theta_{1}, \sqrt{2} t_{2} \cdot \theta_{2}\right)$. The induced foliation $\left(\mathbb{S}^{n_{1}+n_{2}+1}, \mathcal{G}\right)$ is conical. Under this diffeomorphism, the foliated embedding $\psi$ becomes the foliated embedding

$$
\zeta:\left(\mathbb{S}^{n_{1}+n_{2}+1} \times\right] 0,1[, \mathcal{G} \times \mathcal{I}) \rightarrow\left(c \mathbb{S}^{n_{1}} \times c \mathbb{S}^{n_{2}}, c \mathcal{G}_{1} \times c \mathcal{G}_{2}\right),
$$

defined by

$$
\zeta\left((u, v)=\left(x_{0}, \ldots, x_{n_{1}}, y_{0}, \ldots, y_{n_{2}}\right), t\right)=\left(\left[\frac{u}{\|u\|}, \frac{t \cdot\|u\|}{\sqrt{2}}\right],\left[\frac{v}{\|v\|}, \frac{t \cdot\|v\|}{\sqrt{2}}\right]\right) .
$$

But $\zeta$ extends to a foliated embedding $\zeta:\left(c \mathbb{S}^{n_{1}+n_{2}+1}, c \mathcal{G}\right) \rightarrow\left(c \mathbb{S}^{n_{1}} \times c \mathbb{S}^{n_{2}}\right.$, $\left.c \mathcal{G}_{1} \times c \mathcal{G}_{2}\right)$ by putting $\zeta(\vartheta)=\left(\vartheta_{1}, \vartheta_{2}\right)$. This ends the proof

The following result will be useful.

1.2.3. Lemma. Let $(M, \mathcal{F})$ and $\left(M^{\prime}, \mathcal{F}^{\prime}\right)$ be two conical foliated manifolds of the same dimension and let $f: M \times\left[0,1\left[\rightarrow M^{\prime} \times[0,1[\right.\right.$ be an embedding. If the restriction

$$
f:(M \times] 0,1[, \mathcal{F} \times \mathcal{I}) \rightarrow\left(M^{\prime} \times\right] 0,1\left[, \mathcal{F}^{\prime} \times \mathcal{I}\right)
$$

is foliated then so is

$$
f:\left(M \times\left[0,1[, \mathcal{F} \times \mathcal{I}) \rightarrow\left(M^{\prime} \times\left[0,1\left[, \mathcal{F}^{\prime} \times \mathcal{I}\right) .\right.\right.\right.\right.
$$

Proof. Notice first that when the two foliations are regular, the result comes directly from the local description of $f$.

Let $S \in \mathrm{S}_{\mathcal{F}}$ be a minimal stratum. From (3) there exists $S^{\prime} \in \mathrm{S}_{\mathcal{F}^{\prime}}$ with $\left.f(S \times] 0,1[) \subset S^{\prime} \times\right] 0,1\left[\right.$ and therefore $f\left(S \times\left[0,1[) \subset \overline{S^{\prime}} \times[0,1[\right.\right.$. We claim that

$$
f\left(S \times\left[0,1[) \subset S^{\prime} \times[0,1[.\right.\right.
$$

Indeed, suppose there exists $S_{0}^{\prime} \in \mathrm{S}_{\mathcal{F}^{\prime}}$ with $S_{0}^{\prime} \prec S^{\prime}$ and $f(S \times\{0\}) \cap$ $\left(S_{0}^{\prime} \times\{0\}\right) \neq \emptyset$. Since $f\left(M \times\left[0,1[)\right.\right.$ is an open subset of $M^{\prime} \times[0,1[$ we have 
$f(M \times] 0,1[) \cap\left(S_{0}^{\prime} \times\right] 0,1[) \neq \emptyset$. But this is not possible since

$$
f:(M \times] 0,1[, \mathcal{F} \times \mathcal{I}) \rightarrow\left(f(M \times] 0,1[), \mathcal{F}^{\prime} \times \mathcal{I}\right)
$$

is a foliated diffeomorphism and $S \times] 0,1\left[\right.$ is a minimal stratum of $\mathrm{S}_{\mathcal{F} \times \mathcal{I}}$.

We now proceed by induction on depth $S_{\mathcal{F}}$. If this depth is 0 then $\mathcal{F}$ is a regular foliation and the above considerations give $f\left(M \times\left[0,1[) \subset R^{\prime} \times[0,1[\right.\right.$, where $R^{\prime}$ is a regular stratum of $S_{\mathcal{F}^{\prime}}$. We get the result since the two foliations are regular.

Consider now the general case. Denote by $S_{\min }$ the union of the closed strata of $\mathcal{F}$. By induction hypothesis the restriction

$$
f:\left(\left(M-S_{\min }\right) \times\left[0,1[, \mathcal{F} \times \mathcal{I}) \rightarrow\left(M^{\prime} \times\left[0,1\left[, \mathcal{F}^{\prime} \times \mathcal{I}\right)\right.\right.\right.\right.
$$

is a foliated map.

Let now $S \in \mathrm{S}_{\mathcal{F}}$ be a singular stratum. We have seen that there exists $S^{\prime} \in \mathrm{S}_{\mathcal{F}^{\prime}}$ with $f\left(S \times\left[0,1[) \subset S^{\prime} \times[0,1\right.\right.$. It remains to prove that

$$
f:\left(S \times\left[0,1[, \mathcal{F} \times \mathcal{I}) \rightarrow\left(S^{\prime} \times\left[0,1\left[, \mathcal{F}^{\prime} \times \mathcal{I}\right) .\right.\right.\right.\right.
$$

This follows since the two foliations are regular.

1.3. Local blow-up. Consider a conical chart $(U, \varphi)$ at a point $x$ of a singular stratum $S$ of $M$. The composition map

$$
P_{\varphi}:\left(\mathbb{R}^{m-n} \times \mathbb{S}^{n-1} \times\left[0,1[, \mathcal{H} \times \mathcal{G} \times \mathcal{I}) \rightarrow\left(U, \mathcal{F}_{U}\right),\right.\right.
$$

defined by $P_{\varphi}(u, \theta, t)=\varphi(u,[\theta, t])$, is said to be a local blow-up. It is a foliated smooth map such that

- The restriction $\left.P_{\varphi}: \mathbb{R}^{m-n} \times \mathbb{S}^{n-1} \times\right] 0,1[\rightarrow U-S$ is a diffeomorphism.

- The restriction $P_{\varphi}: \mathbb{R}^{m-n} \times \mathbb{S}^{n-1} \times\{0\} \rightarrow U \cap S$ is a fiber bundle whose fiber is $\mathbb{S}^{n-1}$.

In other words, the local blow-up replaces each point of the minimal stratum by a link.

The following result shows that the local blow-up is essentially unique and that the link of $x$ is also unique. The proposition was announced in [15] without proof.

1.3.1. Proposition. Let $\left(U_{1}, \varphi_{1}\right),\left(U_{2}, \varphi_{2}\right)$ be two charts at a point $x$ of $M$ with $U_{1} \subset U_{2}$. There exists a unique embedding

$$
\Phi_{1,2}: \mathbb{R}^{m-n} \times \mathbb{S}^{n-1} \times\left[0,1\left[\rightarrow \mathbb{R}^{m-n} \times \mathbb{S}^{n-1} \times[0,1[\right.\right.
$$

making the following diagram commutative: 


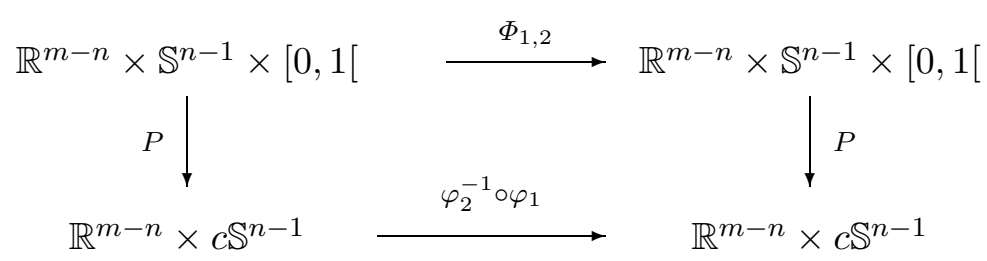

where $P(u, \theta, t)=(u,[\theta, t])$. Moreover, if the two charts are conical, modeled respectively on $\left(\mathbb{R}^{m-n} \times c \mathbb{S}^{n-1}, \mathcal{H}_{1} \times c \mathcal{G}_{1}\right)$ and $\left(\mathbb{R}^{m-n} \times c \mathbb{S}^{n-1}, \mathcal{H}_{2} \times c \mathcal{G}_{2}\right)$, then

$\Phi_{1,2}:\left(\mathbb{R}^{m-n} \times \mathbb{S}^{n-1} \times\left[0,1\left[, \mathcal{H}_{1} \times \mathcal{G}_{1} \times \mathcal{I}\right) \rightarrow\left(\mathbb{R}^{m-n} \times \mathbb{S}^{n-1} \times\left[0,1\left[, \mathcal{H}_{2} \times \mathcal{G}_{2} \times \mathcal{I}\right)\right.\right.\right.\right.$ is a foliated embedding.

Proof. Notice that $\varphi_{2}^{-1} \circ \varphi_{1}: \mathbb{R}^{m-n} \times c \mathbb{S}^{n-1} \rightarrow \mathbb{R}^{m-n} \times c \mathbb{S}^{n-1}$ is an embedding.

Uniqueness. It follows from density of $\left.\mathbb{R}^{m-n} \times \mathbb{S}^{n-1} \times\right] 0,1[$ and from the fact that $\left.P: \mathbb{R}^{m-n} \times \mathbb{S}^{n-1} \times\right] 0,1\left[\rightarrow \mathbb{R}^{m-n} \times\left(c \mathbb{S}^{n-1}-\{\vartheta\}\right)\right.$ is a diffeomorphism.

Existence. Denote by $(f, g): \mathbb{R}^{m-n} \times \mathbb{R}^{n} \rightarrow \mathbb{R}^{m-n} \times \mathbb{R}^{n}$ the embedding $\varphi_{2}^{-1} \circ \varphi_{1}$. The components $f$ and $g$ are smooth with $g(0)=0$. So, the map $h: \mathbb{R}^{m-n} \times \mathbb{S}^{n-1} \times\left[0,1\left[\rightarrow \mathbb{R}^{n}\right.\right.$ defined by $h(u, \theta, t)=g(u, t \cdot \theta) / t$ is smooth and without zeroes. Finally, we define

$$
\Phi_{1,2}(u, \theta, t)=\left(f(u, t \cdot \theta), \frac{h(u, \theta, t)}{\|h(u, \theta, t)\|}, t \cdot\|h(u, \theta, t)\|\right) .
$$

Embedding. Notice first the following. Since $(f, g)$ is an embedding with $g(0)=0$, each $g(u,-): \mathbb{R}^{n} \rightarrow \mathbb{R}^{n}$ is an embedding. Let $G_{u}$ be its derivative at 0 , which is an isomorphism. By construction we have $h(u, \theta, 0)=$ $G_{u}(\theta) /\left\|G_{u}(\theta)\right\|$. So, each restriction $\Phi_{1,2}:\{u\} \times \mathbb{S}^{n-1} \times\{0\} \rightarrow\{f(u, 0)\} \times$ $\mathbb{S}^{n-1} \times\{0\}$ is a diffeomorphism.

Now, consider two points (resp. two vectors) on $\mathbb{R}^{m-n} \times \mathbb{S}^{n-1} \times[0,1[$ sent by $\Phi_{1,2}$ to the same point (resp. vector). Since $\varphi_{2}^{-1} \circ \varphi_{1}$ is an embedding, they live on a fiber $\{u\} \times \mathbb{S}^{n-1} \times\{0\}$. Since $G_{u}$ is an isomorphism, we get the claim.

Foliated. The embedding $\Phi_{1,2}$ extends the foliated map $\varphi_{2}^{-1} \circ \varphi_{1}$. We apply Lemma 1.2.3.

An important consequence of this proposition is that the links $\left(\mathbb{S}^{n-1}, \mathcal{G}\right)$ of two points of the same stratum $S$ are foliated diffeomorphic. We shall write $\mathcal{G}_{S}=\mathcal{G}$.

\section{FOLIATIONS DETERMINED BY AN ABELIAN ISOMETRIC ACTION}

In this paper we deal with an abelian isometric action $\Phi: G \times M \rightarrow M$ on a compact smooth manifold $M$. As we study the induced foliation $\mathcal{F}$, we 
may assume that the group $G$ is connected; it suffices to replace $G$ by the connected component $G_{0}$ of the identity.

For technical reasons, we also need to work with non-compact manifolds. Tame actions are introduced for this purpose.

2.1. Tame actions. A smooth action $\Phi: G \times M \rightarrow M$ of an abelian Lie group $G$ on a smooth manifold $M$ is tame if it extends to a smooth action $\Phi: K \times M \rightarrow M$ where $K$ is an abelian compact Lie group containing $G$. We say that $K$ is a tamer group of $G$. When $M$ is compact, this notion is equivalent to that of isometric action (cf. [9]).

When necessary, we can suppose that the group $G$ is dense in $K$.

The restriction of the action of $G$ to a $K$-invariant submanifold of $M$ is again a tame action. For a subgroup $H \subset G$ the restriction $\Phi: H \times M \rightarrow M$ is also a tame action.

The connected components of the orbits of the tame action $\Phi$ determine a foliation $\mathcal{F}$ on $M$. Since the action $\Phi: G \times M \rightarrow M$ is isometric, $\mathcal{F}$ is a conical foliation (cf. 1.2.2(b)).

2.1.1. Three particular actions. We now describe the particular actions we shall use.

(a) The action $\Xi: G \times K \rightarrow K$ defined by $\Xi(g, k)=g \cdot k$. This action is tame since it extends to $\Xi: K \times K \rightarrow K$ defined by $\Xi(g, k)=g \cdot k$. We write $\mathcal{F}_{K}$ for the induced foliation; then $\operatorname{dim} \mathcal{F}_{K}=\operatorname{dim} G$. For each $u \in \mathfrak{g}$, the Lie algebra of $G$, we write $X_{u} \in \mathcal{X}(K)$ for the associated fundamental vector field.

(b) The action $\Psi: G \times K / H \rightarrow K / H$ defined by $\Psi(g, k H)=(g \cdot k) H$, where $H \subset K$ a closed subgroup. This action is tame since it extends to $\Psi: K \times K / H \rightarrow K / H$ defined by the same formula. We write $\mathcal{F}_{K / H}$ for the induced foliation, with $\operatorname{dim} \mathcal{F}_{K / H}=\operatorname{dim} G H / H=\operatorname{dim} G /(G \cap H)$. For each $u \in \mathfrak{g}$ we write $Y_{u} \in \mathcal{X}(K / H)$ for the associated fundamental vector field. If $\pi: K \rightarrow K / H$ is the canonical projection then $\pi_{*} X_{u}=Y_{u}$.

(c) The action $\Gamma: G \cap H \times H \rightarrow H$ defined by $\Gamma(g, h)=g \cdot h$. This action is tame since it extends to $\Gamma: H \times H \rightarrow H$ defined by $\Gamma(g, h)=g \cdot h$. We write $\mathcal{F}_{H}$ for the induced foliation, with $\operatorname{dim} \mathcal{F}_{H}=\operatorname{dim} G \cap H$. For each $u \in \mathfrak{g} \cap \mathfrak{h}$ we write $Z_{u} \in \mathcal{X}(H)$ for the associated fundamental vector field. Here $\mathfrak{h}$ is the Lie algebra of $H$.

2.2. Twisted product. This is the first geometrical tool we use for the study of $\mathcal{F}$.

Let $K$ be a connected compact abelian Lie group and $G$ a connected subgroup. Suppose that $G$ is dense in $K$. Consider an orthogonal action $\Theta: H \times \mathbb{R}^{n} \rightarrow \mathbb{R}^{n}$ where $H$ is a compact subgroup of $K$. The twisted 
product $K \times_{H} \mathbb{R}^{n}$ is the quotient of $K \times \mathbb{R}^{n}$ by the equivalence relation $\left(k \cdot h^{-1}, \Theta(h, z)\right) \sim(k, z)$. The element of the twisted product corresponding to $(k, z)$ is denoted by $\langle k, z\rangle$. It is a manifold endowed with the action

$$
\Phi: G \times\left(K \times_{H} \mathbb{R}^{n}\right) \rightarrow K \times_{H} \mathbb{R}^{n}
$$

defined by $\Phi(g,\langle k, z\rangle)=\langle g \cdot k, z\rangle$. It is clearly a tame action and we denote by $\mathcal{F}_{\text {tw }}$ the induced conical foliation.

The restriction $\Theta: G \cap H \times \mathbb{R}^{n} \rightarrow \mathbb{R}^{n}$ is also a tame action. The induced conical foliation is denoted by $\mathcal{F}_{\mathbb{R}^{n}} ; H$ is a tamer group. The couple $\left(\mathbb{R}^{n}, \mathcal{F}_{\mathbb{R}^{n}}\right)$ is a slice of the twisted product.

The product $K \times \mathbb{R}^{n}$ is endowed with the conical foliation $\mathcal{F}_{K} \times \mathcal{F}_{\mathbb{R}^{n}}$. The natural projection $R: K \times \mathbb{R}^{n} \rightarrow K \times_{H} \mathbb{R}^{n}$ gives the relations $\mathcal{F}_{\text {tw }}=$ $R_{*}\left(\mathcal{F}_{K} \times \mathcal{F}_{\mathbb{R}^{n}}\right)$ and

$$
\mathrm{S}_{\mathcal{F}_{\mathrm{tw}}}=\left\{R(K \times S) \mid S \in \mathrm{S}_{\mathcal{F}_{\mathbb{R}^{n}}}\right\}=R\left(\{K\} \times \mathrm{S}_{\mathcal{F}_{\mathbb{R}^{n}}}\right) .
$$

2.3. Tubular neighborhood. This is the second geometrical tool we use for the study of $\mathcal{F}$.

Consider a singular stratum $S$ of $\mathrm{S}_{\mathcal{F}}$. Since $\operatorname{dim} G(k \cdot x)=\operatorname{dim} G(x)$ for all $k \in K$ and $x \in S, S$ is a $K$-invariant proper submanifold of $M$. It has a $K$-invariant tubular neighborhood $\left(T, \tau, S, \mathbb{R}^{n}\right)$ whose structural group is $O(n)$. This means that $\tau$ is $K$-invariant and there exists an atlas $\mathcal{B}$ such that for any two charts $(U, \varphi),(V, \psi) \in \mathcal{B}$ and $k \in K$ the composition

$$
\psi \circ k \circ \varphi^{-1}:\left(U \cap\left(k^{-1} V\right)\right) \times \mathbb{R}^{n} \rightarrow((k U) \cap V) \times \mathbb{R}^{n}
$$

is of the form $(x, u) \mapsto\left(k \cdot x, A_{x, k}(u)\right)$ with $A_{x, k} \in O(n)$.

Recall that the following smooth maps are associated with this neighborhood:

- The radius map $\varrho: T \rightarrow[0,1[$ defined fiberwise by $[x, t] \mapsto t$. Each $t \neq 0$ is a regular value of $\varrho$. The pre-image $\varrho^{-1}(0)$ is $S$. This map is $K$-invariant, that is, $\varrho(k \cdot z)=\varrho(z)$.

- The contraction $H: T \times[0,1] \rightarrow T$ defined fiberwise by $([x, t], r) \mapsto$ $[x, r t]$. The restriction $H_{t}: T \rightarrow T$ is an embedding for each $t \neq 0$ and $H_{0} \equiv \tau$. We shall write $H(z, t)=t \cdot z$. This map is $K$-invariant, that is, $t \cdot(k \cdot z)=k \cdot(t \cdot z)$.

These two maps are related by $\varrho(t \cdot z)=t \varrho(z)$.

The hypersurface $D=\varrho^{-1}(1 / 2)$ is the tube of the tubular neighborhood. It is a $K$-invariant submanifold of $T$. Notice that the smooth map

$$
\nabla: D \times[0,1[\rightarrow T
$$

defined by $\nabla(z, t)=(2 t) \cdot z$ is $K$-equivariant, where $K$ acts trivially on the $[0,1$ [-factor. Its restriction $\nabla: D \times] 0,1[\rightarrow T-S$ is a $K$-equivariant diffeomorphism, and $\nabla: D \times\{0\} \equiv D \rightarrow S$ is $\tau$. 
The foliation $\mathcal{F}$ induces a tame foliation on the fibers of $\tau$. To see that, let $G_{S}=G_{x_{0}}$ be the isotropy subgroup of some (and hence every) point $x_{0} \in S$. This group acts effectively on $\tau^{-1}\left(x_{0}\right)$. The trace on $\tau^{-1}\left(x_{0}\right)$ of $\mathcal{F}$ is given by the action of $G_{S}$. Using a chart of the atlas $\mathcal{B}$ we identify $\tau^{-1}\left(x_{0}\right)$ with $\mathbb{R}^{n}$. The induced foliated manifold $\left(\mathbb{R}^{n}, \mathcal{F}_{\mathbb{R}^{n}}\right)$ is the slice of the tube.

The action of $G_{S}$ induces an orthogonal action $\Theta: G_{S} \times \mathbb{S}^{n-1} \rightarrow \mathbb{S}^{n-1}$. This action is effective and tame. Let $\mathcal{G}_{S}$ be the induced conical foliation. In fact, $\left(\mathbb{S}^{n-1}, \mathcal{G}_{S}\right)$ is the link of $S$. The formula (2) becomes

$$
\operatorname{dim} \mathcal{F}=\operatorname{dim} \mathcal{F}_{S}+\operatorname{dim} \mathcal{G}_{S} .
$$

The tubular neighborhood gives rise to a local blow-up:

2.3.1. Proposition. Given a conical chart $(U, \varphi) \in \mathcal{B}$ there exists a commutative diagram

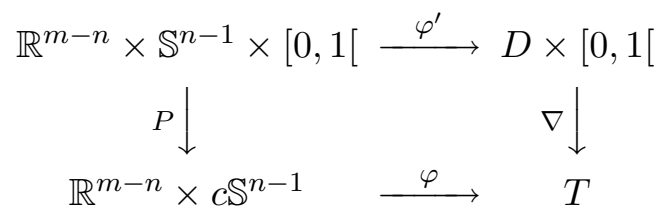

where $\varphi^{\prime}:\left(\mathbb{R}^{m-n} \times \mathbb{S}^{n-1} \times[0,1[, \mathcal{H} \times \mathcal{G} \times \mathcal{I}) \rightarrow(D \times[0,1[, \mathcal{F} \times \mathcal{I})\right.$ is a foliated embedding.

Proof. The existence of $\varphi^{\prime}$ and the commutativity $\tau \circ \varphi^{\prime}=\varphi \circ P$ are guaranteed by Lemma 1.3.1. We also know that the restriction

$\varphi^{\prime}=\nabla_{\min }^{-1} \circ \varphi \circ P:\left(\mathbb{R}^{m-n} \times \mathbb{S}^{n-1} \times\right] 0,1[, \mathcal{H} \times \mathcal{G} \times \mathcal{I}) \rightarrow(D \times] 0,1[, \mathcal{F} \times \mathcal{I})$

is a foliated embedding. Applying Lemma 1.2 .3 we conclude that $\varphi^{\prime}:\left(\mathbb{R}^{m-n}\right.$ $\times \mathbb{S}^{n-1} \times[0,1[, \mathcal{H} \times \mathcal{G} \times \mathcal{I}) \rightarrow(D \times[0,1[, \mathcal{F} \times \mathcal{I})$ is a foliated embedding.

2.4. Molino's blow-up. Molino's blow-up [12] of the foliation $\mathcal{F}$ produces a new foliation $\widehat{\mathcal{F}}$ of the same kind but of smaller depth. The main idea is to replace each point of closed strata by the fiber of a convenient tubular neighborhood. But in order to avoid the boundary that appears in this procedure, we take the double.

We suppose depth $S_{\mathcal{F}}>0$. Let $S_{\text {min }}$ be the union of the closed (minimal) strata and choose a disjoint family $T_{\min }$ of $K$-invariant tubular neighborhoods of the closed strata. The union of the associated tubes is denoted by $D_{\min }$. Notice that the induced map $\left.\nabla_{\min }: D_{\min } \times\right] 0,1\left[\rightarrow T_{\min }-S_{\min }\right.$ is a $K$-equivariant diffeomorphism. The blow-up of $M$ is the manifold

$$
\widehat{M}=\left\{\left(D_{\min } \times\right]-1,1[) \amalg\left(\left(M-S_{\min }\right) \times\{-1,1\}\right)\right\} / \sim,
$$


where $(z, t) \sim\left(\nabla_{\min }(z,|t|), t /|t|\right)$, and the map $\mathcal{L}: \widehat{M} \rightarrow M$ is defined by

$$
\mathcal{L}(v)= \begin{cases}\nabla_{\min }(z,|t|) & \text { if } \left.v=(z, t) \in D_{\min } \times\right]-1,1[, \\ z & \text { if } v=(z, j) \in\left(M-S_{\min }\right) \times\{-1,1\} .\end{cases}
$$

Notice that $\mathcal{L}$ is a continuous map whose restriction $\mathcal{L}: \widehat{M}-\mathcal{L}^{-1}\left(S_{\min }\right) \rightarrow$ $M-S_{\min }$ is a $K$-equivariant smooth trivial 2-covering.

Since $\nabla_{\min }$ is $K$-equivariant, $\Phi$ induces an action $\widehat{\Phi}: K \times \widehat{M} \rightarrow \widehat{M}$ by requiring that the blow-up $\mathcal{L}$ is $K$-equivariant. The open submanifolds $\mathcal{L}^{-1}\left(T_{\min }\right)$ and $\mathcal{L}^{-1}\left(T_{\min }-S_{\text {min }}\right)$ are clearly $K$-diffeomorphic to $\left.D_{\min } \times\right]-1,1[$ and $D_{\min } \times(]-1,0[\cup] 0,1[)$ respectively. Notice that the depth of the restrictions of $\mathcal{F}$ to $D_{\min }, T_{\min }-S_{\min }$ and $M_{\min }-S_{\text {min }}$ is strictly smaller than $\operatorname{depth} S_{\mathcal{F}}$.

The restriction $\widehat{\Phi}: G \times \widehat{M} \rightarrow \widehat{M}$ is a tame action, whose tamer is just $\widehat{\Phi}: K \times \widehat{M} \rightarrow \widehat{M}$. The induced foliation is $\widehat{\mathcal{F}}$. The associated foliations $\mathcal{F}$ and $\widehat{\mathcal{F}}$ are related by $\mathcal{L}$ which is a foliated map. Moreover, if $S$ is a non-minimal stratum of $\mathrm{S}_{\mathcal{F}}$ then there exists a unique stratum $S^{\prime}$ of $\mathrm{S}_{\widehat{\mathcal{F}}}$ such that $\mathcal{L}^{-1}(S) \subset S^{\prime}$. The family $\left\{S^{\prime} \mid S \in \mathrm{S}_{\mathcal{F}}\right\}$ covers $\widehat{M}$ and satisfies $S_{1} \prec S_{2} \Leftrightarrow S_{1}^{\prime} \prec S_{2}^{\prime}$. We deduce the important property

$$
\operatorname{depth} S_{\widehat{\mathcal{F}}}<\operatorname{depth} S_{\mathcal{F}}
$$

2.5. Orbit type stratification. Given an action $\Phi: K \times M \rightarrow M$, the points of $M$ are classified by the equivalence relation:

$$
x \sim y \Leftrightarrow K_{x}=K_{y} .
$$

The induced partition $\mathrm{S}_{\Phi}$ is the orbit type stratification of $M$ (see for example [3]). The elements of this stratification are connected $K$-invariant submanifolds, called ot-strata. This stratification is finer than the stratification $\mathrm{S}_{\overline{\mathcal{F}}}$ defined by the action $\Phi: K \times M \rightarrow M$, but it has similar properties, in particular, $\left(\mathrm{S}_{\Phi}, \prec\right)$ is a poset with finite depth.

Since an ot-stratum is a $K$-invariant submanifold, it has an invariant neighborhood. A blow-up can be constructed as in the previous framework: the ot-blow-up. We write $\mathcal{N}: \widetilde{M} \rightarrow M$ for the ot-blow-up, which is a $K$ equivariant continuous map relative to an action $\widetilde{\Phi}: K \times \widetilde{M} \rightarrow \widetilde{M}$. We have

$$
\operatorname{depth} S_{\widetilde{\Phi}}<\operatorname{depth} S_{\Phi}
$$

\section{BASIC INTERSECTION COHOMOLOGY}

The basic cohomology of a foliated space is the right cohomological tool to study the transverse structure of foliations. A conical foliation has a transverse structure which reminds the stratified pseudomanifolds of [8], and for this kind of singular spaces the best adapted cohomological tool is 
the intersection cohomology. In this section we mix up the two ingredients and we introduce the basic intersection cohomology.

For the rest of this section, we fix a conical foliated manifold $(M, \mathcal{F})$.

A differential form defined on the regular stratum may have a wild behavior relative to the singular strata. But there are some forms with a good relation to the singular part. These are the perverse forms, and from them we are going to construct the basic intersection cohomology.

There are several ways to define perverse forms: using a system of tubular neighborhoods (cf. [5]), a global blow-up (cf. [14]) etc.; in this work we introduce them in a more intrinsic way, using the local blow-ups we have already considered. This definition was formulated for the first time in [15]; we recall it below and prove some properties, some of which were just stated there without proof.

We are going to deal with differential forms on products (manifold) $\times$ $\left[0,1\left[{ }^{p} ;\right.\right.$ these forms are restrictions of differential forms defined on (manifold) $\times]-1,1\left[^{p}\right.$.

3.1. Perverse forms. Perverse forms are differential forms defined on the regular stratum $R_{\mathcal{F}}$ which are extendable through local blow-ups.

The differential complex $\Pi_{\mathcal{F}}^{*}\left(M \times\left[0,1\left[^{p}\right)\right.\right.$ of perverse forms on $M \times\left[0,1\left[^{p}\right.\right.$ is introduced by induction on depth $S_{\mathcal{F}}$. When this depth is 0 then

$$
\Pi_{\mathcal{F}}^{*}\left(M \times\left[0,1\left[^{p}\right)=\Omega^{*}\left(M \times\left[0,1\left[^{p}\right) .\right.\right.\right.\right.
$$

Consider now the general case. Let $M$ be a foliated manifold with a conical foliation of depth $k$. A perverse form on $M \times\left[0,1\left[^{p}\right.\right.$ is a differential form $\omega$ defined on $R_{\mathcal{F}} \times\left[0,1{ }^{p} \subset M \times\left[0,1{ }^{p}\right.\right.$ such that, for any conical chart $(U, \varphi)$, there exists a perverse form $\omega_{\varphi}$ on $\left(\mathbb{R}^{m-n} \times \mathbb{S}^{n-1} \times\left[0,1\left[{ }^{p+1}, \mathcal{H} \times \mathcal{G} \times \mathcal{I}\right)\right.\right.$ with

$$
\left.\omega_{\varphi}=\left(P_{\varphi} \times \text { identity }_{\left[0,1\left[{ }^{p}\right.\right.}\right)^{*} \omega \quad \text { on } \mathbb{R}^{m-n} \times R_{\mathcal{G}} \times\right] 0,1\left[\times\left[0,1\left[{ }^{p} .\right.\right.\right.
$$

Notice that this condition makes sense since

(i) the restriction of the local blow-up

$$
P_{\varphi}: \underbrace{\left.\mathbb{R}^{m-n} \times R_{\mathcal{G}} \times\right] 0,1[}_{\text {reg. stratum of } \mathbb{R}^{m-n} \times c \mathbb{S}^{n-1}} \rightarrow \underbrace{U \cap R_{\mathcal{F}}}_{\text {reg. stratum of } U}
$$

is a diffeomorphism,

(ii) the foliation $\mathcal{H} \times \mathcal{G}$ of $\mathbb{R}^{m-n} \times \mathbb{S}^{n-1}$ is of depth $k-1$.

We notice that

$$
P_{\varphi}^{*}: \Pi_{\mathcal{F}}^{*}(U) \rightarrow \Pi_{\mathcal{H} \times \mathcal{G} \times \mathcal{I}}^{*}\left(\mathbb{R}^{m-n} \times \mathbb{S}^{n-1} \times[0,1[)\right.
$$

is a differential graded commutative algebra (dgca for short) isomorphism.

The complex $\Pi_{\mathcal{F}}^{*}(M)$ is a dgca since $(\omega+\eta)_{\varphi}=\omega_{\varphi}+\eta_{\varphi},(\omega \wedge \eta)_{\varphi}=\omega_{\varphi} \wedge \eta_{\varphi}$ and $(d \omega)_{\varphi}=d \omega_{\varphi}$. 


\subsubsection{REMARKS.}

(a) The notion of perverse form depends on the foliation $\mathcal{F}$ through the stratification $\mathrm{S}_{\mathcal{F}}$.

(b) A local blow-up produces a factor [0,1[. Further desingularization would produce extra $\left[0,1\left[\right.\right.$ factors. This is the reason for introducing $\left[0,1\left[^{p}\right.\right.$ directly.

(c) The perversion condition does not depend on the choice of the conical chart. In fact, if (9) is satisfied for some conical chart then it is also satisfied for any other conical chart (cf. Proposition 1.3.1).

(d) The local blow-up of the cone $c \mathbb{S}^{n-1}$ is essentially the product $\mathbb{S}^{n-1} \times$ $\left[0,1\left[\right.\right.$. So, we have the natural identification $\Pi_{\mathcal{G}}^{*}\left(\mathbb{S}^{n-1} \times\left[0,1[)=\Pi_{c \mathcal{G}}^{*}\left(c \mathbb{S}^{n-1}\right)\right.\right.$ given by $\omega \mapsto \omega_{\left.\mid R_{\mathcal{G}} \times\right] 0,1[}$.

(e) Given a tubular neighborhood $\tau: T \rightarrow S$ of a singular stratum (cf. 2.3), the map $\nabla: D \times\left[0,1\left[\rightarrow T\right.\right.$ gives an isomorphism between $\Pi_{\mathcal{F}}^{*}(T)$ and $\Pi_{\mathcal{F} \times \mathcal{I}}^{*}(D \times[0,1[)$ (cf. Propositions 2.3.1 and 1.3.1).

(f) Let us illustrate this notion with an example. Consider the isometric action $\Phi: \mathbb{R} \times \mathbb{S}^{7} \rightarrow \mathbb{S}^{7}$ defined by

$$
\Phi\left(t,\left(z_{0}, z_{1}, z_{2}, z_{3}\right)\right)=\left(e^{a \pi i t} z_{0}, e^{b \pi i t} z_{1}, e^{c \pi i t} z_{2}, z_{3}\right)
$$

with $(a, b, c) \neq(0,0,0)$. Recall that the induced foliation $\mathcal{F}$ is conical (cf. 1.2.2(b)). We can view $\mathbb{S}^{7}$ as the join $\mathbb{S}^{5} * \mathbb{S}^{1}$ where $\mathbb{R}$ acts freely on the first factor, inducing the foliation $\mathcal{G}$, and trivially on the second factor. There is just one singular stratum, namely $\mathbb{S}^{1}$, whose link is $\left(\mathbb{S}^{5}, \mathcal{G}\right)$. This stratum has a "global conical chart" $\left(\mathbb{S}^{1} \times c \mathbb{S}^{5}, \mathcal{I} \times c \mathcal{G}\right)$, so

$$
\Pi_{\mathcal{G}}^{*}\left(\mathbb{S}^{7}\right)=\Omega^{*}\left(\mathbb{S}^{5} \times \mathbb{D}^{2}\right) .
$$

(g) There are differential forms on $R_{\mathcal{F}}$ which are not perverse. Any differential form $\omega$ on $M$ is perverse, that is, $\Omega^{*}(M) \subset \Pi_{\mathcal{F}}^{*}(M)$. In fact, we have $\omega_{\varphi}=P_{\varphi}^{*} \omega$ on $\mathbb{R}^{n-m} \times \mathbb{S}^{n-1} \times[0,1[$ for any conical chart $(U, \varphi)$.

Proof. For the first part consider a smooth function on $R_{\mathcal{F}}$ going to infinity when approaching the singular part. For the second part we proceed by induction on depth $S_{\mathcal{F}}$. Let $\omega \in \Pi_{\mathcal{F}}^{*}\left(M \times\left[0,1\left[{ }^{p}\right)\right.\right.$. For a conical chart $(U, \varphi)$, $\omega_{\varphi}=P_{\varphi}^{*} \omega \in \Omega^{*}\left(\mathbb{R}^{m-n} \times \mathbb{S}^{n-1} \times\left[0,1\left[{ }^{p+1}\right)\right.\right.$. Then we apply the induction hypothesis.

(h) An open foliated embedding $f:\left(M_{1}, \mathcal{F}_{1}\right) \rightarrow\left(M_{2}, \mathcal{F}_{2}\right)$ of foliated conical manifolds induces a dgca operator $f^{*}: \Pi_{\mathcal{F}_{2}}^{*}\left(M_{2}\right) \rightarrow \Pi_{\mathcal{F}_{1}}^{*}\left(M_{1}\right)$.

Proof. The embedding $f$ preserves conical charts and therefore perverse forms.

3.2. Perverse degree. The amount of transversality of a perverse form $\omega \in \Pi_{\mathcal{F}}^{*}(M)$ with respect to a singular stratum $S$ is measured by the perverse 
degree $\|\omega\|_{S}$. We first define the local perverse degree $\|\omega\|_{U}$ for a conical chart $(U, \varphi)$ at a point of $S$.

Notice that a local blow-up replaces $U \cap S$ by $\mathbb{R}^{m-n} \times R_{\mathcal{G}} \times\{0\}$ and that the restriction

$$
P_{\varphi}: \mathbb{R}^{m-n} \times R_{\mathcal{G}} \times\{0\} \rightarrow U \cap S
$$

is (isomorphic to) a trivial bundle. Since $\omega$ is a perverse form, the differential form $\varphi^{*} \omega$ (living on $\left.\mathbb{R}^{m-n} \times R_{\mathcal{G}} \times\right] 0,1\left[\right.$ ) extends to a differential form $\omega_{\varphi}$ (living on $\mathbb{R}^{m-n} \times R_{\mathcal{G}} \times\left[0,1[)\right.$. The perverse degree $\|\omega\|_{U}$ is the vertical degree of $\omega_{\varphi}$ relative to the added part, that is, the fibration (11).

Namely, when $\omega_{\varphi}=0$ on $\mathbb{R}^{m-n} \times R_{\mathcal{G}} \times\{0\}$ we put $\|\omega\|_{U}=-\infty$, and in the other cases

$$
\|\omega\|_{U}=\min \left\{\begin{array}{l|l}
k \in \mathbb{N} & \begin{array}{l}
\omega_{\varphi}\left(u_{0}, \ldots, u_{k}, \ldots\right) \equiv 0 \\
\text { for each family }\left\{u_{0}, \ldots, u_{k}\right\} \\
\text { of vectors tangent to the fibers of } \\
P_{\varphi}: \mathbb{R}^{m-n} \times R_{\mathcal{G}} \times\{0\} \rightarrow U \cap S
\end{array}
\end{array}\right\} .
$$

This number does not depend on the choice of the conical chart.

Proof. Let $\left(U, \varphi_{1}\right)$ and $\left(U, \varphi_{2}\right)$ be two conical charts. From Proposition 1.3.1 we know that $\omega_{\varphi_{1}}=\Phi_{1,2}^{*} \omega_{\varphi_{2}}$ and the restriction $\Phi_{1,2}: \mathbb{R}^{m-n} \times$ $R_{\mathcal{G}} \times\{0\} \rightarrow \mathbb{R}^{m-n} \times R_{\mathcal{G}} \times\{0\}$ is a diffeomorphism. This implies that $\|\omega\|_{U}^{\varphi_{1}}=\|\omega\|_{U}^{\varphi_{2}}$.

Finally, we define the perverse degree $\|\omega\|_{S}$ by

$$
\|\omega\|_{S}=\sup \left\{\|\omega\|_{U} \mid(U, \varphi) \text { is a conical chart at a point of } S\right\} .
$$

For two perverse forms $\omega$ and $\eta$ and a singular stratum $S$ we have

$$
\|\omega+\eta\|_{S} \leq \max \left\{\|\omega\|_{S},\|\eta\|_{S}\right\}, \quad\|\omega \wedge \eta\|_{S} \leq\|\omega\|_{S}+\|\eta\|_{S} .
$$

For a perverse form $\omega$ the perverse degree is smaller than the usual degree and satisfies

$$
\|\omega\|_{S} \leq \operatorname{dim} R_{\mathcal{G}_{S}}=\operatorname{codim}_{M} S-1 .
$$

\subsubsection{REMARKS.}

(a) The notion of perverse degree depends on the foliation $\mathcal{F}$ through the stratification $S_{\mathcal{F}}$.

(b) The perverse degree of a differential form on $M$ is $-\infty$ or 0 (cf. 3.1.1(g)).

(c) Consider $\mathbb{S}^{n-1}$ endowed with a conical foliation without 0-dimensional leaves, and the disk $c \mathbb{S}^{n-1}$ endowed with the induced conical foliation. Then the perverse degree of a form $\omega \in \Pi_{c \mathcal{G}}^{*}\left(c \mathbb{S}^{n-1}\right)=\Pi_{\mathcal{G} \times \mathcal{I}}^{*}\left(\mathbb{S}^{n-1} \times[0,1[)\right.$ is just the degree of the restriction $\omega_{\mid \mathbb{S}^{n-1} \times\{0\}}$, where the degree of 0 is $-\infty$. 
(d) Given a tubular neighborhood $\tau: T \rightarrow S$ (cf. 2.3) we know that we can identify $\Pi_{\mathcal{F}}^{*}(T)$ with $\Pi_{\mathcal{F} \times \mathcal{I}}^{*}(D \times[0,1[)$ through $\nabla$ (cf. 3.1.1(e)). Since $\nabla: D \times] 0,1[\rightarrow T-S$ is a $K$-diffeomorphism, the family of strata of $T$ is $\left\{\nabla\left(S^{\prime} \times\right] 0,1[) \mid S^{\prime}\right.$ is a stratum of $\left.D\right\} \cup\{S\}$. So, for any $\omega \in \Pi_{\mathcal{F}}^{*}(T)$ we have:

- $\|\omega\|_{\nabla\left(S^{\prime} \times\right] 0,1[)}=\left\|\nabla^{*} \omega\right\|_{\left.S^{\prime} \times\right] 0,1[}$.

- $\|\omega\|_{S}=\left\|\left(\nabla^{*} \omega\right)_{\mid D \cap R_{\mathcal{F}}} \times\{0\}\right\|_{\tau}$, where $\|\alpha\|_{\tau}$ denotes the vertical degree of $\alpha \in \Omega^{*}\left(D \cap R_{\mathcal{F}}\right)$ relative to the projection $\tau:\left(D \cap R_{\mathcal{F}}\right) \times\{0\} \equiv$ $D \cap R_{\mathcal{F}} \rightarrow S$ (cf. Proposition 2.3.1).

(e) Let us illustrate this notion with the example of 3.1.1(f). The perverse forms are just the differential forms on $\mathbb{S}^{5} \times \mathbb{D}^{2}$. The perverse degree \|\|$_{\mathbb{S}^{1}}$ is measured relative to the trivial fibration $\mathbb{S}^{5} \times \mathbb{S}^{1} \rightarrow \mathbb{S}^{1}$. So, if $\theta_{5}$ is a volume form of $\mathbb{S}^{5}, \theta_{1}$ a volume form on $\mathbb{S}^{1}$ and $(x, y)$ the coordinates on $\mathbb{D}^{2}$, we have

$$
\left\|\theta_{1}\right\|_{\mathbb{S}^{1}}=0, \quad\left\|\theta_{5}\right\|_{\mathbb{S}^{1}}=5, \quad\left\|\theta_{5} \wedge(x d x+y d y)\right\|_{\mathbb{S}^{1}}=-\infty .
$$

(f) A perverse form with $\|\omega\|_{S} \leq 0$ and $\|d \omega\|_{S} \leq 0$ induces a differential form $\omega_{S}$ on $S$. When this happens for each stratum $S$ we conclude that $\omega \equiv\left\{\omega_{S}\right\}$ is a Verona controlled form (cf. [19]).

Proof. Let $\left(U_{2}, \varphi_{2}\right)$ be a conical chart of a point of a singular stratum $S$. The conditions $\|\omega\|_{S} \leq 0$ and $\|d \omega\|_{S} \leq 0$ give the existence of a form $\eta_{\varphi_{2}} \in \Omega^{*}\left(U_{2} \cap S\right)$ with $\omega_{\varphi_{2}}=P_{\varphi_{2}}^{*} \eta_{\varphi_{2}}$ on $\mathbb{R}^{m-n} \times R_{\mathcal{G}} \times\{0\}$. Let $\left(U_{1}, \varphi_{1}\right)$ be another conical chart of a point of $S$ with $\left(U_{1}, \varphi_{1}\right) \subset\left(U_{2}, \varphi_{2}\right)$. From Proposition 1.3.1 we have

$$
P_{\varphi_{1}}^{*} \eta_{\varphi_{1}}=\omega_{\varphi_{1}}=\Phi_{1,2}^{*} \omega_{\varphi_{2}}=\Phi_{1,2}^{*} P_{\varphi_{2}}^{*} \eta_{\varphi_{2}}=P_{\varphi_{1}}^{*} \eta_{\varphi_{2}}
$$

and therefore $\eta_{\varphi_{1}}=\eta_{\varphi_{2}}$ on $U_{1} \cap S$. This implies that the forms $\left\{\eta_{\varphi_{i}}\right\}$ match up to define a differential form $\omega_{S} \in \Omega^{*}(S)$.

(g) An open foliated embedding $f:\left(M_{1}, \mathcal{F}_{1}\right) \rightarrow\left(M_{2}, \mathcal{F}_{2}\right)$ between two foliated conical manifolds induces a dgca operator $f^{*}: \Pi_{\mathcal{F}_{2}}^{*}\left(M_{2}\right) \rightarrow \Pi_{\mathcal{F}_{1}}^{*}\left(M_{1}\right)$ which preserves the perverse degree (cf. Proposition 1.3.1).

3.3. Basic cohomology. The basic cohomology of a foliation $\mathcal{F}$ is an important tool in the study of its transversal structure and plays the role of the cohomology of the leaf space $M / \mathcal{F}$, which can be a wild topological space.

Let $(M, \mathcal{F})$ be a foliated manifold. A differential form $\omega \in \Omega^{*}(M)$ is basic if

$$
i_{X} \omega=i_{X} d \omega=0
$$

for each vector field $X$ on $M$ tangent to the foliation $\mathcal{F}$. For example, a function $f$ is basic iff $f$ is constant on the leaves. We denote by $\Omega^{*}(M / \mathcal{F})$ 
the complex of basic forms. Since sums and products of basic forms are basic, the complex of basic forms is a dgca. Its cohomology $H^{*}(M / \mathcal{F})$ is the basic cohomology of $(M, \mathcal{F})$. We also use the relative basic cohomology $H^{*}((M, F) / \mathcal{F})$, that is, the cohomology computed from the complex of basic forms vanishing on the saturated set $F$.

When the foliation comes from a fibration $f: M \rightarrow B$ with connected fibers, the leaf space $M / \mathcal{F}$ is in fact the manifold $B$ and the basic cohomology $H^{*}(M / \mathcal{F})$ is the cohomology of $B$.

\subsubsection{REMARKS.}

(a) The basic cohomology does not use the stratification $S_{\mathcal{F}}$.

(b) Let us illustrate this notion with an example. Consider the isometric action $\Psi: \mathbb{R} \times \mathbb{S}^{5} \rightarrow \mathbb{S}^{5}$ defined by $\Psi\left(t,\left(z_{0}, z_{1}, z_{2}\right)\right)=\left(e^{a \pi i t} z_{0}, e^{b \pi i t} z_{1}, e^{c \pi i t} z_{2}\right)$ with $(a, b, c) \neq(0,0,0)$. The induced foliation $\mathcal{G}$ is regular and $H^{i}\left(\mathbb{S}^{5} / \mathcal{G}\right)$ is the following:

\begin{tabular}{|c|c|c|c|c|}
\hline$i=0$ & $i=1$ & $i=2$ & $i=3$ & $i=4$ \\
\hline $\mathbb{R}$ & 0 & $\mathbb{R} \cdot[e]$ & 0 & $\mathbb{R} \cdot\left[e^{2}\right]$ \\
\hline
\end{tabular}

Here, the cycle $e \in \Omega^{2}\left(\mathbb{S}^{5} / \mathcal{G}\right)$ is an Euler form. Notice that this cohomology is finite-dimensional and satisfies the Poincaré duality. These facts are true for any regular isometric flow on a compact manifold (see [6] and [7]).

Consider now the singular isometric flow defined in 3.1.1(f). Then $H^{i}\left(\mathbb{S}^{7} / \mathcal{F}\right)$ is

\begin{tabular}{|c|c|c|c|c|c|c|}
\hline$i=0$ & $i=1$ & $i=2$ & $i=3$ & $i=4$ & $i=5$ & $i=6$ \\
\hline $\mathbb{R}$ & 0 & 0 & 0 & $\mathbb{R} \cdot[\beta \wedge e]$ & 0 & $\mathbb{R} \cdot\left[\beta \wedge e^{2}\right]$ \\
\hline
\end{tabular}

The cycle $\beta \in \Omega^{2}\left(\mathbb{S}^{7} / \mathcal{F}\right)$ is the form induced by 1 from the double suspension $\mathbb{S}^{7} \equiv \Sigma \Sigma \mathbb{S}^{5}$.

Notice that this cohomology is finite-dimensional. This is true for any isometric flow on a compact manifold (see [21], [13]). On the other hand, the Poincaré duality is lost. We introduce the basic intersection cohomology in order to recover this property.

(c) A smooth foliated map $f:\left(M_{1}, \mathcal{F}_{1}\right) \rightarrow\left(M_{2}, \mathcal{F}_{2}\right)$ induces a dgca operator $f^{*}: \Omega^{*}\left(M_{2} / \mathcal{F}_{2}\right) \rightarrow \Omega^{*}\left(M_{1} / \mathcal{F}_{1}\right)$.

3.4. Basic intersection cohomology. The stratification $S_{\mathcal{F}}$ induced by a conical foliation $\mathcal{F}$ is rich enough to support an intersection cohomology theory.

Let $(M, \mathcal{F})$ be a conical foliated manifold. A perversity is any map $\bar{p}$ : $\mathrm{S}_{\mathcal{F}}^{\sigma} \rightarrow \mathbb{Z}$, where $\mathrm{S}_{\mathcal{F}}^{\sigma}$ is the family of singular strata. There are two particular perversities we are going to use: 
- the constant perversity $\bar{i}$ defined by $\bar{i}(S)=i$, where $i \in \mathbb{Z}$, and

- the (basic) top perversity $\bar{t}$ defined by

$$
\bar{t}(S)=\operatorname{codim}_{M} \mathcal{F}-\operatorname{codim}_{S} \mathcal{F}_{S}-2=\operatorname{codim}_{M} S-\operatorname{dim} \mathcal{G}_{S}-2
$$

Any two perversities can be added.

Associated to an open foliated embedding $f:\left(M^{\prime}, \mathcal{F}^{\prime}\right) \rightarrow(M, \mathcal{F})$ and a perversity $\bar{p}$ on $(M, \mathcal{F})$ there exists a perversity on $\left(M^{\prime}, \mathcal{F}^{\prime}\right)$, still written $\bar{p}$, defined by $\bar{p}\left(S^{\prime}\right)=\bar{p}(S)$ where $S^{\prime} \in \mathrm{S}_{\mathcal{F}^{\prime}}^{\sigma}$ and $S \in \mathrm{S}_{\mathcal{F}}^{\sigma}$ with $f\left(S^{\prime}\right) \subset S$.

The basic intersection cohomology appears when one considers basic forms whose perverse degree is controlled by a given perversity. We let

$$
\begin{aligned}
& \Omega_{\bar{p}}^{*}(M / \mathcal{F}) \\
& \quad=\left\{\omega \in \Omega^{*}\left(R_{\mathcal{F}} / \mathcal{F}_{R_{\mathcal{F}}}\right) \cap \Pi_{\mathcal{F}}^{*}(M) \mid \max \left(\|\omega\|_{S},\|d \omega\|_{S}\right) \leq \bar{p}(S) \forall S \in \mathrm{S}_{\mathcal{F}}^{\sigma}\right\}
\end{aligned}
$$

be the complex of basic perverse forms whose perverse degree (and that of their differential) is bounded by the perversity $\bar{p}$. It is a differential complex, but it is not an algebra, in fact the wedge product acts in the following way:

$$
\wedge: \Omega_{\bar{p}}^{i}(M / \mathcal{F}) \times \Omega_{\bar{q}}^{j}(M / \mathcal{F}) \rightarrow \Omega_{\bar{p}+\bar{q}}^{i+j}(M / \mathcal{F})
$$

(see $(13))$. The cohomology $\mathbb{H}_{\bar{p}}^{*}(M / \mathcal{F})$ of this complex is the basic intersection cohomology of $(M, \mathcal{F})$, or BIC for short, relative to the perversity $\bar{p}$ (cf. [15]).

\subsubsection{REMARKS.}

(a) The basic intersection cohomology coincides with the basic cohomology when the foliation $\mathcal{F}$ is regular, that is, when $\operatorname{depth} S_{\mathcal{F}}=0$. But it also generalizes the intersection cohomology of Goresky-MacPherson (cf. [8]) when the leaf space $B$ lies in the right category, that of stratified pseudomanifolds (cf. [15]).

(b) The perverse degree of a perverse form satisfies (14). But when this form is also basic we have

$$
\|\omega\|_{S} \leq \operatorname{dim} R_{\mathcal{G}}-\operatorname{dim} \mathcal{F}_{R_{\mathcal{G}}}=\operatorname{codim}_{M} \mathcal{F}-\operatorname{codim}_{S} \mathcal{F}_{S}-1=(\bar{t}+\overline{1})(S)
$$

(c) If $\omega \in \Omega_{\bar{t}}^{\ell-1}(M / \mathcal{F})$ and $\varphi:\left(\mathbb{R}^{m-n} \times c \mathbb{S}^{n-1}, \mathcal{H} \times c \mathcal{G}\right) \rightarrow(U, \mathcal{F})$ is a conical chart then

$$
\omega_{\varphi} \equiv 0 \quad \text { on } \mathbb{R}^{m-n} \times R_{\mathcal{G}} \times\{0\},
$$

where $\ell=\operatorname{codim}_{M} \mathcal{F}$.

Proof. Let $S$ be the stratum of $S_{\mathcal{F}}$ containing $\varphi\left(\mathbb{R}^{m-n} \times\{\vartheta\}\right)$. Notice that $\bar{t}(S)=\ell-((m-n)-\operatorname{dim} \mathcal{H})-2=n-\operatorname{dim} \mathcal{G}-2$. If the above assertion is not true then there exist $(x, z) \in \mathbb{R}^{m-n} \times R_{\mathcal{G}},\left\{v_{1}, \ldots, v_{m-n-\operatorname{dim} \mathcal{H}}\right\} \subset T_{x} \mathbb{R}^{m-n}$ and $\left\{w_{1}, \ldots, w_{n-1-\operatorname{dim} \mathcal{G}}\right\} \subset T_{z} \mathbb{S}^{n-1}$ with

$$
\omega(x, z, 0)\left(v_{1}, \ldots, v_{m-n-\operatorname{dim} \mathcal{H}}, w_{1}, \ldots, w_{n-1-\operatorname{dim} \mathcal{G}}\right) \neq 0 .
$$




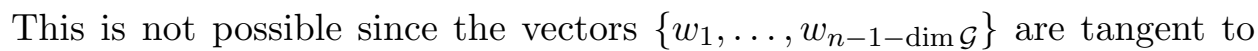
the fibers of $P_{\varphi}$ and $n-1-\operatorname{dim} \mathcal{G}>\bar{t}(S)$.

(d) Consider a tubular neighborhood $\tau: T \rightarrow S$ (cf. 2.3) and a perversity $\bar{p}$ on $T$. From 3.2.1(d) we see that $\nabla^{*}$ establishes an isomorphism between $\Omega_{\bar{p}}^{*}(T / \mathcal{F})$ and

$$
\left\{\omega \in \Omega _ { \overline { p } } ^ { * } \left(D \times\left[0,1[/ \mathcal{F} \times \mathcal{I}) \mid\left\|\omega_{\mid D \cap R_{\mathcal{F}}}\right\|_{\tau} \leq \bar{p}(S) \text { and }\left\|d \omega_{\mid D \cap R_{\mathcal{F}}}\right\|_{\tau} \leq \bar{p}(S)\right\} .\right.\right.
$$

Here, the perversity $\bar{p}$ on $D \times\left[0,1\left[\right.\right.$ is defined by $\bar{p}\left(S^{\prime} \times\left[0,1[)=\bar{p}\left(\nabla\left(S^{\prime} \times\right.\right.\right.\right.$ ] $0,1[))$.

(e) Let us illustrate this notion with the example of 3.1.1(f) (see also 3.3.1(b)). A direct calculation shows that $H_{\bar{p}}^{i}\left(\mathbb{S}^{7} / \mathcal{F}\right)$ is

\begin{tabular}{|c|c|c|c|c|c|c|c|}
\hline & $i=0$ & $i=1$ & $i=2$ & $i=3$ & $i=4$ & $i=5$ & $i=6$ \\
\hline $\bar{p} \leq \overline{-1}$ & 0 & 0 & $\mathbb{R} \cdot[\beta]$ & 0 & $\mathbb{R} \cdot[\beta \wedge e]$ & 0 & $\mathbb{R} \cdot\left[\beta \wedge e^{2}\right]$ \\
\hline $\bar{p}=\overline{0}$ & $\mathbb{R}$ & 0 & 0 & 0 & $\mathbb{R} \cdot[\beta \wedge e]$ & 0 & $\mathbb{R} \cdot\left[\beta \wedge e^{2}\right]$ \\
\hline$\cdots$ & $\cdots$ & $\cdots$ & $\cdots$ & $\cdots$ & $\cdots$ & $\cdots$ & $\cdots$ \\
\hline $\bar{p}=\bar{t}$ & $\mathbb{R}$ & 0 & $\mathbb{R} \cdot[e]$ & 0 & $\mathbb{R} \cdot[\beta \wedge e]$ & 0 & 0 \\
\hline $\bar{p} \geq \bar{t}+\overline{1}$ & $\mathbb{R}$ & 0 & $\mathbb{R} \cdot[e]$ & 0 & $\mathbb{R} \cdot\left[e^{2}\right]$ & 0 & 0 \\
\hline
\end{tabular}

The first line is the relative basic cohomology $H^{*}\left(\left(\mathbb{S}^{7}, \mathbb{S}^{1}\right) / \mathcal{F}\right)$, the second line is the basic cohomology $H^{*}\left(\mathbb{S}^{7} / \mathcal{F}\right)$ and the last line is the basic cohomology $H^{*}\left(\left(\mathbb{S}^{7}-\mathbb{S}^{1}\right) / \mathcal{F}\right)$. These cohomologies are finite-dimensional and satisfy the following Poincaré duality:

$$
I_{\bar{p}}^{i}(M / \mathcal{F}) \cong H_{\bar{q}}^{j}(M / \mathcal{F})
$$

for $i+j=6$ and $\bar{p}+\bar{q}=\bar{t}$.

We shall prove that all these facts are true for any abelian isometric flow on a compact manifold.

(f) Given an open foliated embedding $f:\left(M_{1}, \mathcal{F}_{1}\right) \rightarrow\left(M_{2}, \mathcal{F}_{2}\right)$ we have the induced differential operator $f^{*}: \Omega_{\bar{p}}^{*}\left(M_{2} / \mathcal{F}_{2}\right) \rightarrow \Omega_{\bar{p}}^{*}\left(M_{1} / \mathcal{F}_{1}\right)(\mathrm{cf} .3 .1 .1(\mathrm{~h})$ and 3.3.1(c)). If $f$ is a foliated diffeomorphism then $f^{*}$ is a differential isomorphism.

We now present some of the technical tools used in this work. We fix a conical foliated manifold $(M, \mathcal{F})$ and a perversity $\bar{p}$.

3.5. Local calculations. The intersection basic cohomology, like the basic cohomology, is not easily computable. It becomes computable for singular foliations defined by an abelian isometric group, as will be observed in the next section. Nevertheless, the typical calculations for the BIC are classical. The following two propositions were announced in [15] without proof. 
3.5.1. Proposition. Let $\left(\mathbb{R}^{k}, \mathcal{H}\right)$ be a simple foliation. Let $\bar{p}$ be the perversity defined on the conical foliated manifold $\left(\mathbb{R}^{k} \times M, \mathcal{H} \times \mathcal{F}\right)$ by $\bar{p}\left(\mathbb{R}^{k} \times S\right)=\bar{p}(S)$. The canonical projection $\mathrm{pr}: \mathbb{R}^{k} \times M \rightarrow M$ induces an isomorphism

$$
\mathbb{H}_{\bar{p}}^{*}(M / \mathcal{F}) \cong \mathbb{H}_{\bar{p}}^{*}\left(\mathbb{R}^{k} \times M / \mathcal{H} \times \mathcal{F}\right) .
$$

Proof. Fix a base point $a \in \mathbb{R}^{k}$ and let $\iota: M \rightarrow \mathbb{R}^{k} \times M$ be the inclusion defined by $\iota(x)=(a, x)$. Notice that a conical chart $(U, \varphi)$ on $M$ induces the conical chart $\left(\mathbb{R}^{k} \times U, \operatorname{Id}_{\mathbb{R}^{k}} \times \varphi\right)$ on $\mathbb{R}^{k} \times M$. Under these charts the projection pr becomes the canonical projection $\mathbb{R}^{k} \times \mathbb{R}^{m-n} \times c \mathbb{S}^{n-1} \rightarrow \mathbb{R}^{m-n} \times c \mathbb{S}^{n-1}$ defined by $(u, v, \zeta) \mapsto(v, \zeta)$. The inclusion $\iota$ becomes the inclusion $\mathbb{R}^{m-n} \times$ $c \mathbb{S}^{n-1} \rightarrow \mathbb{R}^{k} \times \mathbb{R}^{m-n} \times c \mathbb{S}^{n-1}$ defined by $(v, \zeta) \mapsto(a, v, \zeta)$. An inductive argument on the depth shows that $\mathrm{pr}^{*}: \Omega_{\bar{p}}^{*}(M / \mathcal{F}) \rightarrow \Omega_{\bar{p}}^{*}\left(\mathbb{R}^{k} \times M / \mathcal{H} \times \mathcal{F}\right)$ and $\iota^{*}: \Omega_{\bar{p}}^{*}\left(\mathbb{R}^{k} \times M / \mathcal{H} \times \mathcal{F}\right) \rightarrow \Omega_{\bar{p}}^{*}(M / \mathcal{F})$ are well defined differential operators.

Since $\iota^{*} \circ \mathrm{pr}^{*}$ is the identity, it suffices to prove that $\mathrm{pr}^{*} \circ \iota^{*}$ is homotopic to the identity.

The foliated homotopy $k_{0}: \mathbb{R}^{k} \times[0,1] \rightarrow \mathbb{R}^{k}$ defined by $k_{0}(u, t)=t u$ induces the homotopy $k_{1}: \mathbb{R}^{k} \times M \times[0,1] \rightarrow \mathbb{R}^{k} \times M$ defined by $k_{1}(u, x, t)=$ $\left(k_{0}(u, t), x\right)$. This homotopy does not involve the $M$-factor, so it induces a morphism $k_{1}^{*}: \Pi_{\mathcal{H} \times \mathcal{F}}^{*}\left(\mathbb{R}^{k} \times M\right) \rightarrow \Pi_{\mathcal{H} \times \mathcal{F}}^{*}\left(\mathbb{R}^{k} \times M \times[0,1]\right)$ which preserves the perverse degree. We also have $d \circ k_{1}^{*}=k_{1}^{*} \circ d$ and therefore the differential morphism

$$
k_{1}^{*}: \Omega_{\bar{p}}^{*}\left(\mathbb{R}^{k} \times M\right) \rightarrow \Omega_{\bar{p}}^{*}\left(\mathbb{R}^{k} \times M \times[0,1]\right) .
$$

Integration along the $[0,1]$-factor does not involve $M$. So, the operator $K: \Pi_{\mathcal{H} \times \mathcal{F}}^{*}\left(\mathbb{R}^{k} \times M\right) \rightarrow \Pi_{\mathcal{H} \times \mathcal{F}}^{*-1}\left(\mathbb{R}^{k} \times M\right)$ given by $K \omega=\int_{0}^{1} k_{1}^{*} \omega$ is well defined and preserves the perverse degree. On the other hand, it satisfies the homotopy equality

$$
d \circ K+K \circ d=\operatorname{pr}^{*} \circ \iota^{*}-\mathrm{Id} .
$$

This implies that $d K$ also preserves the perverse degree. We conclude that

$$
K: \Omega_{\bar{p}}^{*}\left(\mathbb{R}^{k} \times M / \mathcal{H} \times \mathcal{F}\right) \rightarrow \Omega_{\bar{p}}^{*-1}\left(\mathbb{R}^{k} \times M / \mathcal{H} \times \mathcal{F}\right)
$$

is well defined and is a homotopy operator between $\operatorname{pr}^{*} \circ \iota^{*}$ and the identity.

For the cone $\left(c \mathbb{S}^{n-1}, c \mathcal{G}\right)$ (cf. 1.1.1) we have:

3.5.2. Proposition. Let $\mathcal{G}$ be a conical foliation without 0-dimensional leaves on the sphere $\mathbb{S}^{n-1}$. A perversity $\bar{p}$ on $c \mathbb{S}^{n-1}$ gives the perversity $\bar{p}$ on $\mathbb{S}^{n-1}$ defined by $\bar{p}(S)=\bar{p}(S \times] 0,1[)$. The canonical projection $\chi: \mathbb{S}^{n-1} \times$ ] $0,1\left[\rightarrow \mathbb{S}^{n-1}\right.$ induces an isomorphism

$$
\mathbb{H}_{\bar{p}}^{i}\left(c \mathbb{S}^{n-1} / c \mathcal{G}\right)= \begin{cases}\mathbb{H}_{\bar{p}}^{i}\left(\mathbb{S}^{n-1} / \mathcal{G}\right) & \text { if } i \leq \bar{p}(\{\vartheta\}), \\ 0 & \text { if } i>\bar{p}(\{\vartheta\})\end{cases}
$$


Proof. The statement about perversities is clear. From 3.2.1(c) we have

$$
\Omega_{\bar{p}}^{j}\left(c \mathbb{S}^{n-1} / c \mathcal{G}\right)= \begin{cases}\Omega_{\bar{p}}^{j}\left(\mathbb{S}^{n-1} \times[0,1[/ \mathcal{G} \times \mathcal{I})\right. & \text { if } j<\bar{p}(\{\vartheta\}), \\ \Omega_{\bar{p}}^{j}\left(\mathbb{S}^{n-1} \times\left[0,1[/ \mathcal{G} \times \mathcal{I}) \cap d^{-1} \operatorname{Ker} \iota^{*}\right.\right. & \text { if } j=\bar{p}(\{\vartheta\}), \\ \Omega_{\bar{p}}^{j}\left(\mathbb{S}^{n-1} \times\left[0,1[/ \mathcal{G} \times \mathcal{I}) \cap \operatorname{Ker} \iota^{*}\right.\right. & \text { if } j>\bar{p}(\{\vartheta\}) .\end{cases}
$$

Here $\iota: \mathbb{S}^{n-1} \rightarrow \mathbb{S}^{n-1} \times[0,1[$ is the inclusion defined by $\iota(\theta)=(\theta, 0)$. Proceeding as in the above proposition we get the desired isomorphism.

3.6. Mayer-Vietoris. An open covering $\{U, V\}$ of $M$ by saturated open subsets is basic if there exists a subordinate partition of unity made up of controlled basic functions. Basic coverings may or may not exist. For such a covering we have the Mayer-Vietoris sequence

$$
0 \rightarrow \Omega_{\bar{p}}^{*}(M / \mathcal{F}) \rightarrow \Omega_{\bar{p}}^{*}(U / \mathcal{F}) \oplus \Omega_{\bar{p}}^{*}(V / \mathcal{F}) \rightarrow \Omega_{\bar{p}}^{*}((U \cap V) / \mathcal{F}) \rightarrow 0,
$$

where the maps are defined by $\omega \mapsto(\omega, \omega)$ and $(\alpha, \beta) \mapsto \alpha-\beta$. The third map is onto since the elements of the partition of unity are controlled basic functions. Thus, the sequence is exact. This result is no longer true for more general coverings.

The Mayer-Vietoris sequence allows us to make computations when the conical foliated manifold has a suitable finite covering. The passage from the finite case to the general case may be done by using an adapted version of Bredon's trick of [4, p. 289]:

3.6.1. BREDON's TRICK. Let $X$ be a paracompact topological space (resp. compact topological space) and let $\left\{U_{\alpha}\right\}$ be an open covering, closed under finite intersections. Suppose that $Q(U)$ is a statement about open subsets of $X$, satisfying the following three properties:

(BT1) $Q\left(U_{\alpha}\right)$ is true for each $\alpha$;

(BT2) $Q(U) \& Q(V) \& Q(U \cap V) \Rightarrow Q(U \cup V)$ for any open subsets $U$ and $V$ of $X$;

(BT3) $Q\left(U_{i}\right) \Rightarrow Q\left(\bigcup_{i} U_{i}\right)$ for any disjoint (resp. finite disjoint) family $\left\{U_{i}\right\}$ of open subsets of $X$.

Then $Q(X)$ is true.

3.7. Compact supports. For the study of Poincaré duality we shall need the notion of cohomology with compact supports. We define the support of a perverse form $\omega \in \Pi_{\mathcal{F}}^{*}(M)$ as the closure (in $M$ !)

$$
\operatorname{supp} \omega=\overline{\left\{x \in M-\Sigma_{\mathcal{F}} \mid \omega(x) \neq 0\right\}} \text {. }
$$

We have $\operatorname{supp}\left(\omega+\omega^{\prime}\right) \subset \operatorname{supp} \omega \cup \operatorname{supp} \omega^{\prime}, \operatorname{supp}\left(\omega \wedge \omega^{\prime}\right) \subset \operatorname{supp} \omega \cap \operatorname{supp} \omega^{\prime}$ and supp $d \omega \subset \operatorname{supp} \omega$. We define

$$
\Omega_{\bar{p}, \mathrm{c}}^{*}(M / \mathcal{F})=\left\{\omega \in \Omega_{\bar{p}}^{*}(M / \mathcal{F}) \mid \operatorname{supp} \omega \text { is compact }\right\} \text {. }
$$


It is a differential complex, but not an algebra; in fact, the wedge product acts in this way:

$$
\wedge: \Omega_{\bar{p}, \mathrm{c}}^{i}(M / \mathcal{F}) \times \Omega_{\bar{q}}^{j}(M / \mathcal{F}) \rightarrow \Omega_{\bar{p}+\bar{q}, \mathrm{c}}^{i+j}(M / \mathcal{F})
$$

(see $(13))$. The cohomology $\mathbb{H}_{\bar{p}, \mathrm{c}}^{*}(M / \mathcal{F})$ of this complex is the basic intersection cohomology with compact supports of $(M, \mathcal{F})$, relative to the perversity $\bar{p}$. Of course, when $M$ is compact we have $\mathbb{H}_{\bar{p}, \mathrm{c}}^{*}(M / \mathcal{F})=\mathbb{H}_{\bar{p}}^{*}(M / \mathcal{F})$.

For $\Sigma_{\mathcal{F}}=\emptyset$, this notion generalizes the basic cohomology with compact supports.

Given a basic covering $\{U, V\}$ of $M$ we have the Mayer-Vietoris sequence

$$
0 \rightarrow \Omega_{\bar{p}, \mathrm{c}}^{*}((U \cap V) / \mathcal{F}) \rightarrow \Omega_{\bar{p}, \mathrm{c}}^{*}\left(U / \mathcal{F}_{U}\right) \oplus \Omega_{\bar{p}, \mathrm{c}}^{*}\left(V / \mathcal{F}_{V}\right) \rightarrow \Omega_{\bar{p}, \mathrm{c}}^{*}(M / \mathcal{F}) \rightarrow 0,
$$

where the maps are defined by $\omega \mapsto(\omega, \omega)$ and $(\alpha, \beta) \mapsto \alpha-\beta$. The third map is onto since the elements of the partition of unity are controlled basic functions. Thus, the sequence is exact.

We now give some local calculations. Given a simple foliation $\left(\mathbb{R}^{k}, \mathcal{H}\right) \equiv$ $\left(\mathbb{R}^{a} \times \mathbb{R}^{b}, \mathcal{J} \times \mathcal{I}\right)$, where $\mathcal{J}$ is the one-leaf foliation of $\mathbb{R}^{a}$ and $\mathcal{I}$ the point foliation of $\mathbb{R}^{b}$, we have $H_{\mathrm{c}}^{*}\left(\mathbb{R}^{k} / \mathcal{H}\right)=\mathbb{R}$ generated by $\left[f d x_{1} \wedge \cdots \wedge d x_{b}\right]$ where $f$ is a bump function: $f \in \mathcal{C}^{\infty}\left(\mathbb{R}^{b}\right)$ with $\int_{\mathbb{R}^{b}} f=1$ and compact support.

3.7.1. Proposition. Let $\left(\mathbb{R}^{k}, \mathcal{H}\right)$ be a simple foliation. We have an isomorphism

$$
\mathbb{H}_{\bar{p}, \mathrm{c}}^{*}(M / \mathcal{F}) \cong H_{\bar{p}, \mathrm{c}}^{*+b}\left(\mathbb{R}^{k} \times M / \mathcal{H} \times \mathcal{F}\right)
$$

given by $[\beta] \mapsto\left[f d x_{1} \wedge \cdots \wedge d x_{b} \wedge \beta\right]$, where $f \in \mathcal{C}^{\infty}\left(\mathbb{R}^{b}\right)$ is a bump function.

Proof. Notice first that $\Omega_{\bar{p}, \mathrm{c}}^{*}\left(\mathbb{R}^{k} \times M / \mathcal{H} \times \mathcal{F}\right)=\Omega_{\bar{p}, \mathrm{c}}^{*}\left(\mathbb{R}^{b} \times M / \mathcal{I} \times \mathcal{F}\right)$. It suffices to deal with the case $b=1$.

Let us introduce some notation. Suppose $\beta \in \Omega^{*}\left(R_{\mathcal{F}} \times M\right)$ does not include the $d t$ factor. We denote by $\int_{-}^{c} \beta(s) \wedge d s$ and $\int_{c}^{-} \beta(s) \wedge d s$ the forms in $\Omega^{*}\left(R_{\mathcal{F}} \times M\right)$ obtained from $\beta$ by integration with respect to $s$ :

$$
\begin{aligned}
& \left(\int_{-}^{c} \beta(s) \wedge d s\right)(x, t)\left(\vec{v}_{1}, \ldots, \vec{v}_{i}\right)=\int_{t}^{c} \beta(x, s)\left(\vec{v}_{1}, \ldots, \vec{v}_{i}\right) d s \\
& \left.\left(\int_{c}^{-} \beta(s) \wedge d s\right)(x, t)\left(\vec{v}_{1}, \ldots, \vec{v}_{i}\right)\right)=\int_{c}^{t} \beta(x, s)\left(\vec{v}_{1}, \ldots, \vec{v}_{i}\right) d s
\end{aligned}
$$

where $c \in] a, b\left[,(x, t) \in R_{\mathcal{F}} \times\right] a, b\left[\right.$ and $\left(\vec{v}_{1}, \ldots, \vec{v}_{i}\right) \in T_{(x, t)}\left(R_{\mathcal{F}} \times\right] a, b[)$.

A general $\omega \in \Omega_{\bar{p}, \mathrm{c}}^{*}(\mathbb{R} \times M / \mathcal{I} \times \mathcal{F})$ is of the form

$$
\omega=\alpha+\beta \wedge d t
$$

where $\alpha, \beta \in \Omega_{\bar{p}, \mathrm{c}}^{*}(\mathbb{R} \times M / \mathcal{I} \times \mathcal{F})$ do not contain $d t$. Consider the differential 
operators

$$
\begin{aligned}
& \Delta: \Omega_{\bar{p}, \mathrm{c}}^{*}(M / \mathcal{F}) \rightarrow \Omega_{\bar{p}, \mathrm{c}}^{*-1}(\mathbb{R} \times M / \mathcal{I} \times \mathcal{F}), \\
& \nabla: \Omega_{\bar{p}, \mathrm{c}}^{*}(\mathbb{R} \times M / \mathcal{I} \times \mathcal{F}) \rightarrow \Omega_{\bar{p}, \mathrm{c}}^{*+1}(M / \mathcal{F}),
\end{aligned}
$$

defined by

$$
\Delta(\alpha+\beta \wedge d t)=\int_{-\infty}^{\infty} \beta(s) \wedge d s \quad \text { and } \quad \nabla(\beta)=f d t \wedge \beta .
$$

Notice that $\Delta \circ \nabla=\operatorname{Id}\left(\right.$ up to sign), which gives $\nabla^{*} \circ \Delta^{*}=\operatorname{Id}$ (up to sign). We now prove $\Delta^{*} \circ \nabla^{*}=\mathrm{Id}$. Consider $[\omega=\alpha+\beta \wedge d t] \in \mathbb{H}_{\bar{p}, \mathrm{c}}^{*}(\mathbb{R} \times M / \mathcal{I} \times \mathcal{F})$. Define $\eta \in \Omega_{\bar{p}, \mathrm{c}}^{*-1}(\mathbb{R} \times M / \mathcal{I} \times \mathcal{F})$ by

$$
\eta=\left(\int_{-\infty}^{t} f(s) d s\right)\left(\int_{t}^{\infty} \beta(s) \wedge d s\right)-\left(\int_{t}^{\infty} f(s) d s\right)\left(\int_{-\infty}^{t} \beta(s) \wedge d s\right) .
$$

A straightforward calculation gives

$$
d \eta=f d t \wedge \int_{-\infty}^{\infty} \beta(s) \wedge d s-\beta \wedge d t-\alpha=\Delta^{*} \circ \nabla^{*}(\omega)-\omega .
$$

This ends the proof.

For the computation of $\mathbb{H}_{\bar{p}}^{*}\left(c \mathbb{S}^{n-1} / c \mathcal{G}\right)$ we consider $g \in \mathcal{C}^{\infty}([0,1[)$ with $g \equiv 1$ on $[0,1 / 4], g \equiv 0$ on $\left[3 / 4,1\left[\right.\right.$ and $\int_{0}^{1} g=1$.

3.7.2. Proposition. Let $\mathcal{G}$ be a conical foliation without 0-dimensional leaves on the sphere $\mathbb{S}^{n-1}$. We have an isomorphism

$$
\mathbb{H}_{\bar{p}, \mathrm{c}}^{i}\left(c \mathbb{S}^{n-1} / c \mathcal{G}\right)= \begin{cases}0 & \text { if } i \leq \bar{p}(\{\vartheta\})+1, \\ \mathbb{H}_{\bar{p}}^{i-1}\left(\mathbb{S}^{n-1} / \mathcal{G}\right) & \text { if } i \geq \bar{p}(\{\vartheta\})+2\end{cases}
$$

given by $[g d t \wedge \omega] \leftarrow[\omega]$.

Proof. From 3.2.1(c) we have

$$
\Omega_{\bar{p}, \mathrm{c}}^{i}\left(c \mathbb{S}^{n-1} / c \mathcal{G}\right)= \begin{cases}\Omega_{\bar{p}, \mathrm{c}}^{i}\left(\mathbb{S}^{n-1} \times[0,1[/ \mathcal{G} \times \mathcal{I})\right. & \text { if } i<\bar{p}(\{\vartheta\}), \\ \Omega_{\bar{p}, \mathrm{c}}^{i}\left(\mathbb{S}^{n-1} \times\left[0,1[/ \mathcal{G} \times \mathcal{I}) \cap d^{-1} \operatorname{Ker} \iota^{*}\right.\right. & \text { if } i=\bar{p}(\{\vartheta\}), \\ \Omega_{\bar{p}, \mathrm{c}}^{i}\left(\mathbb{S}^{n-1} \times\left[0,1[/ \mathcal{G} \times \mathcal{I}) \cap \operatorname{Ker} \iota^{*}\right.\right. & \text { if } i>\bar{p}(\{\vartheta\}) .\end{cases}
$$

Here $\iota: \mathbb{S}^{n-1} \rightarrow \mathbb{S}^{n-1} \times[0,1[$ is the inclusion defined by $\iota(\theta)=(\theta, 0)$. Consider a cycle $\omega=\alpha+d t \wedge \beta \in \Omega_{\bar{p}, \mathrm{c}}^{i}\left(c \mathbb{S}^{n-1} / \mathcal{G}\right)$. Notice that

$$
\omega=d\left(\int_{-}^{1} \beta(s) \wedge d s\right)
$$

with $\left(\int_{-}^{1} \beta(s) \wedge d s\right) \in \Omega_{\bar{p}, \mathrm{c}}^{i-1}\left(\mathbb{S}^{n-1} \times\left[0,1[/ \mathcal{G} \times \mathcal{I})\right.\right.$. This gives $\mathbb{H}_{\bar{p}, \mathrm{c}}^{i}\left(c \mathbb{S}^{n-1} / c \mathcal{G}\right)$ $=0$ if $i \leq \bar{p}(\vartheta)+1$. Now, it suffices to prove that $[\omega] \mapsto[g d t \wedge \omega]$ establishes 
an isomorphism between $H_{\bar{p}}^{*-1}\left(\mathbb{S}^{n-1} / \mathcal{G}\right)$ and

$$
\begin{aligned}
H^{*}\left(\Omega _ { \overline { p } , \mathrm { c } } \left(\mathbb{S}^{n-1} \times[0,1[/ \mathcal{G} \times \mathcal{I})\right.\right. & \left.\cap \operatorname{Ker} \iota^{*}\right) \\
= & \mathbb{H}_{\bar{p}, \mathrm{c}}^{*}\left(\left(\mathbb{S}^{n-1} \times\left[0,1\left[, \mathbb{S}^{n-1} \times\{0\}\right) / \mathcal{G} \times \mathcal{I}\right) .\right.\right.
\end{aligned}
$$

The proof is exactly the same as that of the previous proposition, with $-\infty$ replaced by 0 .

3.8. Twisted product. In this section we show how to compute the BIC of the twisted product $K \times_{H} \mathbb{R}^{n}$ (cf. 2.2) in terms of the group $K$ and the slice $\mathbb{R}^{n}$.

Notice first that a perversity $\bar{p}$ on $\left(K \times \mathbb{R}^{n}, \mathcal{F}_{K} \times \mathcal{F}_{\mathbb{R}^{n}}\right)\left(\right.$ resp. $\left(K \times_{H}\right.$ $\left.\left.\mathbb{R}^{n}, \mathcal{F}_{\text {tw }}\right)\right)$ is determined by a perversity $\bar{p}$ on $\left(\mathbb{R}^{n}, \mathcal{F}_{\mathbb{R}^{n}}\right)$ by putting $\bar{p}(K \times S)=$ $\bar{p}(S)(\operatorname{resp} . \bar{p}(R(K \times S))=\bar{p}(S)$, see $(5))$.

3.8.1. Lemma. The natural projection $R: K \times \mathbb{R}^{n} \rightarrow K \times_{H} \mathbb{R}^{n}$ induces a differential monomorphism

$$
R^{*}: \Omega_{\bar{p}}^{*}\left(K \times_{H} \mathbb{R}^{n} / \mathcal{F}_{\text {tw }}\right) \rightarrow \Omega_{\bar{p}}^{*}\left(K \times \mathbb{R}^{n} / \mathcal{F}_{K} \times \mathcal{F}_{\mathbb{R}^{n}}\right)
$$

for any perversity $\bar{p}$.

Proof. We proceed in several steps.

(a) A foliated atlas for $\pi: K \rightarrow K / H$. Since $\pi$ is an $H$-principal bundle, it has an atlas $\mathcal{A}_{\#}=\left\{\varphi: \pi^{-1}(U) \rightarrow U \times H\right\}$ of $H$-equivariant foliated charts. $H$-equivariance means $\varphi(h \cdot k)=\left(\pi(k), h \cdot h_{0}\right)$ if $\varphi(k)=\left(\pi(k), h_{0}\right)$. We study the foliation $\varphi_{*} \mathcal{F}_{K}$. By equivariance, $\varphi_{*} X_{u}=\left(0, Z_{u}\right)$ for each $u \in \mathfrak{g} \cap \mathfrak{h}$. Thus, the trace of the foliation $\varphi_{*} \mathcal{F}_{K}$ on the fibers of the canonical projection pr: $U \times H \rightarrow U$ is $\mathcal{F}_{H}$. On the other hand, since $\pi$ is a $G$-equivariant submersion, we have $\pi_{*} \mathcal{F}_{K}=\mathcal{F}_{K / H}$, which gives $\operatorname{pr}_{*} \varphi_{*} \mathcal{F}_{K}=\mathcal{F}_{K / H}$. We conclude that $\varphi_{*} \mathcal{F}_{K} \subset \mathcal{F}_{K / H} \times \mathcal{F}_{H}$. For dimensional reasons we get $\varphi_{*} \mathcal{F}_{K}=$ $\mathcal{F}_{K / H} \times \mathcal{F}_{H}$. The atlas $\mathcal{A}_{\#}$ is an $H$-equivariant foliated atlas of $\pi$.

(b) A foliated atlas of $K \times_{H} \mathbb{R}^{n}$. We claim that $\mathcal{A}^{\#}=\left\{\bar{\varphi}: \pi^{-1}(U) \times_{H}\right.$ $\left.\mathbb{R}^{n} \rightarrow U \times \mathbb{R}^{n} \mid(U, \varphi) \in \mathcal{A}_{\#}\right\}$ is a foliated atlas of $K \times_{H} \mathbb{R}^{n}$ where the map $\bar{\varphi}$ is defined by $\bar{\varphi}(\langle k, z\rangle)=\left(\pi(k), \Theta\left(\left(\varphi^{-1}(\pi(k), e)\right)^{-1} \cdot k, z\right)\right)$. This map is a diffeomorphism whose inverse is $\bar{\varphi}^{-1}(u, z)=\left\langle\varphi^{-1}(u, e), z\right\rangle$. It satisfies

$$
\bar{\varphi}_{*} \mathcal{F}_{\mathrm{tw}} \stackrel{R \text {-equiv. }}{=} \bar{\varphi}_{*} R_{*}\left(\mathcal{F}_{K} \times \mathcal{I}\right)=\bar{\varphi}_{*} R_{*}\left(\varphi^{-1} \times \operatorname{Id}_{\mathbb{R}^{n}}\right)_{*}\left(\mathcal{F}_{K / H} \times \mathcal{F}_{H} \times \mathcal{I}\right) .
$$

A straightforward calculation shows $\bar{\varphi} \circ R \circ\left(\varphi^{-1} \times \operatorname{Id}_{\mathbb{R}^{n}}\right)=\operatorname{Id}_{U} \times \Theta$. Since $\mathcal{F}_{H}$ is defined by the action $\Gamma: G \cap H \times H \rightarrow H$, we have $\Theta_{*}\left(\mathcal{F}_{H} \times \mathcal{I}\right)=\mathcal{F}_{\mathbb{R}^{n}}$. Finally, $\bar{\varphi}_{*} \mathcal{F}_{\text {tw }}=\mathcal{F}_{K / H} \times \mathcal{F}_{\mathbb{R}^{n}}$. 
(c) Last step. Given $(U, \varphi) \in \mathcal{A}_{\#}$, we have the commutative diagram

$$
\begin{array}{ccc}
U \times H \times \mathbb{R}^{n} & \stackrel{\varphi^{-1} \times \mathrm{Id}_{\mathbb{R}^{d+1}}}{\longrightarrow} & K \times \mathbb{R}^{n} \\
P \downarrow & & R \downarrow \\
U \times \mathbb{R}^{n} & \stackrel{\bar{\varphi}^{-1}}{\longrightarrow} & K \times_{H} \mathbb{R}^{n}
\end{array}
$$

where $P(u, h, z)=(u, \Theta(h, z))$ and $R^{-1}\left(\operatorname{Im} \bar{\varphi}^{-1}\right)=\operatorname{Im} \varphi^{-1} \times \operatorname{Id}_{\mathbb{R}^{d+1}}$. We claim that

$$
R^{*}: \Omega_{\bar{p}}^{*}\left(K \times_{H} \mathbb{R}^{n} / \mathcal{F}_{\mathrm{tw}}\right) \rightarrow \Omega_{\bar{p}}^{*}\left(K \times \mathbb{R}^{n} / \mathcal{F}_{K} \times \mathcal{F}_{\mathbb{R}^{n}}\right)
$$

is a well defined morphism. Since this is a local question and we have $R^{-1}\left(\operatorname{Im} \bar{\varphi}^{-1}\right)=\operatorname{Im} \varphi^{-1} \times \operatorname{Id}_{\mathbb{R}^{d+1}}$, it suffices to prove that the induced map

$$
P^{*}: \Omega_{\bar{p}}^{*}\left(U \times \mathbb{R}^{n} / \mathcal{F}_{K / H} \times \mathcal{F}_{\mathbb{R}^{n}}\right) \rightarrow \Omega_{\bar{p}}^{*}\left(U \times H \times \mathbb{R}^{n} / \mathcal{F}_{K / H} \times \mathcal{F}_{H} \times \mathcal{F}_{\mathbb{R}^{n}}\right)
$$

is well defined. This follows from the fact that the map

$\nabla:\left(U \times H \times \mathbb{R}^{n}, \mathcal{F}_{K / H} \times \mathcal{F}_{H} \times \mathcal{F}_{\mathbb{R}^{n}}\right) \rightarrow\left(U \times H \times \mathbb{R}^{n}, \mathcal{F}_{K / H} \times \mathcal{F}_{H} \times \mathcal{F}_{\mathbb{R}^{n}}\right)$

defined by $\nabla(u, h, z)=(u, h, \Theta(h, z))$ is a foliated diffeomorphism. Since $\mathrm{pr}_{0} \circ \nabla=R$ with $\mathrm{pr}_{0}: U \times H \times \mathbb{R}^{n} \rightarrow U \times \mathbb{R}^{n}$ being the canonical projection, it suffices to apply 3.4.1(f) and Proposition 3.5.1. The injectivity of $R^{*}$ follows from the fact that $R$ is a surjection.

3.8.2. Lemma. Let $\Phi: G \times M \rightarrow M$ be a tame action. Let $K$ be a connected tamer group of $G$. Write $V_{u} \in \mathcal{X}(K)$ for the fundamental vector field associated to an element $u$ of the Lie algebra of $K$. For any perversity $\bar{p}$ the contraction operator

$$
i_{V_{u}}:\left(\Omega_{\bar{p}}^{*}(M / \mathcal{F})\right)^{K} \rightarrow\left(\Omega_{\bar{p}}^{*-1}(M / \mathcal{F})\right)^{K}
$$

is well defined.

Proof. Since $K$ is connected, the vector field $V_{u}$ is $K$-invariant. So, the contraction operator $i_{V_{u}}$ preserves the $K$-invariant differential forms. Moreover, if $\omega \in\left(\Omega_{\bar{p}}^{*}(M / \mathcal{F})\right)^{K}$ and $X$ is a vector field on $M$ tangent to the foliation $\mathcal{F}$ then

$$
i_{X} i_{V_{u}} \omega=-i_{V_{u}} i_{X} \omega=0 \quad \text { and } \quad i_{X} d i_{V_{u}} \omega=-i_{X} i_{V_{u}} d \omega=i_{V_{u}} i_{X} d \omega=0 .
$$

We end the proof if we show that $\omega \in\left(\Omega_{\bar{p}}^{*}\left(M \times\left[0,1\left[{ }^{p}\right)\right)^{K}\right.\right.$ implies $i_{V_{u}} \omega \in$ $\Omega_{\bar{p}}^{*-1}\left(M \times\left[0,1{ }^{p}\right)\right.$. We proceed by induction on depth $\mathrm{S}_{\mathcal{F}}$.

FIRST STEP: depth $S_{\mathcal{F}}=0$. The result is clear.

INDUCTION STEP. Since the question is local, we can assume that $M=T$ is a $K$-invariant tubular neighborhood of a singular stratum $S$. Let $\widetilde{V}_{u} \in$ $\mathcal{X}(D \times[0,1[)$ be the fundamental vector field associated to $u$. This field is tangent to the boundary of $D \times[0,1[$; write $U$ for its restriction. On the 
other hand, since $\nabla: D \times[0,1[\rightarrow T$ is a $K$-equivariant map, we obtain $\nabla_{*} \widetilde{V}_{u}=V_{u}$.

For each $\alpha \in \Omega^{*}(D)$ we have the implication $i_{U} \alpha\left(v_{1}, \ldots, v_{j}\right) \neq 0 \Rightarrow$ $\alpha\left(v_{1}, \ldots, v_{j}\right) \neq 0$. This gives the inequality $\left\|i_{U} \alpha\right\|_{\tau} \leq\|\alpha\|_{\tau}$.

Set $I=\operatorname{Id}_{[0,1}\left[{ }^{p}\right.$. We have $(\nabla \times I)^{*} \omega \in \Omega_{\bar{p}}^{*}\left(D \times\left[0,1\left[^{p+1}\right)\right.\right.$ with

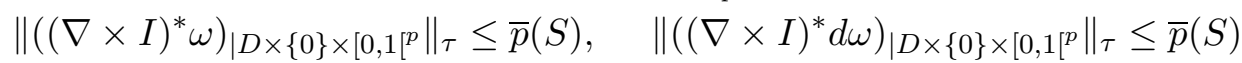
(cf. 3.4.1(d)). By induction hypothesis we have

$$
(\nabla \times I)^{*}\left(i_{V_{u}} \omega\right)=i_{\widetilde{V}_{u}}(\nabla \times I)^{*} \omega \in \Omega_{\bar{p}}^{*}\left(D \times\left[0,1\left[{ }^{p+1}\right) .\right.\right.
$$

The result now follows from

$$
\begin{aligned}
\|\left((\nabla \times I)^{*}\left(i_{V_{u}} \omega\right)\right)_{\mid D \times\{0\} \times\left[0,1\left[^{p} \|_{\tau}\right.\right.} & =\|\left(i_{\widetilde{V}_{u}}(\nabla \times I)^{*} \omega\right)_{\mid D \times\{0\} \times\left[0,1\left[^{p} \|_{\tau}\right.\right.} \\
& =\| i_{U}\left((\nabla \times I)^{*} \omega\right)_{\mid D \times\{0\} \times\left[0,1\left[^{p}\right) \|_{\tau}\right.} \\
& \leq \|\left((\nabla \times I)^{*} \omega\right)_{\mid D \times\{0\} \times\left[0,1{ }^{p} \|_{\tau} \leq \bar{p}(S),\right.}
\end{aligned}
$$

and

$$
\begin{aligned}
\|\left((\nabla \times I)^{*}\left(d\left(i_{V_{u}} \omega\right)\right)\right)_{\mid D \times\{0\} \times\left[0,1\left[^{p} \|_{\tau}\right.\right.} & =\|-\left((\nabla \times I)^{*}\left(i_{V_{u}} d \omega\right)\right)_{\mid D \times\{0\} \times\left[0,1\left[^{p} \|_{\tau}\right.\right.} \\
& \leq \bar{p}(S)
\end{aligned}
$$

since $\omega$ is $K$-invariant and 3.4.1(d) holds.

3.8.3. Fixing some notations for Lie algebras. Let $\mathfrak{k}, \mathfrak{g}$ and $\mathfrak{h}$ be the Lie algebras of $K, G$ and $H$ respectively. Choose an invariant riemannian metric $\kappa$ on $K$, which exists by compactness. Let

$$
\left\{u_{1}, \ldots, u_{a}, u_{a+1}, \ldots, u_{b}, u_{b+1}, \ldots, u_{c}, u_{c+1}, \ldots, u_{f}\right\}
$$

be an orthonormal basis of $\mathfrak{k}$ with $\left\{u_{1}, \ldots, u_{b}\right\}$ a basis of $\mathfrak{g}$ and $\left\{u_{a+1}, \ldots, u_{c}\right\}$ a basis of $\mathfrak{h}$.

For each index $i$ we let $X_{i} \in \mathcal{X}(K)$ be the invariant vector field associated to $u_{i}$ (cf. 2.1.1(a)). Let $\gamma_{i} \in \Omega^{1}(K)$ be the dual form of $X_{i}$, that is, $\gamma_{i}=i_{X_{i}} \kappa$. It is a cycle and it is $K$-invariant, that is, $k^{*} \gamma_{i}=\gamma_{i}$ for each $k \in K$. Since $K / H$ is an abelian Lie group, $H^{*}(K / H)=\Lambda^{*}\left(\gamma_{1}, \ldots, \gamma_{a}, \gamma_{c+1}, \ldots, \gamma_{f}\right)$. The $\mathcal{F}_{K / H^{-}}$basic differential forms on $\Lambda\left(\gamma_{1}, \ldots, \gamma_{a}, \gamma_{c+1}, \ldots, \gamma_{f}\right)$ are exactly $\bigwedge^{*}\left(\gamma_{c+1}, \ldots, \gamma_{f}\right)$. This gives

$$
H^{*}\left((K / H) / \mathcal{F}_{K / H}\right)=\Lambda^{*}\left(\gamma_{c+1}, \ldots, \gamma_{f}\right)
$$

3.8.4. Proposition. Let $K \times_{H} \mathbb{R}^{n}$ be a twisted product. Suppose that the group $G$ is connected and dense in $K$. Then

$$
\mathbb{H}_{\bar{p}}^{*}\left(K \times_{H} \mathbb{R}^{n} / \mathcal{F}_{\mathrm{tw}}\right) \cong H^{*}\left((K / H) / \mathcal{F}_{K / H}\right) \otimes\left(\mathbb{H}_{\bar{p}}^{*}\left(\mathbb{R}^{n} / \mathcal{F}_{\mathbb{R}^{n}}\right)\right)^{H}
$$

for any perversity $\bar{p}$.

Proof. Since $R^{*}: \Omega_{\bar{p}}^{*}\left(K \times H \mathbb{R}^{n} / \mathcal{F}_{\text {tw }}\right) \rightarrow \Omega_{\bar{p}}^{*}\left(K \times \mathbb{R}^{n} / \mathcal{F}_{K} \times \mathcal{F}_{\mathbb{R}^{n}}\right)$ is a monomorphism (cf. Lemma 3.8.1), it suffices to compute the cohomology of $\operatorname{Im} R^{*}$. We describe this complex in several steps. 
〈i Description of $\Omega^{*}\left(K \times R_{\mathcal{F}_{\mathbb{R}^{n}}}\right)$. A differential form in $\Omega^{*}\left(K \times R_{\mathcal{F}_{\mathbb{R}^{n}}}\right)$ is of the form

$$
\eta+\sum_{1 \leq i_{1}<\cdots<i_{\ell} \leq f} \gamma_{i_{1}} \wedge \cdots \wedge \gamma_{i_{\ell}} \wedge \eta_{i_{1}, \ldots, i_{\ell}}
$$

where $\eta, \eta_{i_{1}, \ldots, i_{\ell}} \in \Omega^{*}\left(K \times R_{\mathcal{F}^{n}}\right)$ satisfy $i_{X_{i}} \eta=i_{X_{i}} \eta_{i_{1}, \ldots, i_{\ell}}=0$ for all indices.

〈ii Description of $\Pi_{\mathcal{F}_{K} \times \mathcal{F}_{\mathbb{R}^{n}}}\left(K \times \mathbb{R}^{n}\right)$. Since the foliation $\mathcal{F}_{K}$ is regular, we can always choose a conical chart of the form $\left(U_{1} \times U_{2}, \varphi_{1} \times \varphi_{2}\right)$ where $\left(U_{1}, \varphi_{1}\right)$ is a foliated chart of $\left(K, \mathcal{F}_{K}\right)$ and $\left(U_{2}, \varphi_{2}\right)$ is a conical chart of $\left(\mathbb{R}^{n}, \mathcal{F}_{\mathbb{R}^{n}}\right)$. The local blow-up of the chart $\left(U_{1} \times U_{2}, \varphi_{1} \times \varphi_{2}\right)$ is constructed from the second factor without modifying the first one. So, the differential forms $\gamma_{i}$ are always perverse and any $\omega \in \Pi_{\mathcal{F}_{K} \times \mathcal{F}_{\mathbb{R}^{n}}}^{*}\left(K \times \mathbb{R}^{n}\right)$ is of the form (16) where $\eta, \eta_{i_{1}, \ldots, i_{\ell}} \in \Pi_{\mathcal{F}_{K} \times \mathcal{F}_{\mathbb{R}^{n}}}^{*}\left(K \times \mathbb{R}^{n}\right)$ satisfy $i_{X_{i}} \eta=i_{X_{i}} \eta_{i_{1}, \ldots, i_{\ell}}=0$ for all indices.

〈iii Description of $\Omega^{*}\left(K \times R_{\mathcal{F}_{\mathbb{R}^{n}}} / \mathcal{F}_{K} \times \mathcal{F}_{\mathbb{R}^{n}}\right)$. Let $\omega \in \Omega^{*}\left(K \times R_{\mathcal{F}_{\mathbb{R}^{n}}} /\right.$ $\left.\mathcal{F}_{K} \times \mathcal{F}_{\mathbb{R}^{n}}\right)$. Denote by

$$
\aleph: K \times\left(K \times \mathbb{R}^{n}\right) \rightarrow K \times \mathbb{R}^{n}
$$

the action defined by $\aleph(g,(k, u))=(g \cdot k, u)$ and by $\aleph_{G}: G \times\left(K \times \mathbb{R}^{n}\right) \rightarrow$ $K \times \mathbb{R}^{n}$ its restriction. Since the fundamental vector fields of the action $\aleph_{G}$ are tangent to the foliation $\mathcal{F}_{K} \times \mathcal{F}_{\mathbb{R}^{n}}, \omega$ is $G$-invariant and, by density, also $K$-invariant. So, $\omega \in \bigwedge^{*}\left(\gamma_{1}, \ldots, \gamma_{f}\right) \otimes \Omega^{*}\left(R_{\mathcal{F}_{\mathbb{R}^{n}}}\right)$. We need here the connectedness of $G$.

Since the $\mathcal{F}_{K}$-basic differential forms in $\Lambda^{*}\left(\gamma_{1}, \ldots, \gamma_{f}\right)$ are exactly $\Lambda^{*}\left(\gamma_{b+1}, \ldots, \gamma_{f}\right)$, we get a differential isomorphism

$$
\Omega^{*}\left(K \times R_{\mathcal{F}_{\mathbb{R}^{n}}} / \mathcal{F}_{K} \times \mathcal{F}_{\mathbb{R}^{n}}\right) \cong \Lambda^{*}\left(\gamma_{b+1}, \ldots, \gamma_{f}\right) \otimes \Omega^{*}\left(R_{\mathcal{F}_{\mathbb{R}^{n}}} / \mathcal{F}_{\mathbb{R}^{n}}\right) .
$$

$\langle$ iv $\rangle$ Description of $\Omega_{\bar{p}}^{*}\left(K \times \mathbb{R}^{n} / \mathcal{F}_{K} \times \mathcal{F}_{\mathbb{R}^{n}}\right)$. From $\langle$ ii $\rangle$ and $\langle$ iii $\rangle$ it suffices to control the perverse degree of the forms

$$
\eta+\sum_{b+1 \leq i_{1}<\cdots<i_{\ell} \leq f} \gamma_{i_{1}} \wedge \cdots \wedge \gamma_{i_{\ell}} \wedge \eta_{i_{1}, \ldots, i_{\ell}} \in \Lambda^{*}\left(\gamma_{b+1}, \ldots, \gamma_{f}\right) \otimes \Pi_{\mathcal{F}_{\mathbb{R}^{n}}^{*}}^{*}\left(\mathbb{R}^{n}\right) .
$$

Let $S$ be a stratum of $\mathrm{S}_{\mathcal{F}_{\mathbb{R}^{n}}}$. From $\left\|\gamma_{i}\right\|_{K \times S}=0$ and $\|\eta\|_{K \times S}=\|\eta\|_{S}$, we get $\left\|\gamma_{i_{1}} \wedge \cdots \wedge \gamma_{i_{\ell}} \wedge \eta_{i_{1}, \ldots, i_{\ell}}\right\|_{K \times S}=\left\|\eta_{i_{1}, \ldots, i_{\ell}}\right\|_{S}$. We conclude that

$$
\Omega_{\bar{p}}^{*}\left(K \times \mathbb{R}^{n} / \mathcal{F}_{K} \times \mathcal{F}_{\mathbb{R}^{n}}\right) \cong \bigwedge^{*}\left(\gamma_{b+1}, \ldots, \gamma_{f}\right) \otimes \Omega_{\bar{p}}^{*}\left(\mathbb{R}^{n} / \mathcal{F}_{\mathbb{R}^{n}}\right)
$$

as differential complexes.

$\langle\mathrm{v}\rangle$ Description of $\operatorname{Im}\left\{R^{*}: \Omega_{\bar{p}}^{*}\left(K \times_{H} \mathbb{R}^{n} / \mathcal{F}_{\text {tw }}\right) \rightarrow \Omega_{\bar{p}}^{*}\left(K \times \mathbb{R}^{n} / \mathcal{F}_{K} \times \mathcal{F}_{\mathbb{R}^{n}}\right)\right\}$. We denote by $\left\{W_{a+1}, \ldots, W_{c}\right\}$ the fundamental vector fields of the action $\Theta: H \times \mathbb{R}^{n} \rightarrow \mathbb{R}^{n}$. Consider now the action $\Upsilon: H \times\left(K \times \mathbb{R}^{n}\right) \rightarrow K \times \mathbb{R}^{n}$ defined by $\Upsilon(h,(k, z))=\left(k \cdot h^{-1}, \Theta(h, z)\right)$. The fundamental vector fields are 
$\left\{\left(-X_{a+1}, W_{a+1}\right), \ldots,\left(-X_{c}, W_{c}\right)\right\}$. Given $h \in H$ we let $\Upsilon_{h}: K \times \mathbb{R}^{n} \rightarrow K \times \mathbb{R}^{n}$ be defined by $\Upsilon_{h}(k, z)=\Upsilon(h,(k, z))$. Then

$\operatorname{Im} R^{*}=\left\{\omega \in \Lambda^{*}\left(\gamma_{b+1}, \ldots, \gamma_{f}\right) \otimes \Omega_{\bar{p}}^{*}\left(\mathbb{R}^{n} / \mathcal{F}_{\mathbb{R}^{n}}\right) \mid\right.$ (i) $i_{X_{i}} \omega=i_{W_{i}} \omega$ if $a+1 \leq i \leq c$

(ii) $\left(\Upsilon_{h}\right)^{*} \omega=\omega$ for $\left.h \in H\right\}$

(as differential complexes). Recall that $h^{*} \gamma_{i}=\gamma_{i}$ for all $h \in H$ and $i$. The foliation $\mathcal{F}_{\mathbb{R}^{n}}$ is defined by the action of $G \cap H$ and so $i_{W_{i}} \omega=0$ for $i \in\{a+1, \ldots, b\}$. Since $\gamma_{i}\left(X_{j}\right)=\delta_{i j}$, we deduce that $\operatorname{Im} R^{*}$ is the differential complex

$\left\{\omega \in \bigwedge^{*}\left(\gamma_{b+1}, \ldots, \gamma_{f}\right) \otimes\left(\Omega_{\bar{p}}^{*}\left(\mathbb{R}^{n} / \mathcal{F}_{\mathbb{R}^{n}}\right)\right)^{H} \mid i_{X_{i}} \omega=i_{W_{i}} \omega\right.$ if $\left.b+1 \leq i \leq c\right\}$

$=\bigwedge^{*}\left(\gamma_{c+1}, \ldots, \gamma_{f}\right)$

$\otimes \underbrace{\left\{\omega \in \Lambda^{*}\left(\gamma_{b+1}, \ldots, \gamma_{c}\right) \otimes\left(\Omega_{\bar{p}}^{*}\left(\mathbb{R}^{n} / \mathcal{F}_{\mathbb{R}^{n}}\right)\right)^{H} \mid i_{X_{i}} \omega=i_{W_{i}} \omega \text { if } b+1 \leq i \leq c\right\}}_{A^{*}}$.

Define $\Delta:\left(\Omega_{\bar{p}}^{*}\left(\mathbb{R}^{n} / \mathcal{F}_{\mathbb{R}^{n}}\right)\right)^{H} \rightarrow \bigwedge^{*}\left(\gamma_{b+1}, \ldots, \gamma_{c}\right) \otimes\left(\Omega_{\bar{p}}^{*}\left(\mathbb{R}^{n} / \mathcal{F}_{\mathbb{R}^{n}}\right)\right)^{H}$ by

$$
\Delta(\beta)=\beta+\sum_{b+1 \leq i_{1}<\cdots<i_{\ell} \leq c} \gamma_{i_{1}} \wedge \cdots \wedge \gamma_{i_{\ell}} \wedge\left(i_{W_{i_{\ell}}} \cdots i_{W_{i_{1}}} \beta\right) .
$$

A straightforward computation shows that $\Delta$ is a differential operator and its restriction $\Delta:\left(\Omega_{\bar{p}}^{*}\left(\mathbb{R}^{n} / \mathcal{F}_{\mathbb{R}^{n}}\right)\right)^{H} \rightarrow A^{*}$ is an isomorphism. The inverse operator is

$$
\Delta^{-1}\left(\xi_{0}+\sum_{b+1 \leq i_{1}<\cdots<i_{\ell} \leq c} \gamma_{i_{1}} \wedge \cdots \wedge \gamma_{i_{\ell}} \wedge \xi_{i_{1}, \ldots, i_{\ell}}\right)=\xi_{0}
$$

(cf. Lemma 3.8.2). We conclude that the differential complex $\operatorname{Im} R^{*}$ is isomorphic to

$$
\bigwedge^{*}\left(\gamma_{c+1}, \ldots, \gamma_{f}\right) \otimes\left(\Omega_{\bar{p}}^{*}\left(\mathbb{R}^{n} / \mathcal{F}_{\mathbb{R}^{n}}\right)\right)^{H}
$$

$\langle$ vi Last step. Consider now the operator

$$
H^{*}\left(\left(\Omega_{\bar{p}}^{*}\left(\mathbb{R}^{n} / \mathcal{F}_{\mathbb{R}^{n}}\right)\right)^{H}\right) \hookrightarrow\left(\mathbb{H}_{\bar{p}}^{*}\left(\mathbb{R}^{n} / \mathcal{F}_{\mathbb{R}^{n}}\right)\right)^{H}
$$

induced by the inclusion $\iota:\left(\Omega_{\bar{p}}^{*}\left(\mathbb{R}^{n} / \mathcal{F}_{\mathbb{R}^{n}}\right)\right)^{H} \hookrightarrow \Omega_{\bar{p}}^{*}\left(\mathbb{R}^{n} / \mathcal{F}_{\mathbb{R}^{n}}\right)$. The usual arguments show that this operator is an isomorphism:

- Monomorphism: $\omega=d \eta \Rightarrow \omega=d \int_{H} \Upsilon_{h}^{*} \eta d h$.

- Epimorphism: $\omega-\Upsilon_{h}^{*} \omega=d \eta_{h}$ for each $h \in H \Rightarrow \omega-\int_{H} \Upsilon_{h}^{*} \omega d h=$ $d \int_{H} \eta_{h} d h$.

We get

$$
I_{\bar{p}}^{*}\left(K \times_{H} \mathbb{R}^{n} / \mathcal{F}_{\mathrm{tw}}\right) \cong H^{*}\left((K / H) / \mathcal{F}_{K / H}\right) \otimes\left(\mathbb{H}_{\bar{p}}^{*}\left(\mathbb{R}^{n} / \mathcal{F}_{\mathbb{R}^{n}}\right)\right)^{H}
$$


3.8.5. REMARK. The same procedure shows that the differential operator

$$
\nabla=\left(\operatorname{Id}_{\bigwedge^{*}\left(\gamma_{1}, \ldots, \gamma_{a}, \gamma_{c+1}, \ldots, \gamma_{f}\right)} \otimes \Delta^{-1}\right) \circ R^{*}
$$

gives an isomorphism

$$
\begin{aligned}
\left(\Pi_{\mathcal{F}_{\text {tw }}}^{*}\left(K \times_{H} \mathbb{R}^{n}\right)\right)^{K} & \cong \bigwedge^{*}\left(\gamma_{1}, \ldots, \gamma_{a}, \gamma_{c+1}, \ldots, \gamma_{f}\right) \otimes\left(\Pi_{\mathcal{F}_{\mathbb{R}^{n}}}^{*}\left(\mathbb{R}^{n}\right)\right)^{H} \\
& =H^{*}(K / H) \otimes\left(\Pi_{\mathcal{F}_{\mathbb{R}^{n}}}^{*}\left(\mathbb{R}^{n}\right)\right)^{H}
\end{aligned}
$$

\section{COHOMOLOGICAL PROPERTIES OF THE BIC}

In this section we prove that the BIC of a compact foliated manifold $(M, \mathcal{F})$, determined by an abelian isometric action $\Phi: G \times M \rightarrow M$, is finite-dimensional and satisfies the Poincaré duality.

If the orbits of this action have the same dimension, that is, depth $\mathrm{S}_{\mathcal{F}}=0$, then $\mathcal{F}$ is a (regular) riemannian foliation (cf. [12]) and the BIC becomes the usual basic cohomology $H^{*}(M / \mathcal{F})$. We already know from [7] that $H^{*}(M / \mathcal{F})$ is finite-dimensional and satisfies the Poincaré duality. For the general case we are going to proceed by induction on depth $S_{\mathcal{F}}$.

But first of all we show how the BIC generalizes the usual basic cohomology. The same situation appears for the intersection homology of a stratified pseudomanifold (cf. [8]).

4.1. Proposition. Let $(M, \mathcal{F})$ be a foliated manifold determined by a tame action. Then

(i) $I H_{\bar{q}}^{*}(M / \mathcal{F}) \cong H^{*}\left(R_{\mathcal{F}} / \mathcal{F}\right)$ if $\bar{q}>\bar{t}$.

(ii) $H_{\overline{0}}^{*}(M / \mathcal{F}) \cong H^{*}(M / \mathcal{F})$.

(iii) $\mathbb{H}_{\bar{p}}^{*}(M / \mathcal{F}) \cong H^{*}\left(\left(M, \Sigma_{\mathcal{F}}\right) / \mathcal{F}\right)$ if $\bar{p}<0$.

Proof. The map $\omega \mapsto \omega$ gives a differential operator $I_{M}: \Omega_{\bar{q}}^{*}(M / \mathcal{F}) \rightarrow$ $\Omega^{*}\left(R_{\mathcal{F}} / \mathcal{F}\right)$. The restriction map $\omega \mapsto \omega_{R_{\mathcal{F}}}$ defines differential operators $J_{M}: \Omega^{*}(M / \mathcal{F}) \rightarrow \Omega_{\overline{0}}^{*}(M / \mathcal{F})$ and $K_{M}: \Omega^{*}\left(\left(M, \Sigma_{\mathcal{F}}\right) / \mathcal{F}\right) \rightarrow \Omega_{\bar{p}}^{*}(M / \mathcal{F})$ (cf. 3.2.1(b)). We prove by induction on depth $\mathrm{S}_{\mathcal{F}}$ the following assertions:

$$
\begin{aligned}
& \mathfrak{A}_{1}(M, \mathcal{F})=\text { " } I_{M} \text { is a quasi-isomorphism", } \\
& \mathfrak{A}_{2}(M, \mathcal{F})=\text { " } J_{M} \text { is a quasi-isomorphism", } \\
& \mathfrak{A}_{3}(M, \mathcal{F})=\text { " } K_{M} \text { is a quasi-isomorphism". }
\end{aligned}
$$

FIRST STEP: depth $S_{\mathcal{F}}=0$. The singular part $\Sigma_{\mathcal{F}}$ is empty and therefore $I_{\bar{q}}^{*}(M / \mathcal{F})=I H_{\overline{0}}^{*}(M / \mathcal{F})=I H_{\bar{p}}^{*}(M / \mathcal{F})=H^{*}(M / \mathcal{F})$ with $I_{M}=J_{M}=$ $K_{M}=\mathrm{Id}$.

InduCtion step. The family $\left\{M-S_{\min }, T_{\min }\right\}$ is a $K$-invariant open covering of $M$ (cf. 2.4). Choose a smooth map $\alpha:[0,1[\rightarrow \mathbb{R}$ with $\alpha \equiv 1$ on $\left[0,1 / 4\left[\right.\right.$ and $\alpha \equiv 0$ on $\left[3 / 4,1\left[\right.\right.$. Write $f=\alpha \circ \varrho_{\min }: M \rightarrow \mathbb{R}$, which is a $K$ invariant map and therefore $\mathcal{F}$-basic. Since supp $f \subset T_{\min }$ and $\operatorname{supp}(1-f) \subset$ 
$M-S_{\min }$, the covering is basic. From 3.6 we have a Mayer-Vietoris sequence and we get

$$
\mathfrak{A}_{i}\left(T_{\text {min }}-S_{\text {min }}, \mathcal{F}\right) \& \mathfrak{A}_{i}\left(M-S_{\text {min }}, \mathcal{F}\right) \& \mathfrak{A}_{i}\left(T_{\text {min }}, \mathcal{F}\right) \Rightarrow \mathfrak{A}_{i}(M, \mathcal{F}),
$$

for $i=1,2,3$. The induction hypothesis yields $\mathfrak{A}_{i}\left(T_{\min }-S_{\min }, \mathcal{F}\right)$ and $\mathfrak{A}_{i}(M-$ $\left.S_{\text {min }}, \mathcal{F}\right)$; it remains to prove $\mathfrak{A}_{i}\left(T_{\min }, \mathcal{F}\right)$.

From 3.2.1(d) we know that we can identify the perverse forms on $T_{\min }$ with those on $D_{\min } \times[0,1$. This identification sends basic forms to basic forms and preserves the perverse degrees relative to any stratum different from those of $S_{\min }$. The perverse degree of a perverse form $\omega$ on $D_{\min } \times[0,1$ [ relative to $S_{\min }$ becomes the vertical degree of the restriction of $\omega$ relative to $\nabla_{\text {min }} \equiv \tau_{\text {min }}: D_{\text {min }} \times\{0\} \equiv D_{\text {min }} \rightarrow S_{\text {min }}($ cf. $3.2 .1(\mathrm{~d}))$. That is,

$$
\begin{aligned}
& \Omega_{\bar{q}}^{*}\left(T_{\min } / \mathcal{F}\right) \quad \text { becomes } \Omega_{\bar{q}}^{*}\left(D_{\min } \times[0,1[/ \mathcal{F} \times \mathcal{I}) \quad(\text { cf. 3.4.1(b)), }\right. \\
& \Omega_{\overline{0}}^{*}\left(T_{\min } / \mathcal{F}\right) \text { becomes }\left\{\omega \in \Omega _ { \overline { p } } ^ { * } \left(D_{\min } \times[0,1[/ \mathcal{F} \times \mathcal{I}) \mid\right.\right. \\
& \left.\omega_{\mid D_{\min }}=\tau_{\min }^{*} \eta \text { with } \eta \in \Omega^{*}\left(S_{\min } / \mathcal{F}\right)\right\}, \\
& \Omega_{\bar{p}}^{*}\left(T_{\min } / \mathcal{F}\right) \text { becomes }\left\{\omega \in \Omega _ { \overline { p } } ^ { * } \left(D_{\min } \times\left[0,1[/ \mathcal{F} \times \mathcal{I}) \mid \omega_{\mid D_{\min }}=0\right\}\right.\right. \text {. }
\end{aligned}
$$

Proceeding as in Proposition 3.5.1 we prove that

$$
\begin{gathered}
\mathbb{H}_{\bar{q}}^{*}\left(T_{\min } / \mathcal{F}\right) \cong H_{\bar{q}}^{*}\left(D_{\min } / \mathcal{F}\right), \quad \mathbb{H}_{\overline{0}}^{*}\left(T_{\min } / \mathcal{F}\right) \cong H^{*}\left(S_{\min } / \mathcal{F}\right), \\
\mathbb{H}_{\bar{p}}^{*}\left(T_{\min } / \mathcal{F}\right) \cong 0 .
\end{gathered}
$$

Notice that $I_{T_{\min }}$ is induced by pro $\left.\nabla_{\min }^{-1}: T_{\min }-\Sigma_{\mathcal{F}} \rightarrow D_{\min } \times\right] 0,1\left[\rightarrow D_{\min }\right.$ and $J_{T}$ becomes $\tau^{*}$.

On the other hand, since $\nabla_{\min }:\left(D_{\min } \times\right] 0,1[, \mathcal{F} \times \mathcal{I}) \rightarrow\left(T_{\min }-\Sigma_{\mathcal{F}}, \mathcal{F}\right)$ is a foliated diffeomorphism, we get

$$
H^{*}\left(\left(T_{\min }-\Sigma_{\mathcal{F}}\right) / \mathcal{F}\right) \cong H^{*}\left(D_{\min } \times\right] 0,1[/ \mathcal{F} \times \mathcal{I}) \stackrel{3.5 .1}{\cong} H^{*}\left(D_{\min } / \mathcal{F}\right),
$$

where the isomorphism is induced by pr $\circ \nabla^{-1}$. The induction hypothesis implies that $I_{T_{\min }}$ is a quasi-isomorphism.

The inclusion $\iota_{\min }: S_{\min } \rightarrow T_{\min }$ and the projection $\tau_{\min }: T_{\min } \rightarrow S_{\min }$ are foliated maps with $\tau_{\min } \circ \iota_{\text {min }}=\mathrm{Id}$, so they induce operators $\iota^{*}: \Omega^{*}\left(S_{\min } / \mathcal{F}\right)$ $\rightarrow \Omega^{*}\left(T_{\min } / \mathcal{F}\right)$ and $\tau^{*}: \Omega^{*}\left(T_{\min } / \mathcal{F}\right) \rightarrow \Omega^{*}\left(S_{\min } / \mathcal{F}\right)$ satisfying $\iota_{\text {min }}^{*} \circ \tau_{\min }^{*}=$ Id. The composition $\iota_{\min } \circ \tau_{\min }$ is homotopic to the identity by a foliated homotopy. This homotopy is just $H: T_{\min } \times[0,1] \rightarrow T_{\min }$ (cf. 2.4). So, $\tau_{\min }^{*}$ induces an isomorphism

$$
H^{*}\left(T_{\min } / \mathcal{F}\right) \cong H^{*}\left(S_{\min } / \mathcal{F}\right) .
$$

This proves that $J_{T_{\min }}$ is a quasi-isomorphism.

Let $\omega \in \Omega^{*}\left(\left(T_{\min }, \Sigma_{\mathcal{F}}\right) / \mathcal{F}\right)$ be a cycle. The above homotopy operator gives the relation $\omega=d \int_{0}^{1} H^{*} \omega$. Since the homotopy $H$ preserves $\mathcal{F}$, we have $\int_{0}^{1} H^{*} \omega \in \Omega^{*}\left(\left(T, \Sigma_{\mathcal{F}}\right) / \mathcal{F}\right)$ and therefore $H^{*}\left(\left(T_{\min }, \Sigma_{\mathcal{F}}\right) / \mathcal{F}\right)=0$. This proves that $K_{T_{\min }}$ is a quasi-isomorphism. 
4.2. Finiteness. We prove that the BIC of the conical foliation $\mathcal{F}$ induced by an abelian isometric action on a compact manifold is finite-dimensional. We proceed by induction on depth $S_{\mathcal{F}}$. In order to decrease the depth we use the blow-up of Molino. This will lead us to the twisted product through the invariant tubular neighborhood.

4.2.1. Proposition. Let $(M, \mathcal{F})$ be a conical foliated manifold determined by a tame action. Let $\left(T, \tau, S, \mathbb{R}^{n}\right)$ be a $K$-invariant tubular neighborhood of a compact singular stratum $S$. If the BIC of the slice $\left(\mathbb{R}^{n}, \mathcal{F}_{\mathbb{R}^{n}}\right)$ is finite-dimensional then so is the BIC of the tube $(T, \mathcal{F})$.

Proof. We can suppose that $G$ is connected and we fix a connected tamer group $K$ in which $G$ is dense. We consider the orbit type stratification induced by the action $\Phi: K \times S \rightarrow S$ (cf. 2.5). We prove by induction on depth $S_{\Phi}$ the following statement.

$\mathfrak{A}(T, \mathcal{F})=$ "The BIC $\mathbb{H}_{\bar{p}}^{*}(T / \mathcal{F})$ is finite-dimensional for each perversity $\bar{p}$.

Fix $\bar{p}$. Recall that any $K$-invariant submanifold of $M$ inherits naturally a perversity (cf. 3.4), still denoted $\bar{p}$. We proceed in two steps.

First STEP: depth $\mathrm{S}_{\Phi}=0$. The isotropy subgroup of any point of $S$ is a compact subgroup $H \subset K$. The orbit space $S / K$ is a manifold and the natural projection $\pi: S \rightarrow S / K$ is a locally trivial bundle with fiber $K / H$. Fix a good open covering $\left\{U_{\alpha}\right\}$ of $S / K$ (cf. [4]), closed under finite intersections. For an open subset $U \subset S / K$ we consider the statement

$$
Q(U)=\text { "The BIC } \mathbb{H}_{\bar{p}}^{*}\left(\tau^{-1} \pi^{-1}(U) / \mathcal{F}\right) \text { is finite-dimensional". }
$$

Notice that $Q(S)=\mathfrak{A}(T, \mathcal{F})$. We get the result if we verify the three conditions of Bredon's trick (3.6.1).

(BT1) Since $U_{\alpha}$ is contractible we can identify $\pi^{-1}\left(U_{\alpha}\right)$ with $U_{\alpha} \times K / H$. The group $K$ acts by $k_{0} \cdot\left(x, k_{1} H\right)=\left(x, k_{0} k_{1} H\right)$. Fix a base point $x_{0}$ in $U_{\alpha}$ and identify $\left\{x_{0}\right\} \times K / H$ with $K / H$. The contractibility of $U_{\alpha}$ gives a $K$-invariant $O(n)$-isomorphism between $\left(T, \tau, S, \mathbb{R}^{n}\right)$ and $\left(U_{\alpha} \times \tau^{-1}(K / H), \operatorname{Id}_{U_{\alpha}} \times \tau, U_{\alpha} \times\right.$ $\left.K / H, \mathbb{R}^{n}\right)$. Notice that, identifying $\tau^{-1}(e H)$ with $\mathbb{R}^{n}$, the map $\langle k, u\rangle \mapsto k \cdot u$ realizes a $K$-diffeomorphism between $\tau^{-1}(K / H)$ and the twisted product $K \times{ }_{H} \mathbb{R}^{n}$.

The contractibility of $U_{\alpha}$ gives

$$
\begin{aligned}
\mathbb{H}_{\bar{p}}^{*}\left(\tau^{-1} \pi^{-1}\left(U_{\alpha}\right) / \mathcal{F}\right) & \cong H_{\bar{p}}^{*}\left(U_{\alpha} \times \tau^{-1}(K / H) / \mathcal{I} \times \mathcal{F}\right) \\
& \stackrel{3.5 .1}{\cong} H_{\bar{p}}^{*}\left(\tau^{-1}(K / H) / \mathcal{F}\right) \cong H_{\bar{p}}^{*}\left(K \times_{H} \mathbb{R}^{n} / \mathcal{F}\right),
\end{aligned}
$$

which is finite-dimensional by Proposition 3.8.4.

(BT2) The lifting of a partition of unity subordinate to the covering $\{U, V\}$ of $S / K$ gives a controlled and basic partition of unity subordinate 
to the covering $\left\{\tau^{-1} \pi^{-1}(U), \tau^{-1} \pi^{-1}(V)\right\}$ of $T$ (cf. 3.2.1(b)). This covering is basic. Now we apply 3.6.

(BT3) is clear since

$$
\mathbb{H}_{\bar{p}}^{*}\left(\left(\tau^{-1} \pi^{-1}\left(\bigcup_{i=0}^{m} U_{i}\right)\right) / \mathcal{F}\right)=\bigoplus_{i=0}^{m} \mathbb{H}_{\bar{p}}^{*}\left(\tau^{-1} \pi^{-1}\left(U_{i}\right) / \mathcal{F}\right) .
$$

Notice that the compactness required in the statement of the proposition is used here by considering a finite covering $\left\{U_{i} \mid 1 \leq i \leq m\right\}$. For an infinite covering, property (BT3) could be false.

InduCTION STEP. Denote by $S^{\text {min }}$ the union of the closed (minimal) strata of $\mathrm{S}_{\Phi}$ and choose a disjoint family $T^{\mathrm{min}}$ of $K$-invariant tubular neighborhoods of the closed strata. The projection map is denoted by $\tau^{\min }: T^{\min }$ $\rightarrow S^{\mathrm{min}}$. This family is not empty since depth $\mathrm{S}_{\Phi}>0$. The union of the associated tubes is denoted by $D^{\text {min }}$. It is a compact $K$-invariant submanifold satisfying

$$
\operatorname{depth} \mathrm{S}_{\Phi: K \times D^{\min } \rightarrow D^{\min }}<\operatorname{depth} \mathrm{S}_{\Phi} .
$$

The induced map $\left.\nabla^{\text {min }}: D^{\text {min }} \times\right] 0,1\left[\rightarrow T^{\text {min }}-S^{\text {min }}\right.$ is a $K$-equivariant diffeomorphism with trivial action on the $] 0,1\left[\right.$-factor. The radius map $\varrho^{\min }: T^{\mathrm{min}}$ $\rightarrow[0,1[$ is $K$-invariant.

The family $\left\{\tau^{-1}\left(S-S^{\text {min }}\right), \tau^{-1}\left(T^{\text {min }}\right)\right\}$ is a $K$-invariant open covering of $T$. Choose a smooth map $\alpha:[0,1[\rightarrow \mathbb{R}$ with $\alpha \equiv 1$ on $[0,1 / 4[$ and $\alpha \equiv 0$ on $\left[3 / 4,1\left[\right.\right.$. Write $f=\alpha \circ \varrho^{\min } \circ \tau: T \rightarrow \mathbb{R}$, which is $K$-invariant and therefore $\mathcal{F}$-basic. Since $\operatorname{supp} f \subset \tau^{-1}\left(T^{\mathrm{min}}\right)$ and $\operatorname{supp}(1-f) \subset \tau^{-1}\left(S-S^{\mathrm{min}}\right)$, the covering is basic. From 3.6 we get an exact Mayer-Vietoris sequence

$$
\begin{array}{r}
0 \rightarrow \Omega_{\bar{p}}^{*}\left(\tau^{-1}\left(T^{\text {min }}-S^{\text {min }}\right) / \mathcal{F}\right) \rightarrow \Omega_{\bar{p}}^{*}\left(\tau^{-1}\left(S-S^{\text {min }}\right) / \mathcal{F}\right) \oplus \Omega_{\bar{p}}^{*}\left(\tau^{-1}\left(T^{\text {min }}\right) / \mathcal{F}\right) \\
\rightarrow \Omega_{\bar{p}}^{*}(T / \mathcal{F}) \rightarrow 0 .
\end{array}
$$

The Five Lemma gives

$\mathfrak{A}\left(\tau^{-1}\left(T^{\mathrm{min}}-S^{\mathrm{min}}\right), \mathcal{F}\right) \& \mathfrak{A}\left(\tau^{-1}\left(T^{\mathrm{min}}\right), \mathcal{F}\right) \& \mathfrak{A}\left(\tau^{-1}\left(S-S^{\mathrm{min}}\right), \mathcal{F}\right) \Rightarrow \mathfrak{A}(T, \mathcal{F})$.

We now check the three premises.

(a) $\mathfrak{A}\left(\tau^{-1}\left(T^{\mathrm{min}}-S^{\mathrm{min}}\right) \mathcal{F}\right)$ : The $K$-equivariant diffeomorphism $\nabla^{\text {min }}$ produces by pull-back the commutative diagram

$$
\begin{aligned}
& \left.\tau^{-1}\left(D^{\mathrm{min}}\right) \times\right] 0,1\left[\stackrel{\widetilde{\nabla_{\min }}}{\longrightarrow} \tau^{-1}\left(T^{\mathrm{min}}-S^{\mathrm{min}}\right)\right. \\
& \tau \times \operatorname{Id}_{0,1} \downarrow \downarrow \tau \downarrow \\
& \left.D^{\min } \times\right] 0,1\left[\stackrel{\nabla^{\min }}{\longrightarrow} T^{\text {min }}-S^{\text {min }}\right.
\end{aligned}
$$


where $\widetilde{\nabla^{\text {min }}}$ is a $K$-diffeomorphism. So, we get

$$
\begin{aligned}
\mathbb{H}_{\bar{p}}^{*}\left(\tau^{-1}\left(T^{\mathrm{min}}-S^{\mathrm{min}}\right) / \mathcal{F}\right) & \cong \mathbb{H}_{\bar{p}}^{*}\left(\tau^{-1}\left(D^{\min }\right) \times\right] 0,1[/ \mathcal{F} \times \mathcal{I}) \\
& \stackrel{3.5 .1}{\cong} H_{\bar{p}}^{*}\left(\tau^{-1}\left(D^{\mathrm{min}}\right) / \mathcal{F}\right)
\end{aligned}
$$

which is finite from the induction hypothesis (see $(17)$ ).

(b) $\mathfrak{A}\left(\tau^{-1}\left(T^{\min }\right), \mathcal{F}\right)$ : The idea is the following. We prove that the inclusion $\tau^{-1}\left(S^{\text {min }}\right) \hookrightarrow \tau^{-1}\left(T^{\text {min }}\right)$ induces an isomorphism

$$
\left.\left.\mathbb{H}_{\bar{p}}^{*}\left(\tau^{-1}\left(T^{\min }\right) / \mathcal{F}\right)\right) \cong \mathbb{H}_{\bar{p}}^{*}\left(\tau^{-1}\left(S^{\min }\right) / \mathcal{F}\right)\right) .
$$

Now, since the depth of $\mathrm{S}_{\Phi: K \times S^{\min } \rightarrow S^{\min }}$ is 0 , it suffices to apply the first step.

The contraction $H^{\min }: T^{\min } \times[0,1] \rightarrow T^{\text {min }}$ is a $K$-invariant map with $H_{0}^{\text {min }}=\iota \circ \tau^{\text {min }}$ and $H_{1}^{\text {min }}=\operatorname{Id}_{\tau^{-1}\left(T^{\text {min }}\right)}$, where $\iota: S^{\text {min }} \hookrightarrow T^{\text {min }}$ is the natural inclusion. Notice that $\tau^{\min } \circ \iota=\operatorname{Id}_{\tau^{-1}\left(S^{\min }\right)}$. The map $H^{\text {min }}$ is locally the $\operatorname{map} H^{\text {min }}: U \times \mathbb{R}^{m} \times[0,1] \rightarrow U \times \mathbb{R}^{m}$ defined by $H^{\min }(x, v, t)=(x, t v)$.

Consider the commutative diagram induced by pull-back,

$$
\begin{array}{ccc}
\tau^{-1}\left(T^{\mathrm{min}}\right) \times[0,1] & \stackrel{\widehat{H^{\mathrm{min}}}}{\longrightarrow} \tau^{-1}\left(T^{\mathrm{min}}\right) \\
\tau \times \operatorname{Id}_{[0,1]} \downarrow & \tau \downarrow \\
T^{\mathrm{min}} \times[0,1] & \stackrel{H^{\mathrm{min}}}{\longrightarrow} & T^{\mathrm{min}}
\end{array}
$$

Let $\widetilde{\tau^{\min }}$ and $\widetilde{\iota}$ be the pull-backs of $\tau^{\text {min }}$ and $\iota$ respectively. We have $\left(\widetilde{H^{\text {min }}}\right)_{1}$ $=\tau \circ \widetilde{\tau^{\min }}$ and $\operatorname{Id}=\left(\widetilde{H^{\min }}\right)_{1}=\widetilde{\tau^{\text {min }}} \circ \widetilde{\iota}$.

The operator $\widetilde{H^{\text {min }}}$ is $K$-invariant and therefore is a foliated morphism: $\left(\widetilde{H^{\text {min }}}\right)^{*} \mathcal{F}=\mathcal{F} \times \mathcal{I}$. It is locally of the form $\widetilde{H^{\text {min }}}: U \times \mathbb{R}^{m} \times \mathbb{R}^{n} \times[0,1] \rightarrow U \times$ $\mathbb{R}^{m} \times \mathbb{R}^{n}$ with $\widehat{H^{\min }}(x, v, w, t)=(x, t v, w)$. Since the stratification induced

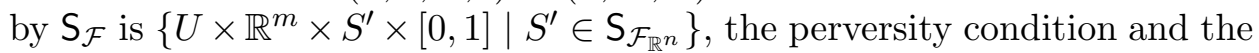
perverse degree are read on the $\mathbb{R}^{n}$-factor. So, the induced operator

$$
\left(\widetilde{H^{\min }}\right)^{*}: \Omega_{\bar{p}}^{*}\left(\tau^{-1}\left(T^{\min }\right) / \mathcal{F}\right) \rightarrow \Omega_{\bar{p}}^{*}\left(\tau^{-1}\left(T^{\min }\right) \times[0,1] / \mathcal{F} \times \mathcal{I}\right)
$$

is well defined. Integration along the $[0,1]$-factor does not involve $\mathbb{R}^{n}$. So, the integration operator

$$
K: \Omega_{\bar{p}}^{*}\left(\tau^{-1}\left(T^{\min }\right) / \mathcal{F}\right) \rightarrow \Omega_{\bar{p}}^{*-1}\left(\tau^{-1}\left(T^{\min }\right) / \mathcal{F}\right)
$$

given by $K(\omega)=\int_{0}^{1}\left(\widetilde{H^{\text {min }}}\right) * \omega$ is well defined. On the other hand, it satisfies the homotopy equality:

$$
\left.d \circ K+K \circ d=\left(\left(\widetilde{H^{\min }}\right)_{1}\right)^{*}-\left(\left(\widetilde{H^{\text {min }}}\right)_{0}\right)^{*}=\left(\widetilde{H^{\min }}\right)_{1}\right)^{*}-\mathrm{Id} .
$$

This gives $\left(\widetilde{\tau^{\min }}\right)^{*} \circ \widetilde{\iota}^{*}=\operatorname{Id}$. Since $\widetilde{\iota}^{*} \circ\left(\widetilde{\tau^{\min }}\right)^{*}=$ Id, we get $(18)$. 
(c) $\mathfrak{A}\left(\tau^{-1}\left(S-S^{\min }\right), \mathcal{F}\right)$ : The idea is to construct a $K$-invariant tubular neighborhood $\left(E, \nu, \widetilde{S}, \mathbb{R}^{n}\right)$ and a $K$-equivariant commutative diagram

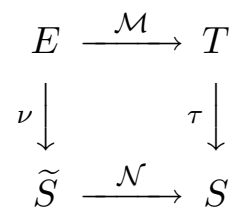

satisfying:

(i) $\mathcal{N}: \widetilde{S} \rightarrow S$ is the ot-blow-up of $S$ relative to the action $\Phi: K \times S \rightarrow S$ and depth $\mathrm{S}_{\widetilde{\Phi}}<\operatorname{depth} \mathrm{S}_{\Phi}$, where $\widetilde{\Phi}$ is the induced action of $K$ on $\widetilde{S}$.

(ii) The restrictions

$$
\begin{aligned}
& \mathcal{M}: \mathcal{M}^{-1} \tau^{-1}\left(S-S^{\mathrm{min}}\right) \rightarrow \tau^{-1}\left(S-S^{\mathrm{min}}\right), \\
& \mathcal{M}: \mathcal{M}^{-1} \tau^{-1}\left(T^{\mathrm{min}}-S^{\mathrm{min}}\right) \rightarrow \tau^{-1}\left(T^{\mathrm{min}}-S^{\mathrm{min}}\right)
\end{aligned}
$$

are trivial 2-coverings.

(iii) The invariant tubular neighborhood $\left(\mathcal{M}^{-1} \tau^{-1}\left(T^{\text {min }}\right), \nu, \mathcal{N}^{-1}\left(T^{\text {min }}\right)\right.$, $\left.\mathbb{R}^{n}\right)$ is $K$-equivariantly diffeomorphic to $\left(\tau^{-1}\left(D^{\text {min }}\right), \tau \times \operatorname{Id},\left(D^{\text {min }}\right) \times\right.$ ]$-1,1\left[, \mathbb{R}^{n}\right)$.

Conditions (ii) and (iii) give

$$
\begin{aligned}
\mathfrak{A}\left(\tau^{-1}\left(S-S^{\mathrm{min}}\right), \mathcal{F}\right) & \Leftrightarrow \mathfrak{A}\left(\mathcal{M}^{-1} \tau^{-1}\left(S-S^{\mathrm{min}}\right), \mathcal{E}\right), \\
\mathfrak{A}\left(\tau^{-1}\left(T^{\mathrm{min}}-S^{\mathrm{min}}\right), \mathcal{F}\right) & \Leftrightarrow \mathfrak{A}\left(\mathcal{M}^{-1} \tau^{-1}\left(T^{\mathrm{min}}-S^{\mathrm{min}}\right), \mathcal{E}\right), \\
\mathfrak{A}\left(\tau^{-1}\left(D^{\mathrm{min}}\right), \mathcal{F}\right) & \Leftrightarrow \mathfrak{A}\left(\mathcal{M}^{-1} \tau^{-1}\left(T^{\mathrm{min}}\right), \mathcal{E}\right),
\end{aligned}
$$

where $\mathcal{E}$ is the foliation induced by the action of $K$ on $E$.

The family $\left\{\mathcal{M}^{-1} \tau^{-1}\left(S-S^{\text {min }}\right), \mathcal{M}^{-1} \tau^{-1}\left(T^{\text {min }}\right)\right\}$ is a $K$-invariant open covering of $E$. Let $\alpha:\left[0,1\left[\rightarrow \mathbb{R}\right.\right.$ be as before. Set $f=\alpha \circ \varrho^{\min } \circ \mathcal{N} \circ \nu: E \rightarrow \mathbb{R}$, which is $K$-invariant and therefore $\mathcal{E}$-basic, where $\mathcal{E}$ is the foliation induced by the action of $K$ on $E$. Since $\operatorname{supp} f \subset \mathcal{M}^{-1} \tau^{-1}\left(T^{\text {min }}\right)$ and $\operatorname{supp}(1-f) \subset$ $\mathcal{M}^{-1} \tau^{-1}\left(S-S^{\text {min }}\right)$, the covering is basic. From 3.6 we get an exact MayerVietoris sequence

$$
\begin{aligned}
0 & \rightarrow \Omega_{\bar{p}}^{*}\left(\mathcal{M}^{-1} \tau^{-1}\left(T^{\text {min }}-S^{\text {min }}\right) / \mathcal{E}\right) \\
& \rightarrow \Omega_{\bar{p}}^{*}\left(\mathcal{M}^{-1} \tau^{-1}\left(S-S^{\text {min }}\right) / \mathcal{E}\right) \oplus \Omega_{\bar{p}}^{*}\left(\mathcal{M}^{-1} \tau^{-1}\left(T^{\text {min }}\right) / \mathcal{E}\right) \rightarrow \Omega_{\bar{p}}^{*}(E / \mathcal{E}) \rightarrow 0 .
\end{aligned}
$$

The Five Lemma and (20) give

$\mathfrak{A}\left(\tau^{-1}\left(T^{\mathrm{min}}-S^{\mathrm{min}}\right), \mathcal{F}\right) \& \mathfrak{A}\left(\tau^{-1}\left(D^{\mathrm{min}}\right), \mathcal{F}\right) \& \mathfrak{A}(E, \mathcal{E}) \Rightarrow \mathfrak{A}\left(\tau^{-1}\left(S-S^{\mathrm{min}}\right), \mathcal{F}\right)$.

We now check the three premises.

- $\mathfrak{A}\left(\tau^{-1}\left(T^{\text {min }}-S^{\text {min }}\right), \mathcal{F}\right)$ : This is condition (a).

- $\mathfrak{A}\left(\tau^{-1}\left(D^{\min }\right), \mathcal{F}\right)$ : By induction hypothesis since we have (17).

- $\mathfrak{A}(E, \mathcal{E})$ : By induction hypothesis since we have (8) by (i). 
It remains to construct (19). Consider the manifold

$$
\widetilde{S}=\left\{\left(D^{\min } \times\right]-1,1[) \amalg\left(\left(S-S^{\min }\right) \times\{-1,1\}\right)\right\} / \sim,
$$

where $(z, t) \sim\left(\nabla^{\min }(z,|t|), t /|t|\right)$, and the map $\mathcal{N}: \widetilde{S} \rightarrow S$ defined by

$$
\mathcal{N}(v)= \begin{cases}\nabla^{\min }(z,|t|) & \text { if } \left.v=(z, t) \in D^{\min } \times\right]-1,1[ \\ z & \text { if } v=(z, j) \in\left(S-S^{\min }\right) \times\{-1,1\} .\end{cases}
$$

This is the ot-blow-up $\mathcal{N}: \widetilde{S} \rightarrow S$ induced by the action $\Phi: K \times S \rightarrow S$.

Consider the manifold

$$
E=\left\{\left(\tau^{-1}\left(D^{\min }\right) \times\right]-1,1[) \amalg\left(\tau^{-1}\left(S-S^{\min }\right) \times\{-1,1\}\right)\right\} / \sim,
$$

where $(z, t) \sim\left(\widetilde{\nabla^{\min }}(z,|t|), t /|t|\right)$, the map $\mathcal{M}: E \rightarrow T$ defined by

$$
\mathcal{M}(v)= \begin{cases}\widetilde{\nabla^{\min }}(z,|t|) & \text { if } \left.v=(z, t) \in \tau^{-1}\left(D^{\min }\right) \times\right]-1,1[, \\ z & \text { if } v=(z, j) \in \tau^{-1}\left(S-S^{\min }\right) \times\{-1,1\},\end{cases}
$$

and the map $\nu: E \rightarrow \widetilde{S}$ defined by

$$
\nu(v)= \begin{cases}(\tau(z), t) & \text { if } \left.v=(z, t) \in \tau^{-1}\left(D^{\min }\right) \times\right]-1,1[ \\ (\tau(z), j) & \text { if } v=(z, j) \in \tau^{-1}\left(S-S^{\min }\right) \times\{-1,1\} .\end{cases}
$$

Since $\nabla^{\mathrm{min}}$ and $\widetilde{\nabla^{\mathrm{min}}}$ are $K$-equivariant embeddings, $\widetilde{S}$ and $E$ are $K$-manifolds. The maps $\mathcal{N}$ and $\mathcal{M}$ are $K$-equivariant continuous maps. Since $\tau$ is $K$-equivariant, so is $\nu$. The diagram (19) is clearly commutative.

$\left(E, \nu, \widetilde{S}, \mathbb{R}^{n}\right)$ is a tubular neighborhood since $\left(\tau^{-1}\left(S-S^{\mathrm{min}}\right), \tau, S-S^{\mathrm{min}}\right.$, $\left.\mathbb{R}^{n}\right)$ and $\left(\tau^{-1}\left(D^{\min }\right) \times\right]-1,1\left[, \tau \times \operatorname{Id}_{-1,1}, D^{\min } \times\right]-1,1\left[, \mathbb{R}^{n}\right)$ are compatible tubular neighborhoods. It remains to verify properties (i)-(iii).

(i) By construction.

(ii) By construction $\mathcal{M}^{-1} \tau^{-1}\left(S-S^{\text {min }}\right)=\tau^{-1}\left(S-S^{\text {min }}\right) \times\{-1,1\}$ and $\mathcal{M}$ is the projection on the first factor.

(iii) By construction $\left.\mathcal{N}^{-1}\left(T^{\text {min }}\right)=D^{\text {min }} \times\right]-1,1\left[, \mathcal{M}^{-1} \tau^{-1}\left(T^{\text {min }}\right)=\right.$ $\left.\tau^{-1}\left(D^{\min }\right) \times\right]-1,1\left[\right.$ and $\nu$ becomes $\tau \times \operatorname{Id}_{-1,1}[$.

This ends the proof.

The first main result of this section is the following

4.2.2. TheOREM. The BIC of the foliation determined by an isometric action of an abelian Lie group on a compact manifold is finite-dimensional.

Proof. Given a conical foliated manifold $(N \mathcal{N})$ we consider the statement

$\mathfrak{A}(N, \mathcal{N})=$ "The BIC $\mathbb{H}_{\bar{p}}^{*}(N / \mathcal{N})$ is finite-dimensional for any perversity $\bar{p}$.

Consider an isometric action $\Phi: G \times M \rightarrow M$ of an abelian Lie group $G$ on a compact manifold $M$. This is equivalent to saying that the action 
is tame. We denote by $\mathcal{F}$ the induced conical foliation. Suppose that $G$ is connected and dense in the (connected) tamer group $K$. We prove $\mathfrak{A}(M, \mathcal{F})$ by induction on depth $S_{\mathcal{F}}$.

FIRST STEP: $\operatorname{depth} S_{\mathcal{F}}=0$. The foliation $\mathcal{F}$ is a (regular) riemannian foliation (cf. [12]) and the BIC is just the basic cohomology (cf. 3.4.1(a)) The result comes directly from [7].

Induction step: The family $\left\{M-S_{\min }, T_{\min }\right\}$ is a $K$-invariant basic open covering of $M$ (cf. proof of Proposition 4.1). From 3.6 we get an exact Mayer-Vietoris sequence

$$
\begin{aligned}
0 \rightarrow \Omega_{\bar{p}}^{*}\left(T_{\min }-S_{\min } / \mathcal{F}\right) \rightarrow \Omega_{\bar{p}}^{*}\left(M-S_{\min } / \mathcal{F}\right) \oplus \Omega_{\bar{p}}^{*}\left(T_{\min } / \mathcal{F}\right) & \\
& \rightarrow \Omega_{\bar{p}}^{*}(M / \mathcal{F}) \rightarrow 0 .
\end{aligned}
$$

The Five Lemma gives

$$
\mathfrak{A}\left(T_{\min }-S_{\min }, \mathcal{F}\right) \& \mathfrak{A}\left(T_{\min }, \mathcal{F}\right) \& \mathfrak{A}\left(M-S_{\text {min }}, \mathcal{F}\right) \Rightarrow \mathfrak{A}(M, \mathcal{F}) .
$$

We now check the three premises.

(a) $\mathfrak{A}\left(T_{\min }-S_{\min }, \mathcal{F}\right)$ : Since $\nabla^{\min }$ is a $K$-equivariant diffeomorphism we have

$$
\mathfrak{A}\left(T_{\min }-S_{\text {min }}, \mathcal{F}\right) \Leftrightarrow \mathfrak{A}\left(D^{\min } \times\right]-1,1[, \mathcal{F} \times \mathcal{I}) \stackrel{3.5 .1}{\Longleftrightarrow} \mathfrak{A}\left(D^{\min }, \mathcal{F}\right),
$$

which is true since $\operatorname{depth}\left(D^{\min }, \mathcal{F}\right)<\operatorname{depth} \mathrm{S}_{\mathcal{F}}($ cf. 2.5).

(b) $\mathfrak{A}\left(T_{\min }, \mathcal{F}\right)$ : If we prove $\mathfrak{A}\left(\mathbb{R}^{n}, \mathcal{F}_{\mathbb{R}^{n}}\right)$ for the slice of a tubular neighborhood $\left(T, S, \tau, \mathbb{R}^{n}\right)$, then it suffices to apply Proposition 4.2.1. Recall that $\mathcal{F}_{\mathbb{R}^{n}}$ is defined by an orthogonal action $\Theta: G \cap H \times \mathbb{R}^{n} \rightarrow \mathbb{R}^{n}$ such that $\Theta: G \cap H \times \mathbb{S}^{n-1} \rightarrow \mathbb{S}^{n-1}$ is a tame action without fixed points defining $\mathcal{G}$ and satisfying $\left(\mathbb{R}^{n}, \mathcal{F}_{\mathbb{R}^{n}}\right)=\left(c \mathbb{S}^{n-1}, c \mathcal{G}_{S}\right)$. We have $H_{\bar{p}}^{*}\left(\mathbb{R}^{n} / \mathcal{F}_{\mathbb{R}^{n}}\right) \stackrel{3.5 .2}{\cong}$ $H_{\bar{p}}^{\leq \bar{p}(\vartheta)}\left(\mathbb{S}^{n-1} / \mathcal{G}_{S}\right)$, the truncated cohomology, which is finite-dimensional since depth $\mathrm{S}_{\mathcal{G}_{S}}<\operatorname{depth} \mathrm{S}_{\mathcal{F}}$ implies $\mathfrak{A}\left(\mathbb{S}^{n-1}, \mathcal{G}_{S}\right)$.

(c) $\mathfrak{A}\left(M-S_{\min }, \mathcal{F}\right)$ : The family $\left\{\mathcal{L}^{-1}\left(M-S_{\min }\right), \mathcal{L}^{-1}\left(T_{\min }\right)\right\}$ is a $K-$ invariant open covering of $M$. Take $\alpha:[0,1[\rightarrow \mathbb{R}$ as before. Set $f=\alpha \circ$ $\varrho_{\text {min }} \circ \mathcal{L}: \widehat{M} \rightarrow \mathbb{R}$, which is $K$-invariant map and therefore $\widehat{\mathcal{F}}$-basic. Since $\operatorname{supp} f \subset \mathcal{L}^{-1}\left(T_{\text {min }}\right)$ and $\operatorname{supp}(1-f) \subset \mathcal{L}^{-1}\left(M-S_{\text {min }}\right)$, the covering is basic. From 3.6 we get an exact Mayer-Vietoris sequence

$$
\begin{aligned}
0 \rightarrow \Omega_{\bar{p}}^{*}\left(\mathcal{L}^{-1}\left(T_{\text {min }}-S_{\text {min }}\right) / \widehat{\mathcal{F}}\right) & \\
\rightarrow & \Omega_{\bar{p}}^{*}\left(\mathcal{L}^{-1}\left(M-S_{\text {min }}\right) / \widehat{\mathcal{F}}\right) \oplus \Omega_{\bar{p}}^{*}\left(\mathcal{L}^{-1}\left(T_{\text {min }}\right) / \widehat{\mathcal{F}}\right) \rightarrow \Omega_{\bar{p}}^{*}(\widehat{M} / \widehat{\mathcal{F}}) \rightarrow 0 .
\end{aligned}
$$

Recall that, by $2.5, \mathcal{L}: \mathcal{L}^{-1}\left(M-S_{\text {min }}\right) \rightarrow\left(M-S_{\text {min }}\right)$ is a $K$-equivariant smooth trivial 2-covering. So, $\mathfrak{A}\left(\mathcal{L}^{-1}\left(M-S_{\min }\right), \widehat{\mathcal{F}}\right) \Leftrightarrow \mathfrak{A}\left(M-S_{\min }, \mathcal{F}\right)$. Now, the Five Lemma gives

$\mathfrak{A}\left(\mathcal{L}^{-1}\left(T_{\text {min }}-S_{\text {min }}\right), \widehat{\mathcal{F}}\right) \& \mathfrak{A}\left(\mathcal{L}^{-1}\left(T_{\text {min }}\right), \widehat{\mathcal{F}}\right) \& \mathfrak{A}(\widehat{M}, \widehat{\mathcal{F}}) \Rightarrow \mathfrak{A}\left(M-S_{\text {min }}, \mathcal{F}\right)$. 
We now check the three premises.

- $\mathfrak{A}\left(\mathcal{L}^{-1}\left(T_{\min }-S_{\text {min }}\right), \widehat{\mathcal{F}}\right):$ Since $\mathcal{L}^{-1}\left(T_{\min }-S_{\text {min }}\right)$ is $K$-diffeomorphic to two copies of $T_{\min }-S_{\min }$ (cf. 2.4), we have

$$
\mathfrak{A}\left(\mathcal{L}^{-1}\left(T_{\min }-S_{\min }\right), \widehat{\mathcal{F}}\right) \Leftrightarrow \mathfrak{A}\left(T_{\min }-S_{\min }, \mathcal{F}\right) .
$$

Now we apply (a).

- $\mathfrak{A}\left(\mathcal{L}^{-1}\left(T_{\min }\right), \widehat{\mathcal{F}}\right)$ : From 2.4 we know that $\mathcal{L}^{-1}\left(T_{\text {min }}\right)$ is $K$-diffeomorphic to $\left.D_{\min } \times\right]-1,1[$. Now we proceed as in (a).

- $\mathfrak{A}(\widehat{M}, \widehat{\mathcal{F}}):$ Because depth $\mathrm{S}_{\widehat{\mathcal{F}}}<\operatorname{depth} \mathrm{S}_{\mathcal{F}}$ (cf. 2.4).

4.3. Poincaré duality. In this section we prove that the $\mathrm{BIC}$ of a conical foliation $\mathcal{F}$ defined on an oriented manifold $M$ and determined by a tame action satisfies the Poincaré duality:

$$
\mathbb{I H}_{\bar{p}}^{*}(M / \mathcal{F}) \cong \mathbb{I} H_{\bar{q}, \mathrm{c}}^{\ell-*}(M / \mathcal{F}) .
$$

Here $\ell\left(\right.$ or $\left.\ell_{M}\right)$ is the codimension of the foliation $\mathcal{F}$. The two perversities $\bar{p}$ and $\bar{q}$ are complementary, that is, $\bar{p}+\bar{q}=\bar{t}$.

The proof follows the route of 4.2 , but first we define a morphism $P_{M}$ giving (21); it depends on the notion of a tangent volume form.

4.3.1. Tangent volume form. For the definition of the pairing $P_{M}$ we need a volume form tangent to the leaves of $\mathcal{F}$.

Let $\Phi: G \times M \rightarrow M$ be a tame action defining $\mathcal{F}$. We can choose the group $G$ to be connected and the action $\Phi$ effective; so, $b=\operatorname{dim} G=\operatorname{dim} \mathcal{F}$. We also fix a basis $\left\{u_{1}, \ldots, u_{b}\right\}$ of the Lie algebra $\mathfrak{g}$ of $G$. The associated fundamental vector fields on $M$ are denoted by $\left\{V_{1}, \ldots, V_{b}\right\}$.

A tangent volume form of $(M, \mathcal{F})$ is a $G$-invariant differential form $\eta \in$ $\Pi_{\mathcal{F}}^{b}(M)$ satisfying

$$
\eta\left(V_{1}, \ldots, V_{b}\right)=1
$$

Notice that $d \eta\left(V_{1}, \ldots, V_{b},-\right)=0$.

4.3.2. Proposition. Let $K$ be a tamer group of $G$. There exists a $K$ invariant tangent volume form $\eta$ of $(M, \mathcal{F})$ with the following properties:

(a) For each $\omega \in \Omega_{\frac{\ell}{t}}^{\ell}(M / \mathcal{F})$, we have $\omega \wedge \eta=\omega \wedge \eta^{\prime}$ for any tangent volume form $\eta^{\prime}$.

(b) For each $\omega \in \Omega_{\bar{t}}^{\ell-1}(M / \mathcal{F})$ the product $\omega \wedge d \eta$ is 0 .

(c) For each $\omega \in \Omega_{\bar{t}, \mathrm{c}}^{\ell}(M / \mathcal{F})$ the integral $\int_{R_{\mathcal{F}}} \omega \wedge \eta$ is finite.

(d) For each $\omega \in \Omega_{\bar{t}, \mathrm{c}}^{\ell-1}(M / \mathcal{F})$ the integral $\int_{R_{\mathcal{F}}} d(\omega \wedge \eta)$ is 0 . 
Proof. We first prove the following statement by induction on depth $\mathrm{S}_{\mathcal{F}}$ :

For each $p \in \mathbb{N}$ there exists a $K$-invariant differential form

$\eta \in \Pi_{\mathcal{F} \times \mathcal{I}}^{b}\left(M \times\left[0,1\left[^{p}\right)\right.\right.$ satisfying

$$
\eta\left(\left(V_{1}, 0\right), \ldots,\left(V_{b}, 0\right)\right)=1 .
$$

The desired $\eta$ is obtained by taking $p=0$.

If depth $S_{\mathcal{F}}=0$ we define $\eta_{0}$ on the orbits of $\Phi$ by (23) and we extend it to a differential form in $\Omega^{b}\left(M \times\left[0,1\left[^{p}\right)\right.\right.$. The differential form $\eta=\int_{K} k^{*} \eta_{0}$ is $K$-invariant, lives in $\Pi_{\mathcal{F} \times \mathcal{I}}^{b}\left(M \times\left[0,1\left[^{p}\right)\right.\right.$ and satisfies (23) since each $k$ is a $K$-equivariant diffeomorphism.

Consider now the case depth $S_{\mathcal{F}}>0$. By induction hypothesis there exists a $K$-invariant differential form $\eta_{0} \in \Pi_{\widehat{\mathcal{F}}}^{b}\left(\widehat{M} \times\left[0,1\left[^{p}\right)\right.\right.$ satisfying (23). Associated to Molino's blow-up we have the $K$-equivariant embedding $S: M-$ $S_{\text {min }} \rightarrow \mathcal{L}^{-1}\left(M-S_{\text {min }}\right)$ defined by $\sigma(z)=(z, 1)$. The differential form $\eta=\left(\sigma \times \operatorname{Id}_{\left[0,1\left[^{p}\right.\right.}\right)^{*} \eta_{0}$ belongs to $\Omega^{b}\left(R_{\mathcal{F}} \times\left[0,1\left[^{p}\right)\right.\right.$. It is $K$-invariant and satisfies (23) since $\sigma$ is a $K$-equivariant embedding. It remains to prove that $\eta \in \Pi_{\mathcal{F} \times \mathcal{I}}^{b}\left(M \times\left[0,1\left[^{p}\right)\right.\right.$, which is a local property. So, we can assume that $M$ is a tubular neighborhood $T$ of a singular stratum of $\mathrm{S}_{\mathcal{F}}$ and prove $\left(\nabla \times \operatorname{Id}_{\left[0,1\left[^{p}\right.\right.}\right)^{*} \eta \in \Pi_{\mathcal{F} \times \mathcal{I}}^{b}\left(D \times\left[0,1\left[^{p+1}\right)\right.\right.$ (cf. 3.1.1(e)). This is the case since $\sigma \circ \nabla: D \times] 0,1[\rightarrow D \times]-1,1[$ is just the inclusion and $\eta_{0} \in \Pi_{\mathcal{F} \times \mathcal{I}}^{b}\left(D \times\left[0,1\left[{ }^{p+1}\right)\right.\right.$.

(a) Let $\eta^{\prime}$ be another tangent volume form associated to $\mathcal{F}$ through $\Phi$ and $\left\{u_{1}, \ldots, u_{b}\right\}$. For degree reasons it suffices to prove $i_{V_{1}} \cdots i_{V_{b}}(\omega \wedge \eta)=$ $i_{V_{1}} \cdots i_{V_{b}}\left(\omega \wedge \eta^{\prime}\right)$. Since $\omega$ is a basic form, we have

$$
\begin{aligned}
i_{V_{1}} \cdots i_{V_{b}}(\omega \wedge \eta) & =(-1)^{\ell b} \omega \wedge\left(i_{V_{1}} \cdots i_{V_{b}} \eta\right)=(-1)^{\ell b} \omega \wedge\left(i_{V_{1}} \cdots i_{V_{b}} \eta^{\prime}\right) \\
& =i_{V_{1}} \cdots i_{V_{b}}\left(\omega \wedge \eta^{\prime}\right) .
\end{aligned}
$$

(b) For degree reasons it suffices to prove that $i_{V_{1}} \cdots i_{V_{b}}(\omega \wedge d \eta)=0$. Indeed, since $\omega$ is a basic form, $i_{V_{1}} \cdots i_{V_{b}}(\omega \wedge d \eta)=(-1)^{\ell b} \omega \wedge i_{V_{1}} \cdots i_{V_{b}} d \eta=0$.

(c) It suffices to prove that $\int_{R_{\mathcal{F}} \times\left[0,1\left[^{p}\right.\right.} \gamma<\infty$ where $\gamma \in \Pi_{\mathcal{F} \times \mathcal{I}}^{m+p}\left(M \times\left[0,1\left[^{p}\right)\right.\right.$ with compact support. We proceed by induction on $\operatorname{depth} S_{\mathcal{F}}$. When the foliation is regular the result is clear. In the general case we know that the result is true for $M-S_{\min } \times\left[0,1\left[^{p}\right.\right.$ and $\left(T_{\min }-S_{\min }\right) \times\left[0,1\left[{ }^{p}\right.\right.$. It remains to consider $T_{\min } \times\left[0,1\left[^{p}\right.\right.$. From 3.4.1(d) we know that we can identify the perverse forms on $T_{\min } \times\left[0,1\left[^{p}\right.\right.$ with the perverse forms on $D_{\min } \times\left[0,1\left[^{p+1}\right.\right.$ through the map

$$
\nabla_{\min } \times \operatorname{Id}_{\left[0,1\left[^{p}\right.\right.}: D_{\min } \times\left[0,1\left[\times\left[0,1\left[^{p} \equiv D_{\min } \times\left[0,1\left[^{p+1} \rightarrow T_{\min } \times\left[0,1\left[^{p} .\right.\right.\right.\right.\right.\right.\right.\right.
$$

Since this map is a diffeomorphism between $\left.D_{\min } \times\right] 0,1\left[\times\left[0,1\left[^{p}\right.\right.\right.$ and 
$\left(T_{\min }-S_{\min }\right) \times\left[0,1\left[^{p}\right.\right.$, we have

$$
\int_{R_{\mathcal{F}_{T_{\min }}} \times\left[0,1\left[^{p}\right.\right.} \gamma=\int_{R_{\left.\mathcal{F}_{D_{\min }} \times\right], 1\left[\times\left[0,1\left[p^{p}\right.\right.\right.}} \gamma=\int_{R_{\mathcal{F}_{D_{\min }} \times\left[0,1\left[\times\left[0,1\left[p^{p}\right.\right.\right.\right.}} \gamma=\int_{R_{\mathcal{F}_{D_{\min }} \times\left[0,1\left[\left[^{p+1}\right.\right.\right.}} \gamma .
$$

The induction hypothesis shows that the last integral is finite.

(d) Since $\operatorname{supp} \omega$ is compact, it suffices to prove $\int_{U \cap R_{\mathcal{F}}} d(\omega \wedge \eta)=0$ where $(U, \varphi)$ is a conical chart of $\mathcal{F}$ and $\omega \in \Omega_{\bar{t}, \mathrm{c}}^{\ell-1}(U / \mathcal{F})$ with $\operatorname{supp} \omega \subset U$. We have

$$
\begin{aligned}
\int_{U \cap R_{\mathcal{F}}} d(\omega \wedge \eta) & =\int_{\left.\mathbb{R}^{m-n-1} \times R_{\mathcal{G}} \times\right] 0,1[} d\left(P_{\varphi}^{*} \omega \wedge P_{\varphi}^{*} \eta\right) \\
& \stackrel{(10)}{=} \int_{\mathbb{R}^{m-n-1} \times R_{\mathcal{G}} \times[0,1[} d\left(\omega_{\varphi} \wedge \eta_{\varphi}\right) \\
& \stackrel{\text { Stokes }}{=} \int_{\mathbb{R}^{m-n-1} \times R_{\mathcal{G}} \times\{0\}} \omega_{\varphi} \wedge \eta_{\varphi} \stackrel{3.4 .1(\mathrm{c})}{=} 0 .
\end{aligned}
$$

4.3.3. The pairing. Let $\eta$ be a tangent volume form. Consider the map

$$
P_{M}: \Omega_{\bar{p}}^{*}(M / \mathcal{F}) \times \Omega_{\bar{q}, c}^{\ell-*}(M / \mathcal{F}) \rightarrow \mathbb{R}, \quad P_{M}(\alpha, \beta)=\int_{R_{\mathcal{F}}} \alpha \wedge \beta \wedge \eta .
$$

Notice that $R_{\mathcal{F}} \subset M$ is an oriented manifold. By Proposition 4.3.2(c) the integral is well defined. This map depends on the action $\Phi: G \times M \rightarrow M$ and on the choice of the basis $\left\{u_{1}, \ldots, u_{b}\right\}$ of $\mathfrak{g}$ (cf. Proposition 4.3.2(a)).

The pairing is the induced map

$$
P_{M}: H_{\bar{p}}^{*}(M / \mathcal{F}) \times H_{\bar{q}, \mathrm{c}}^{\ell-*}(M / \mathcal{F}) \rightarrow \mathbb{R}
$$

defined by

$$
P_{M}([\alpha],[\beta])=\int_{R_{\mathcal{F}}} \alpha \wedge \beta \wedge \eta
$$

This map is well defined (cf. Proposition 4.3.2(b) and (d)). The Poincaré duality asserts that $P_{M}$ is a non-degenerate pairing, that is, the map

$$
P_{M}: \mathbb{H}_{\bar{p}}^{*}(M / \mathcal{F}) \rightarrow \operatorname{Hom}\left(\mathbb{H}_{\bar{q}, \mathrm{c}}^{\ell-*}(M / \mathcal{F}), \mathbb{R}\right)
$$

defined by

$$
P_{M}([\alpha])([\beta])=\int_{R_{\mathcal{F}}} \alpha \wedge \beta \wedge \eta
$$

is an isomorphism.

The first step to prove the Poincaré duality is

4.3.4. Proposition. Consider a twisted product $K \times_{H} \mathbb{R}^{n}$ as in 2.2 . Suppose that the action $\Phi: G \times\left(K \times_{H} \mathbb{R}^{n}\right) \rightarrow K \times_{H} \mathbb{R}^{n}$ is effective. Fix a 
basis of $\mathfrak{k}$ as in 3.8.3. The BIC of $\left(K \times_{H} \mathbb{R}^{n}, \mathcal{F}_{\mathrm{tw}}\right)$ satisfies the Poincaré duality whenever the BIC of its slice $\left(\mathbb{R}^{n}, \mathcal{F}_{\mathbb{R}^{n}}\right)$ does.

Proof. Since the action $\Phi: G \times\left(K \times_{H} \mathbb{R}^{n}\right) \rightarrow K \times_{H} \mathbb{R}^{n}$ defining $\mathcal{F}_{\text {tw }}$ is effective, so is the action $\Theta:(G \cap H) \times \mathbb{R}^{n} \rightarrow \mathbb{R}^{n}$ defining $\mathcal{F}_{\mathbb{R}^{n}}$. Hence the proposition makes sense. We now proceed in two steps.

(a) Construction of the tangent volume form. Consider a tangent volume form $\eta_{0} \in\left(\Pi_{\mathcal{F}_{\mathbb{R}^{n}}}^{b-a}\left(\mathbb{R}^{n}\right)\right)^{H}$ of the slice $\left(\mathbb{R}^{n}, \mathcal{F}_{\mathbb{R}^{n}}\right)$. We construct the tangent volume form $\eta$ of the twisted product in terms of $\eta_{0}$.

Put $\eta=\nabla^{-1}\left(\gamma_{1} \wedge \cdots \wedge \gamma_{a} \wedge \eta_{0}\right)=R^{-*}\left(\gamma_{1} \wedge \cdots \wedge \gamma_{a} \wedge \Delta^{\prime}\left(\eta_{0}\right)\right)$. It belongs to $\left(R_{\mathcal{F}}^{*}\left(K \times_{H} \mathbb{R}^{n}\right)\right)^{K}$ (cf. 3.8.5). We now prove (22). Since $R_{*} X_{i}=V_{i}$ for $i \in\{1, \ldots, f\}$, we have

$$
\begin{aligned}
& \eta\left(V_{1}, \ldots, V_{b}\right)=\left(\gamma_{1} \wedge \cdots \wedge \gamma_{a} \wedge \Delta^{\prime}\left(\eta_{0}\right)\right)\left(X_{1}, \ldots, X_{b}\right)=\Delta^{\prime}\left(\eta_{0}\right)\left(X_{a+1}, \ldots, X_{b}\right) \\
& =\left(\eta_{0}+\sum_{a+1 \leq i_{1}<\cdots<i_{\ell} \leq c} \gamma_{i_{1}} \wedge \cdots \wedge \gamma_{i_{\ell}} \wedge\left(i_{W_{i_{\ell}}} \cdots i_{W_{i_{1}}} \eta_{0}\right)\right)\left(X_{a+1}, \ldots, X_{b}\right) \\
& =i_{W_{b}} \cdots i_{W_{a+1}} \eta_{0}=1
\end{aligned}
$$

from (22) for $\eta_{0}$. We conclude that $\eta$ is a tangent volume form of the twisted product. Recall that the pairing $P_{M}$ can be defined using this form $\eta$ (cf. Proposition 4.3.2(a)).

(b) Poincaré duality. Consider now two complementary perversities $\bar{p}$ and $\bar{q}$ on $K \times_{H} \mathbb{R}^{n}$. The induced perversities $\bar{p}$ and $\bar{q}$ on $\mathbb{R}^{n}$ are also complementary: for each stratum $S \in \mathrm{S}_{\mathcal{F}_{\mathbb{R}^{n}}}$, we have

$$
\begin{aligned}
\bar{p}(S)+\bar{q}(S) & =\bar{p}\left(K \times_{H} S\right)+\bar{q}\left(K \times_{H} S\right)=\bar{t}\left(K \times_{H} S\right) \\
& =\operatorname{dim} K \times_{H} \mathbb{R}^{n}-\operatorname{dim} K \times_{H} S-\operatorname{dim} G_{K \times_{H} S}-2 \\
& =\operatorname{dim} \mathbb{R}^{n}-\operatorname{dim} S-\operatorname{dim}(G \cap H)_{S}-2=\bar{t}(S) .
\end{aligned}
$$

By hypothesis the pairing $P_{\mathbb{R}^{n}}: H_{\bar{p}}^{*}\left(\mathbb{R}^{n} / \mathcal{F}_{\mathbb{R}^{n}}\right) \times \mathbb{H}_{\bar{q}, c}^{\ell_{\mathbb{R}^{n}-*}}\left(\mathbb{R}^{n} / \mathcal{F}_{\mathbb{R}^{n}}\right) \rightarrow \mathbb{R}$ is non-degenerate. Since $\eta_{0}$ is $H$-invariant, the pairing $P_{\mathbb{R}^{n}}:\left(\mathbb{H}_{\bar{p}}^{*}\left(\mathbb{R}^{n} / \mathcal{F}_{\mathbb{R}^{n}}\right)\right)^{H} \times$ $\left(\mathbb{H}_{\bar{q}, \mathrm{c}}^{\ell \mathbb{R}^{n-*}}\left(\mathbb{R}^{n} / \mathcal{F}_{\mathbb{R}^{n}}\right)\right)^{H} \rightarrow \mathbb{R}$ is also non-degenerate. On the other hand, it is clear that the pairing $P: \bigwedge^{*}\left(\gamma_{c+1}, \ldots, \gamma_{f}\right) \times \bigwedge^{f-c-*}\left(\gamma_{c+1}, \ldots, \gamma_{f}\right) \rightarrow \mathbb{R}$ defined by

$$
P\left(\gamma_{i_{1}} \wedge \cdots \wedge \gamma_{i_{u}}, \gamma_{j_{1}} \wedge \cdots \wedge \gamma_{j_{f-c-u}}\right)=\int_{K / H} \gamma_{i_{1}} \wedge \cdots \wedge \gamma_{i_{u}} \wedge \gamma_{j_{1}} \wedge \cdots \wedge \gamma_{j_{f-c-u}}
$$

is non-degenerate. Notice the equality $\ell_{K \times_{H} \mathbb{R}^{n}}=f+a+n-c-b=\ell_{\mathbb{R}^{n}}+f-c$.

We prove that the pairing

$$
P_{K \times_{H} \mathbb{R}^{n}}: \mathbb{H}_{\bar{p}}^{*}\left(K \times_{H} \mathbb{R}^{n}\right) \times H_{\bar{q}, c}^{\ell_{K \times} \mathbb{R}^{\mathbb{R}^{n-*}}}\left(K \times_{H} \mathbb{R}^{n}\right) \rightarrow \mathbb{R}
$$

is non-degenerate. We know, from Proposition 3.8.4, that this is the case if 
the following diagram commutes (up to sign):

$$
\begin{aligned}
& \left(\bigwedge^{*}\left(\gamma_{c+1}, \ldots, \gamma_{f}\right) \otimes\left(H_{\bar{p}}^{*}\left(\mathbb{R}^{n} / \mathcal{F}_{\mathbb{R}^{n}}\right)\right)^{H}\right) \\
& \times\left(\bigwedge^{f-c-*}\left(\gamma_{c+1}, \ldots, \gamma_{f}\right) \otimes\left(H_{\bar{p}}^{\ell_{\mathbb{R}^{n-*}}}\left(\mathbb{R}^{n} / \mathcal{F}_{\mathbb{R}^{n}}\right)\right)^{H}\right) \stackrel{P \otimes P_{\mathbb{R}^{n}}}{\longrightarrow} \mathbb{R} \\
& \nabla^{-*} \times \nabla^{-*} \downarrow \\
& I_{\bar{p}}^{*}\left(K \times_{H} \mathbb{R}^{n}\right) \times \mathbb{H}_{\bar{q}, \mathrm{c}}^{\ell_{K \times} \mathbb{R}^{n-*}}\left(K \times_{H} \mathbb{R}^{n}\right) \quad \stackrel{P_{K \times} \mathbb{R}^{n}}{\longrightarrow} \mathbb{R}
\end{aligned}
$$

Let us see that. For each $[\alpha] \in\left(H_{\bar{p}}^{i}\left(\mathbb{R}^{n} / \mathcal{F}_{\mathbb{R}^{n}}\right)\right)^{H}$ and each $[\beta] \in$ $\left(\mathbb{H}_{\bar{p}}^{\ell_{0}-i}\left(\mathbb{R}^{n} / \mathcal{F}_{\mathbb{R}^{n}}\right)\right)^{H}$, we have, for degree reasons,

$$
\begin{aligned}
P_{K \times{ }_{H} \mathbb{R}^{n}}\left(\nabla^{-1} \times \nabla^{-1}\right)\left(\gamma_{i_{1}} \wedge \cdots \wedge \gamma_{i_{u}} \otimes[\alpha], \gamma_{j_{1}} \wedge \cdots \wedge \gamma_{j_{f-c-u}} \otimes[\beta]\right) \\
=\int_{K \times_{H} \mathbb{R}^{n}} R^{-*}\left(\gamma_{i_{1}} \wedge \cdots \wedge \gamma_{i_{u}} \wedge \alpha \wedge \gamma_{j_{1}} \wedge \cdots \wedge \gamma_{j_{f-c-u}} \wedge \beta \wedge \gamma_{1} \wedge \cdots \wedge \gamma_{a} \wedge \eta_{0}\right) \\
=\int_{K \times \mathbb{R}^{n}} \gamma_{i_{1}} \wedge \cdots \wedge \gamma_{i_{u}} \wedge \alpha \wedge \gamma_{j_{1}} \wedge \cdots \wedge \gamma_{j_{f-c-u}} \wedge \beta \wedge \gamma_{1} \wedge \cdots \wedge \gamma_{a} \wedge \eta_{0} \wedge \gamma_{a+1} \wedge \cdots \wedge \gamma_{c} \\
=\int_{K} \gamma_{1} \wedge \cdots \wedge \gamma_{c} \wedge \gamma_{i_{1}} \wedge \cdots \wedge \gamma_{i_{u}} \wedge \gamma_{j_{1}} \wedge \cdots \wedge \gamma_{j_{f-c-u}} \cdot \int_{\mathbb{R}^{n}} \alpha \wedge \beta \wedge \eta_{0} \\
=P\left(\gamma_{i_{1}} \wedge \cdots \wedge \gamma_{i_{u}}, \gamma_{j_{1}} \wedge \cdots \wedge \gamma_{j_{f-c-u}}\right) \cdot P_{\mathbb{R}^{n}}([\alpha],[\beta]) \\
=\left(P \otimes P_{\mathbb{R}^{n}}\right)\left(\gamma_{i_{1}} \wedge \cdots \wedge \gamma_{i_{u}} \otimes[\alpha], \gamma_{j_{1}} \wedge \cdots \wedge \gamma_{j_{f-c-u}} \otimes[\beta]\right),
\end{aligned}
$$

up to sign.

We now study the Poincaré duality of the tubular neighborhoods of the strata of $\mathrm{S}_{\mathcal{F}}$ (cf. 2.3). Consider a $K$-invariant tubular neighborhood $\left(T, \tau, S, \mathbb{R}^{n}\right)$ of a singular stratum $S$, where $K$ is a tamer group of $G$ with $K=\bar{G}$. Let $\left(\mathbb{R}^{n}, \mathcal{F}_{\mathbb{R}^{n}}\right)$ be the slice of the tubular neighborhood. The foliation $\mathcal{F}_{\mathbb{R}^{n}}$ is defined by an effective tame action $\Theta:\left(G_{S}\right)_{0} \times \mathbb{R}^{n} \rightarrow \mathbb{R}^{n}$. We fix a basis $\left\{u_{1}, \ldots, u_{b}\right\}$ of $\mathfrak{g}$ and suppose that $\left\{u_{a+1}, \ldots, u_{b}\right\}$ is a basis of the Lie algebra of $G_{S}$.

4.3.5. Proposition. Under the above conditions, if the BIC of the slice $\left(\mathbb{R}^{n}, \mathcal{F}_{\mathbb{R}^{n}}\right)$ satisfies the Poincaré duality then so does the BIC of the tube $(T, \mathcal{F})$.

Proof. The proof is the same of that of Proposition 4.2.1 with $\mathfrak{A}(T, \mathcal{F})=$ "The pairing $P_{T}: \mathbb{H}_{\bar{p}}^{*}(T / \mathcal{F}) \rightarrow \mathbb{H}_{\bar{q}}^{\ell-*}(T / \mathcal{F})$ is non-degenerate for any two complementary perversities $\bar{p}$ and $\bar{q}$ ".

We consider the orbit type stratification of $S$ induced by the action $\Phi_{S}: K \times S \rightarrow S$ of $\Phi$. We proceed by induction on the depth of this stratification. By using the Mayer-Vietoris technics of 3.6 and 3.7 we can suppose that $\Phi_{S}$ defines a fiber bundle $\pi: S \rightarrow S / K$ whose fiber is $K / H$. Considering 
a good covering of $S / K$, the Mayer-Vietoris procedure leads us to the case $S / K=$ point, that is, to the case where $T$ is the twisted product $K \times_{H} \mathbb{R}^{n}$. Here, we apply Proposition 4.3.4.

The second main result of this section is the following

4.3.6. TheOREM. The BIC of the foliation determined by an isometric action of an abelian Lie group on an oriented compact manifold satisfies the Poincaré duality.

Proof. In fact, we prove the result for a foliation $\mathcal{F}$ determined by a tame action $\Phi: G \times M \rightarrow M$ on an oriented manifold, not necessarily compact. We can suppose that $G$ is connected and that the action $\Phi$ is effective. We fix a tamer group $K$ in which $G$ is dense.

The proof is the same as that of Theorem 4.2.2 with $\mathfrak{A}(M, \mathcal{F})=$ "The pairing $P_{T}: \mathbb{H}_{\bar{p}}^{*}(M / \mathcal{F}) \rightarrow \mathbb{H}_{\bar{q}}^{\ell-*}(M / \mathcal{F})$ is non-degenerate for any two complementary perversities $\bar{p}$ and $\bar{q}$ ".

By using the Mayer-Vietoris technics of 3.6, 3.7 and Proposition 4.3.5 we reduce the problem to proving $\mathfrak{A}\left(\mathbb{R}^{n}, \mathcal{F}_{\mathbb{R}^{n}}\right)$, where $\left(\mathbb{R}^{n}, \mathcal{F}_{\mathbb{R}^{n}}\right) \equiv\left(c \mathbb{S}^{n-1}, c \mathcal{G}_{S}\right)$ is a slice of a tubular neighborhood of a singular stratum $S$ of $\mathrm{S}_{\mathcal{F}}$. In other words, we need to prove that the pairing

$$
P_{\mathbb{R}^{n}}: \mathbb{H}_{\bar{p}}^{*}\left(\mathbb{R}^{n} / \mathcal{F}_{\mathbb{R}^{n}}\right) \times \mathbb{H}_{\bar{q}, c}^{\ell_{\mathbb{R}^{n}}-*}\left(\mathbb{R}^{n} / \mathcal{F}_{\mathbb{R}^{n}}\right) \rightarrow \mathbb{R}
$$

is non-degenerate.

From Propositions 3.5.1, 3.5.2, 3.7.1 and 3.7.2 we have

$$
\mathbb{H}_{\bar{p}}^{i}\left(\mathbb{R}^{n} / \mathcal{F}_{\mathbb{R}^{n}}\right)= \begin{cases}\mathbb{H}_{\bar{p}}^{i}\left(\mathbb{S}^{n-1} / \mathcal{G}_{S}\right) & \text { if } i \leq \bar{p}(\vartheta) \\ 0 & \text { if } i \geq \bar{p}(\vartheta)+1\end{cases}
$$

and

$$
\mathbb{H}_{\bar{q}, c}^{\ell_{\mathbb{R}^{n}}-i}\left(\mathbb{R}^{n} / \mathcal{F}_{\mathbb{R}^{n}}\right)= \begin{cases}0 & \text { if } i \geq \ell_{\mathbb{R}^{n}}-\bar{q}(\{\vartheta\})-1, \\ \mathbb{H}_{\bar{q}}^{\ell_{\mathbb{R}^{n}}-i-1}\left(\mathbb{S}^{n-1} / \mathcal{G}_{S}\right) & \text { if } i \leq \ell_{\mathbb{R}^{n}}-\bar{q}(\{\vartheta\})-2 .\end{cases}
$$

Since $\bar{p}$ and $\bar{q}$ are complementary perversities on $\mathbb{R}^{n}$, we have

$$
\begin{aligned}
\bar{p}(\{\vartheta\})+\bar{q}(\{\vartheta\}) & =\bar{t}(\{\vartheta\})=n-\operatorname{dim} \mathcal{F}_{\mathbb{R}^{n}}-2=n-\operatorname{dim} \mathcal{G}_{S}-2 \\
& =\ell_{\mathbb{S}^{n-1}}-1=\ell_{\mathbb{R}^{n}}-2 .
\end{aligned}
$$

These formulæ give

$$
\mathbb{H}_{\bar{q}, \mathrm{c}}^{\ell_{\mathbb{R}^{n-i}}}\left(\mathbb{R}^{n} / \mathcal{F}_{\mathbb{R}^{n}}\right)= \begin{cases}0 & \text { if } i \geq \bar{p}(\{\vartheta\})+1, \\ \mathbb{H}_{\bar{q}}^{\ell_{\mathbb{S}^{n-1}-i}}\left(\mathbb{S}^{n-1} / \mathcal{G}_{S}\right) & \text { if } i \leq \bar{p}(\{\vartheta\}) .\end{cases}
$$

Now, an inductive argument on depth $S_{\mathcal{F}}$ yields $\mathfrak{A}\left(\mathbb{R}^{n}, \mathcal{F}_{\mathbb{R}^{n}}\right)$ from these three facts: 
(i) $\mathfrak{A}\left(\mathbb{S}^{n-1}, \mathcal{G}_{S}\right)$.

(ii) The pairing $P_{\mathbb{R}^{n}}$ becomes the pairing $P_{\mathbb{S}^{n-1}}$ through the isomorphism induced by (24) and (25).

Indeed, the operator $\aleph_{23}: \mathbb{H}_{\bar{p}}^{*}\left(\mathbb{S}^{n-1} / \mathcal{G}_{S}\right) \rightarrow \mathbb{H}_{\bar{p}}^{*}\left(c \mathbb{S}^{n-1} / c \mathcal{G}_{S}\right)$ defining $(24)$ is $\aleph_{23}([\alpha])=[\alpha]$; the operator $\aleph_{24}: \mathbb{H}_{\bar{q}}^{*}\left(\mathbb{S}^{n-1} / \mathcal{G}_{S}\right) \rightarrow \mathbb{H}_{\bar{q}, c}^{*}\left(c \mathbb{S}^{n-1} / \mathcal{G}_{S}\right)$ defining $(25)$ is $\aleph_{24}([\beta])=[g d t \wedge \beta]$. Since the action of $\Theta$ lives on $\mathbb{S}^{n-1}$, we can take a common tangent volume form $\eta$ for $\mathcal{F}_{\mathbb{R}^{n}}$ and $\mathcal{G}_{S}$ (cf. proof of Proposition 4.3.2). Now, for $[\alpha] \in \mathbb{H}_{\bar{p}}^{i}\left(\mathbb{S}^{n-1} / \mathcal{G}_{S}\right)$ and $[\beta] \in \mathbb{H}_{\bar{q}, c}^{\ell_{\mathbb{S}^{n-1}-i}}\left(\mathbb{S}^{n-1} / \mathcal{G}_{S}\right)$ we have

$$
\begin{aligned}
P_{\mathbb{R}^{n}}\left(\aleph_{23}[\alpha], \aleph_{24}[\beta]\right) & =\int_{\left.\mathbb{R}^{n} \times R_{\mathcal{F}_{S^{n-1}}} \times\right] 0,1[} \alpha \wedge g \wedge d t \wedge \beta \wedge \eta \\
& \left.=\int_{R_{\mathcal{F}_{S^{n-1}}}} \alpha \wedge \beta \wedge \eta\right)\left(\int_{0}^{1} g d t\right)=P_{\mathbb{S}^{n-1}}([\alpha],[\beta]) .
\end{aligned}
$$

(iii) The perversities $\bar{p}$ and $\bar{q}$ are complementary on $\mathbb{S}^{n-1}$.

Indeed, for any stratum $S \in \mathrm{S}_{\mathcal{G}}$,

$$
\begin{aligned}
\bar{p}(S)+\bar{q}(S) & =\bar{p}(S \times] 0,1[)+\bar{q}(S \times] 0,1[)=\bar{t}(S \times] 0,1[) \\
& =\operatorname{codim}_{\mathbb{R}^{n}} \mathcal{F}_{\mathbb{R}^{n}}-\operatorname{codim}_{S \times] 0,1[}\left(\mathcal{F}_{S} \times \mathcal{I}\right)-2 \\
& =\operatorname{codim}_{\mathbb{S}^{n-1}} \mathcal{G}_{S}-\operatorname{codim}_{S} \mathcal{F}_{S}-2=\bar{t}(S) .
\end{aligned}
$$

Hau amaia da.

\section{References}

[1] M. Bauer, Feuilletage singulier défini par une distribution presque régulière, Collect. Math. 37 (1986), 189-209.

[2] H. Boualem et P. Molino, Modèles locaux saturés de feuilletages riemanniens singuliers, C. R. Acad. Sci. Paris 316 (1993), 913-916.

[3] G. E. Bredon, Introduction to Compact Transformation Groups, Pure Appl. Math. 46, Academic Press, 1972.

[4] —, Topology and Geometry, Grad. Texts in Math. 139, Springer, 1993.

[5] J. L. Brylinsky, Equivariant intersection cohomology, in: Contemp. Math. 132, Amer. Math. Soc., 1992, 5-32.

[6] A. El Kacimi et G. Hector, Décomposition de Hodge basique pour un feuilletage riemannien, Ann. Inst. Fourier (Grenoble) 36 (1988), 207-227.

[7] A. El Kacimi, V. Sergiescu et G. Hector, La cohomologie basique d'un feuilletage riemannien est de dimension finie, Math. Z. 188 (1985), 593-599.

[8] M. Goresky and R. MacPherson, Intersection homology theory, Topology 19 (1980), $135-162$.

[9] S. Kobayashi, Transformation Groups in Differential Geometry, Springer, 1995.

[10] I. Kolař, P. W. Michor and J. Slovák, Natural Operations in Differential Geometry, Springer, 1993. 
[11] J. Kubarski, About Stefan's definition of a foliation with singularities: a reduction of axioms, Bull. Soc. Math. France 118 (1990), 391-394.

[12] P. Molino, Riemannian Foliations, Progr. Math. 73, Birkhäuser, 1988.

[13] J. I. Royo Prieto, Estudio cohomológico de flujos riemannianos, $\mathrm{PhD}$ thesis, Universidad del País Vasco, Euskal herriko Unibertsitatea, http://xtsunxet.usc.es/royoprieto/pdf/tesis.pdf.

[14] M. Saralegi, Homological properties of stratified spaces, Illinois J. Math. 38 (1994), $47-70$.

[15] M. Saralegi and R. Wolak, The BIC of a conical fibration, Math. Notes 77 (2005), 213-231.

[16] P. Stefan, Accessible sets, orbits, and foliations with singularities, Proc. London Math. Soc. 29 (1974), 699-713.

[17] H. J. Sussmann, Orbits of families of vector fields and integrability of distributions, Trans. Amer. Math. Soc. 180 (1973), 171-188.

[18] I. Vaisman, Lectures on the Geometry of Poisson Manifolds, Progr. Math. 118, Birkhäuser, 1994.

[19] A. Verona, Le théorème de de Rham pour les préstratifications abstraites, C. R. Acad. Sci. Paris 273 (1971), 886-889.

[20] V. P. Viflyantsev, Frobenius theorem for differential systems with singularities, Vestnik Moskov. Univ. 39 (1980), no. 3, 11-14 (in Russian).

[21] R. Wolak, Basic cohomology for singular riemannian foliations, Monatsh. Math. 128 (1999), 159-163.

Laboratoire de Mathématiques de Lens EA 2462

Institute of Mathematics

Fédération CNRS Nord-Pas-de-Calais FR 2956

Jagiellonian University

Reymonta 4

Faculté des Sciences Jean Perrin

Université d'Artois

Rue Jean Souvraz S.P. 18

30-059 Kraków, Poland

62307 Lens Cedex, France

E-mail: saralegi@euler.univ-artois.fr

Received 25.5.2005

and in final form 6.6.2006 